\title{
Extreme quasars at high redshift
}

\author{
M. L. Martínez-Aldama ${ }^{1}$, A. del Olmo ${ }^{1}$, P. Marziani ${ }^{2}$, J. W. Sulentic ${ }^{1}$, C. A. Negrete ${ }^{3}$, D. Dultzin ${ }^{4}$, \\ M. D'Onofrio ${ }^{5}$, and J. Perea ${ }^{1}$ \\ ${ }^{1}$ Instituto de Astrofisíca de Andalucía, IAA-CSIC, Glorieta de la Astronomía s/n, 18008 Granada, Spain \\ e-mail: maryloli@iaa.es \\ 2 Istituto Nazionale d'Astrofisica (INAF), Osservatorio Astronomico di Padova, 35122 Padova, Italy \\ 3 CONACYT Research Fellow, Instituto de Astronomía, UNAM, México DF 04510, Mexico \\ ${ }^{4}$ Instituto de Astronomía, UNAM, México DF 04510, Mexico \\ 5 Dipartimento di Fisica \& Astronomia "Galileo Galilei”, Università di Padova, Padova, Italy
}

Received 31 May 2018 / Accepted 24 July 2018

\begin{abstract}
Context. Quasars radiating at extreme Eddington ratios (hereafter xA quasars) are likely a prime mover of galactic evolution and have been hailed as potential distance indicators. Their properties are still scarcely known.

Aims. We aim to test the effectiveness of the selection criteria defined on the "4D Eigenvector 1" (4DE1) for identifying xA sources. We provide a quantitative description of their rest-frame UV spectra (1300-2200 $)$ in the redshift range $2 \lesssim z \lesssim 2.9$, with a focus on major emission features.

Methods. Nineteen extreme quasar candidates were identified using 4DE1 selection criteria applied to SDSS spectra: AlIII $\lambda 1860 /$ SiIII] $\lambda 1892 \gtrsim 0.5$ and CIII] $\lambda 1909 /$ SiIII] $\lambda 1892 \lesssim 1$. The emission line spectra was studied using multicomponent fits of deep spectroscopic observations $\left(S / N \gtrsim 40-50\right.$; spectral resolution $\left.\approx 250 \mathrm{~km} \mathrm{~s}^{-1}\right)$ obtained with the OSIRIS at Gran Telescopio Canarias (GTC).

Results. GTC spectra confirm that almost all of these quasars are xA sources with very similar properties. We provide spectrophotometric and line profile measurements for the SiIV $\lambda 1397+$ OIV, CIV $\lambda 1549+$ HeII $\lambda 1640$, and the $1900 \AA$ blend. This last feature is found to be predominantly composed of AlIII $\lambda 1860$, SiIII] $\lambda 1892$ and FeIII emission features, with weak CIII] $\lambda 1909$. The spectra can be characterized as very low ionization (ionization parameter, $\log U \sim-3$ ), a condition that explains the significant FeIII emission observed in the spectra. xA quasars show extreme properties in terms of CIV $\lambda 1549$ equivalent width and blueshift amplitudes. CIV $\lambda 1549$ shows low equivalent width, with a median value of $15 \AA$ ( $\leqslant 30 \AA$ for the most sources), and high or extreme blueshift amplitudes ($\left.5000 \lesssim c\left(\frac{1}{2}\right) \lesssim-1000 \mathrm{~km} \mathrm{~s}^{-1}\right)$. Weak-lined quasars appear as extreme xA quasars and not as an independent class. The CIV $\lambda 1549$ high amplitude blueshifts coexists in all cases save one with symmetric and narrower AlIII $\lambda 1860$ and SiIII] $\lambda 1892$ profiles. Estimates of the Eddington ratio using the AlIII $\lambda 1860 \mathrm{FWHM}$ as a virial broadening estimator are consistent with the ones of a previous xA sample. Conclusions. xA quasars show distinguishing properties that make them easily identifiable in large surveys and potential "standard candles" for cosmological applications. It is now feasible to assemble large samples of xA quasars from the latest data releases of the SDSS. We provide evidence that AlIII $\lambda 1860$ could be associated with a low-ionization virialized subsystem, supporting previous suggestions that AlIII is a reliable virial broadening estimator.
\end{abstract}

Key words. quasars: general - quasars: emission lines - quasars: supermassive black holes

\section{Introduction}

Quasars are found over an enormous range of distances $(z \sim$ $0-7.5)$ in the Universe. For this reason they have occasionally been cited as the ultimate possible standard candles for use in cosmology (see for example the Chapter by in D'Onofrio \& Burigana 2009 and the recent reviews by Sulentic et al. 2014b and Czerny et al. 2018). The problem with such a use has been the lack of a clear definition of "quasar" and a contextualization of their diversity. Since 2000 a clearer idea of their nature and diversity has emerged using the $4 \mathrm{D}$ Eigenvector 1 (4DE1) formalism. We are now able to identify a main sequence quasar (see Marziani et al. 2018, for a recent review), and recognize an extreme accretor (xA) quasar population at the end of this sequence (e.g., Marziani \& Sulentic 2014; hereafter MS14). This xA population radiating at $L / L_{\mathrm{Edd}} \sim 1$ offer the best opportunity to use quasars for cosmology (MS14, Wang et al. 2014). This paper describes a search for extreme quasars at $z \sim 2.3$ built upon an extension of low-redshift 4DE1 studies.

Quasar spectra show diverse properties in measures of line intensity ratios and line profiles. These measures offer multifold diagnostics of emitting region structure and physical conditions (Marziani et al. 2018). Organizing the diversity of quasar properties has been an ongoing effort for many years. Perhaps the first successful attempt was carried out by Boroson \& Green (1992). They proposed an Eigenvector 1 scheme based on a principal component analysis of the Palomar-Green quasar sample (see Gaskell et al. 1999; Sulentic et al. 2000a, for reviews up to the late 1990s). Trends between measures of [O III] $\lambda \lambda 44959,5007$, optical FeII emission and full width at half maximum (FWHM) $\mathrm{H} \beta$ were found, and appreciation of their importance has grown with time (Sulentic \& Marziani 2015, and references therein). Sulentic et al. (2000b) expanded upon this work and defined a new scheme called 4D Eigenvector 1 (4DE1) with the addition of two new parameters. Principal 4DE1 measures for low- $z$ 
quasars involve: (1) FWHM of broad line $\mathrm{H} \beta$ (excluding any narrow emission component) ${ }^{1}$; (2) the strength of the optical FeII blend at $4570 \AA$ normalized by the intensity of $\mathrm{H} \beta$ : $R_{\mathrm{FeII}}=\mathrm{I}(\mathrm{FeII}) / \mathrm{I}(\mathrm{H} \beta)$; (3) the velocity shift at half maximum $\left(c\left(\frac{1}{2}\right)\right)$ of the high-ionization line (HIL) CIV $\lambda 1549$ profile relative to a rest-frame (usually defined by measures of the [O III $] \lambda 5007$ and or narrow $\mathrm{H} \beta$ centroid) and (4) the soft X-ray photon index $\left(\Gamma_{\text {soft }}\right)$.

The 4DE1 optical plane (OP), defined by the measures of the FWHM of $\mathrm{H} \beta$ and $R_{\mathrm{FeII}}$, shows a reasonably welldefined sequence (a "main sequence" quasar, MS, Sulentic et al. 2000a; Marziani et al. 2001). The nomenclature is motivated by an analogy with the role of the stellar main sequence in the H-R diagram which connects observational measures to physical properties (Sulentic et al. 2008). The stellar MS is driven by stellar mass, while the quasar sequence is thought to be driven by Eddington ratio ( $\propto \dot{M}$, Marziani et al. 2001, 2003b). Sulentic et al. (2000a) noted a change in all 4DE1 measures near FWHM $\mathrm{H} \beta=4000 \mathrm{~km} \mathrm{~s}^{-1}$ in low- $z$ quasar samples $\left(L \lesssim 10^{47} \mathrm{erg} \mathrm{s}^{-1}\right)$. This change motivated an empirical designation of two quasar populations: Population A with FWHM H $\beta \leq 4000 \mathrm{~km} \mathrm{~s}^{-1}, R_{\mathrm{FeII}}>0.5$, frequent CIV $\lambda 1549$ profile blueshifts and sources with a soft X-ray excess, and Population B with FWHM $\mathrm{H} \beta>4000 \mathrm{~km} \mathrm{~s}^{-1}, R_{\text {FeII }}<0.5$, absence of CIV $\lambda 1549$ blueshift and little or no soft X-ray excess (Bensch et al. 2015). Radio-loud (RL) quasars are strongly concentrated in the Population B domain along with $30 \%$ of radio quiet (RQ) sources (e.g., Sulentic et al. 2003; Zamfir et al. 2008, and references therein).

Low-ionization emission lines (LILs $-\mathrm{H} \beta$ best studied feature) in quasars frequently show asymmetric profiles. The $\mathrm{H} \beta$ broad component $\left(\mathrm{H} \beta_{\mathrm{BC}}\right)$ in Population B sources is usually well described by a double Gaussian profile with one of the components centered on the rest-frame and the second one redshifted by $\approx 1000-3000 \mathrm{~km} \mathrm{~s}^{-1}$ (Zamfir et al. 2010), the very broad component (VBC). Population A sources rarely show the redshifted component. High-ionization line (CIV $\lambda 1549$ best studied prototype) profiles usually show blueward shifts/asymmetries in Population A sources. Pop. B objects also show weak or moderate strength LILs like FeII and the Ca II IR triplet (Sulentic et al. 2006b; Martínez-Aldama et al. 2015). Usually Population B quasars do not show any strong soft $\mathrm{X}$-ray excess (Sulentic et al. 2000a; Bensch et al. 2015, and references therein). Largely radio-quiet (RQ) Population A sources usually show symmetric profiles well-modeled with a Lorentz function (Sulentic et al 2002; Zamfir et al. 2010; Cracco et al. 2016). Population A sources with the narrowest $\mathrm{H} \beta$ profiles $\left(<2000 \mathrm{~km} \mathrm{~s}^{-1}\right)$ are often called narrow line Seyfert 1 sources (NLSy1), but in no sense represent a distinct class of quasars (Zamfir et al. 2008; Sulentic et al. 2015)

All of the above description involves quasars with $z<$ 1.0 where moderate to high $\mathrm{S} / \mathrm{N}$ ground-based spectra exist for significant numbers of sources $\mathrm{H} \beta$ (ground based) and CIV $\lambda 1549$ (HST FOS archival data). Within each quasar population systematic trends are revealed in composite spectra of $\mathrm{H} \beta$ (Sulentic et al. 2002; Zamfir et al. 2010) and CIV (Bachev et al. 2004). Sulentic et al. (2002) defined Pop. A subclasses A1, A2, $\mathrm{A} 3$ and $\mathrm{A} 4$ in order of increasing intervals of $0.5 R_{\mathrm{FeII}}$ have been defined. Extreme Population A sources in bins A3 and A4 (xA) involve quasars with very strong $R_{\mathrm{FeII}} \gtrsim 1.0$. This criterion is applicable only to sources at low redshift. This study involves

\footnotetext{
In the following we understand for broad profile of a line the total broad profile excluding the narrow component.
}

searching for higher redshift analogs of such extreme quasars. At high redshift $(z>2)$ the spectral range used to define sources in the 4DE1 (FWHM H $\beta, R_{\mathrm{FeII}}$ ) context are lost unless NIR spectra are available for $\mathrm{H} \beta$ (Marziani et al. 2009). Many CIV spectra in the intermediate and high- $z$ range are available in the SDSS and BOSS archives although low $\mathrm{S} / \mathrm{N}$ often precludes detailed analysis.

The 4DE1 provides a consistent picture of quasar observational properties in low $z$ samples: beginning with the low$R_{\mathrm{FeII}}$ and broad FWHM $\mathrm{H} \beta$, involving high black hole mass "disk-dominated" quasars. As we move along the sequence we encounter sources whose spectra show narrower LIL profiles, lower ionization spectra, and blueshifted CIV $\lambda 1549$ profiles providing evidence of strong HIL emitting outflows: "wind-dominated" quasars (Richards et al. 2011). Eddington ratio (convolved with the effects of orientation) appears to be the physical parameter driving systematic changes of observational properties along the quasar MS (Sulentic et al. 2000a; Marziani et al. 2001; Boroson 2002). The main sequence observational trends can be interpreted as driven by small $M_{\mathrm{BH}}$, higher $L / L_{\text {Edd }}$ (young? high accretors) toward the high $R_{\text {FeII }}$ end of the sequence. The $\mathrm{xA}$ sources cluster around $L / L_{\mathrm{Edd}} \approx 1^{2}$. This would imply that the low- $z$ xA sources are the "youngest" (less massive than Pop. B) quasar population radiating at the highest Eddington ratios (Fraix-Burnet et al. 2017).

There is a growing consensus that sources at the high $R_{\text {FeII }}$ end of the MS are accreting at the highest rates, and are expected to be close to the radiative limit per unit black hole mass (Sun \& Shen 2015; Du et al. 2016a,b; Sniegowska et al. 2018). If this is the case, $x A$ quasars acquire a special meaning. While Population A HILs are dominated by blue shifted emission associated with outflows, the presence of almost symmetric and unshifted LILs (Balmer lines, but also Paschen lines, La Franca et al. 2014) indicate the coexistence of a LIL emitting region that is virialized. Since $L / L_{\text {Edd }}$ tends toward a constant limiting value (Mineshige et al. 2000), xA quasars can be considered as "Eddington standard candles." If so that, if $M_{\mathrm{BH}}$ can be retrieved under the virial assumption, an estimate of the luminosity becomes possible since $L / L_{\mathrm{Edd}} \propto L / M_{\mathrm{BH}}$ (Wang et al. 2013; La Franca et al. 2014, MS14). This approach is conceptually analogous to the use of the link between the velocity dispersion in virialized systems (i.e., the rotational velocity of HI disks in spiral galaxies Tully \& Fisher 1977). Initial computations for samples of 100-200 low- $z$ quasars $(\lesssim 1)$ confirm the conceptual validity of the "virial luminosity" estimates (Negrete et al. 2017, 2018), although scatter in the distance modulus is still too large to draw meaningful inferences for cosmology.

4DE1 trends can also be helpful for interpreting high- $L$ and high- $z$ quasars, although there are two caveats. At high redshift, $z \sim 2$, the majority of sources show large FWHM due to a bias in luminosity (Sulentic et al. 2014a, 2017): quasars with luminosities comparable to the low $z$ low- $L$ sources are still too faint to be efficiently discovered. More fundamentally, there is a minimum possible FWHM $\mathrm{H} \beta$ at fixed luminosity, if the line emitting region is virialized and its size follows a scaling law with luminosity. In practice this means that at $\log L \gtrsim 47$, all lines have to be broader than FWHM $2000 \mathrm{~km} \mathrm{~s}^{-1}$. By the same token the FWHM limit for Population A becomes luminosity dependent. The limit established at $4000 \mathrm{~km} \mathrm{~s}^{-1}$ is valid only for low- $z$,

2 The precise $L / L_{\text {Edd }}$ values depend on the black hole mass $M_{\mathrm{BH}}$ scaling law and on the bolometric corrections. Following the 4DE1 based assumptions described above, the highest values along the MS are $L / L_{\text {Edd }} \approx 1-2$. 
relatively low- $L$ quasars. Sources with larger FWHM and emission line properties similar to the ones of the low- $z$ XA quasars have been found at high- $z$ and high- $L$ (Negrete et al. 2012, MS14). Another important issue at high- $L$ concerns the HIL blueshifts. While at low- $L$ large blueshifts $\left(v_{\mathrm{r}} \lesssim-1000 \mathrm{~km} \mathrm{~s}^{-1}\right)$ are confined to Pop. A (Sulentic et al. 2007; Richards et al. 2011), at high- $L$ they are ubiquitous (Coatman et al. 2016; Bischetti et al. 2017; Bisogni et al. 2017), even if Pop. A sources still show the largest blueshift amplitudes among all quasars (Sulentic et al. 2017; hereafter S17). At any rate, several recent studies confirm that $\mathrm{H} \beta$, observed in NIR spectra in quasars at $z \gtrsim 1$ shows fairly symmetric and unshifted profiles suggesting that the broadening is mainly due to virial motions of the line emitting gas (Marziani et al. 2009; Bisogni et al. 2017; Shen et al. 2016; Vietri et al. 2018, S17). We will show in this paper that this is probably true also for high- $L$ xA sources.

Goals of this paper include testing the effectiveness of 4DE1 selection criteria for identifying high $L / L_{\text {Edd }} \mathrm{xA}$ sources at $z \sim$ 2.4. This will enable us to analyze spectral properties of the identified $x A$ quasars in the rest-frame UV region. A sample of candidate $\mathrm{xA}$ sources (hereafter GTC-xA) was observed with the Gran Telescopio de Canarias (GTC) using the OSIRIS spectrograph. We apply the 4DE1 selection criterion defined by MS14 using UV diagnostic ratios: AlIII $\lambda 1860 /$ SiIII] $\lambda 1892 \gtrsim 0.5$ and CIII] $\lambda 1909 /$ SiIII] $\lambda 1892 \lesssim 1.0$. The selected sources are intended to represent an xA population for which $R_{\mathrm{FeII}}$ is expected to be larger than 1, with no limitation on line FWHM. The sample selection is described in Sect. 2. The observations and the data reduction are presented in Sect. 3. We perform a multicomponent fitting and build Monte Carlo (MC) simulations to estimate measurement uncertainties (Sect. 4 and Appendix B). Spectra and line measures are presented for the $1900 \AA$ blend, the blend CIV $\lambda 1549+$ HeII $\lambda 1640$, and the SiIV $\lambda 1397$ region in Sect. 5. A comparison with control samples at low- $z$ and or $L$ is described in the Sect. 6.1. A composite spectrum for the GTC-xA sample (Sect. 5.1) allows us to carry out a comparison with low- $z$ and low- $L$ samples (Sect. 6.2). We discuss the low-ionization spectra and identify FeIII and FeII features that are prominent in our spectra (Sects. 6.3 and 6.4). After estimating the main accretion parameters (Sect. 6.5), we briefly analyze the relation of xA sources to Weak Line Quasars (WLQs) - a related class of quasars with extreme properties (Sect. 6.6).

xA sources are especially important because they are the quasars radiating at the highest luminosity per unit mass. The extreme radiative properties of $x$ As make them prime candidates for maximum feedback effects on host galaxies. We briefly analyze the possibility of significant feedback effects in Sect. 6.7. We conclude the paper with some consideration on the possible cosmological exploitation of xA quasars at high-z (Sect. 7).

\section{Sample description}

\subsection{GTC-XA sample}

MS14 extracted 3000 quasar spectra from the SDSS DR6 archive which provided coverage of the $1900 \AA$ blend for sources in the redshift range $2.0<z<2.9$, with $g<19.5$. Intensity measures of AlIII 11860 , SiIII] $\lambda 1892$ and CIII] $\lambda 1909$ were carried out with an automatic SPLOT procedure within the IRAF reduction package. The majority of SDSS spectra are quite noisy making them suitable for identifying samples of candidate sources, but not providing accurate spectroscopic measures (Sulentic \& Marziani 2015). A preliminary selection of extreme Eddington candidates was made and sources were then vetted according to the $\mathrm{xA}$ selection criteria (AlIII $\lambda 1860 /$ SiIII] $\lambda 1892 \gtrsim 0.5$ and CIII] $\lambda 1909 /$ SiIII] $\lambda 1892 \lesssim 1.0$ ). MS14 considered the brightest candidates with moderate $\mathrm{S} / \mathrm{N}$ $(\geq 15)$ spectra, leaving 58 candidate $\mathrm{xA}$ quasars whose relative AlIII $\lambda 1860$, SiIII] $\lambda 1892$ and CIII] $\lambda 1909$ intensities satisfied the selection criteria, but whose $\mathrm{S} / \mathrm{N}$ was too poor to make an accurate measurement of individual lines in the $1900 \AA$ blend. Hence the need for new GTC spectroscopic observations. This paper present an analysis of 19 of the sources that constitute our GTCxA sample.

Table 1 gives source identifications and basic properties including: redshift and uncertainty (Cols. 2 and 3), emission line used for the redshift estimate (Col. 4), apparent $V$ magnitude and absolute $B$ magnitude $M_{\mathrm{B}}$ (Cols. 5 and 6) as given in Véron-Cetty \& Véron (2010), and $(g-r)$ color index from the SDSS photometry (Col. 7). Column 8 identifies other observed features like its classification as BAL (Broad Absorption Line) or mini-BAL quasar, and the radio properties, if they are detected. Low- $z$ studies (Zamfir et al. 2008) define a radio-loud (RL) quasar with a radio/optical flux ratio $\log R_{\mathrm{K}} \gtrsim 1.8$ (Kellermann et al. 1989), or better a radio power measure $\log P_{v}>31.6\left[\mathrm{erg} \mathrm{s}^{-1} \mathrm{~Hz}^{1}\right.$ ], independent of uncertainties in optical flux measures. $R_{\mathrm{K}}$ was obtained normalizing the $k$-corrected radio flux at $1.4 \mathrm{GHz}$ by the $k$-corrected $B$ magnitude. Two radio-detected sources exceed the $R_{\mathrm{K}}$ limit with the third one close to the limit. Only for SDSSJ233132.83+010620.9 was possible to estimate the radio spectral index from one observation at $8.4 \mathrm{GHz}$ (Cegłowski et al. 2015): $\alpha_{\mathrm{r}} \approx 0.44$, that places it in the compact steep-spectrum radio domain. SDSSJ234657.25+145736.0 has only a lowresolution NRAO VLA Sky Survey (NVSS) map available. In this case, $P_{v}$ and $R_{\mathrm{K}}$ have been computed assuming $\alpha_{\mathrm{r}}=0$. All of them exceed the $\log P_{v}$ limit, then we could have three RL quasars in GTC-xA sample. Since low- $z$ RL are almost never found in the $\mathrm{xA}$ domain in $4 \mathrm{DE} 1$, it is possible either that the three radio detected sources are not xA extreme accretors or that high- $z$ Pop. A quasars are more frequently RL. At this point the three RL sources in the sample must be treated with caution.

\subsection{The FOS-A, S14-A, and FOS-xA "control" samples}

The quasars in the GTC-xA sample are thought to be highly accreting, with an average bolometric luminosity of $\log L \sim$ $47\left[\mathrm{erg} \mathrm{s}^{-1}\right]$. Absolute magnitude $M_{\mathrm{B}} \approx-26$, before extinction correction, corresponds to a comoving space density of $\sim 10^{-6} \mathrm{mag}^{-1} \mathrm{Mpc}^{-3}$ just beyond the turnover at the high luminosity end of the $2 \mathrm{dF}$ luminosity function (Boyle et al. 2000).

In order to compare the behavior of the GTC-xA sample, we consider the FOS sample from Sulentic et al. (2007) as a control sample at low- $L$ and low- $z$. For the sake of the present paper, we restrict the control FOS sample to 28 Pop. A RQ sources covering the CIV and $1900 \AA$ blend spectral range and with previous measures for the $\mathrm{H} \beta$ profile and $R_{\text {FeII }}$ (Marziani et al. 2003a). 23 objects are classified as Pop. A1-A2 sources (henceforth FOSA sample) and five as $\mathrm{xA}$ sources (hereafter FOS-xA sample) including I Zw 1 . The FOS sample has a typical bolometric luminosity $\log L \sim 45.2\left[\mathrm{erg} \mathrm{s}^{-1}\right.$ ] and a redshift $z \lesssim 0.5$.

The sample presented in Sulentic et al. (2014a, hereafter S14) covers a similar range in redshift $(z \sim 2.3$, corresponding to a lookback time of $\approx 10 \mathrm{Gyr}$ ), and are in turn a factor approximately ten less luminous $(\log L \sim 46)$ than the GTC-xA sample. S14 is representative of a general population of faint, moderately accreting quasars that are also found at intermediate 
Table 1. Source identification and basic properties of the GTC-xA quasars.

\begin{tabular}{|c|c|c|c|c|c|c|c|}
\hline $\begin{array}{l}\text { SDSS identification } \\
\text { (1) }\end{array}$ & $\begin{array}{c}z \\
(2)\end{array}$ & $\begin{array}{l}\Delta z \\
(3)\end{array}$ & $\begin{array}{c}\text { Line } \\
(4)\end{array}$ & $\begin{array}{l}m_{v} \\
(5)\end{array}$ & $\begin{array}{c}M_{\mathrm{B}} \\
(6) \\
\end{array}$ & $\begin{array}{c}g-r \\
(7)\end{array}$ & $\begin{array}{c}\text { Comments } \\
(8)\end{array}$ \\
\hline SDSSJ000807.27-103942.7 & 2.4660 & 0.0010 & III & 19.15 & -26.2 & 0.08 & \\
\hline SDSSJ004241.95+002213.9 & 2.0560 & 0.0053 & III & 19.05 & -25.6 & 0.24 & \\
\hline SDSSJ021606.41+011509.5 & 2.2236 & 0.0008 & $\mathrm{I}, \mathrm{II}$ & 19.36 & -25.2 & 0.49 & BAL \\
\hline SDSSJ024154.42-004757.5 & 2.3919 & 0.0015 & III & 19.24 & -26.0 & 0.24 & \\
\hline SDSSJ084036.16+235524.7 & 2.1879 & 0.0012 & III & 19.46 & -25.3 & 0.40 & \\
\hline SDSSJ101822.96+203558.6 & 2.2502 & 0.0035 & III & 19.27 & -25.5 & 0.33 & \\
\hline SDSSJ103527.40+445435.6 & 2.2639 & 0.0060 & III & 19.34 & -25.6 & 0.20 & \\
\hline SDSSJ105806.16+600826.9 & 2.9406 & 0.0001 & $\mathrm{I}, \mathrm{II}$ & 19.29 & -26.7 & 0.16 & \\
\hline SDSSJ110022.53+484012.6 & 2.0884 & 0.0028 & III & 18.90 & -25.9 & 0.13 & \\
\hline SDSSJ125659.79-033813.8 & 2.9801 & 0.0004 & II & 19.27 & -26.7 & 0.26 & BAL \\
\hline SDSSJ131132.92+052751.2 & 2.1234 & 0.0009 & III & 19.06 & -25.4 & 0.72 & BAL \\
\hline SDSSJ143525.31+400112.2 & 2.2615 & 0.0006 & III & 18.30 & -26.6 & 0.17 & \\
\hline SDSSJ144412.37+582636.9 & 2.3455 & 0.0018 & III & 19.21 & -25.8 & 0.37 & Mini-BAL \\
\hline SDSSJ151258.36+352533.2 & 2.2382 & 0.0012 & III & 19.21 & -25.8 & 0.04 & $\mathrm{RL}, \log P_{v} \approx 33.8, \log R_{\mathrm{K}} \approx 2.65$ \\
\hline SDSSJ214009.01-064403.9 & 2.0808 & 0.0038 & III & 19.26 & -25.3 & 0.56 & BAL \\
\hline SDSSJ220119.62-083911.6 & 2.1840 & 0.0015 & III & 18.68 & -26.1 & 0.35 & BAL \\
\hline SDSSJ222753.07-092951.7 & 2.1639 & 0.0010 & III & 19.11 & -25.6 & 0.10 & \\
\hline SDSSJ233132.83+010620.9 & 2.6271 & 0.0038 & III & 19.19 & -26.1 & 0.33 & $\mathrm{RL}, \log P_{v} \approx 34.1, \log R_{\mathrm{K}} \approx 2.93$ \\
\hline SDSSJ234657.25+145736.0 & 2.1682 & 0.0007 & III & 19.11 & -25.7 & 0.29 & $\mathrm{RI}, \log P_{v} \approx 32.7, \log R_{\mathrm{K}} \approx 1.67$ \\
\hline
\end{tabular}

Notes. Columns are as follows: (1) SDSS coordinate name. (2) Redshift. (3) Redshift uncertainty. (4) Lines used for redshift determination: I: CII $\lambda 1335$, II: OI+SiII $\lambda 1305$, III: AlIII $\lambda 1860+$ SiIII] $\lambda 1892$. (5) Apparent Johnson $V$ magnitude as reported by Véron-Cetty \& Véron (2010). (6) Absolute $B$ magnitude according to Véron-Cetty \& Véron (2010). (7) $g-r$ color in magnitudes. (8) Quasar classification: BAL QSO: Broad Absorption Line Quasar; Mini-BAL: Mini Broad Absorption Line Quasar, radio-loud (RL) and radio-intermediate (RI). $P_{v}$ is power per unit frequency at $1.4 \mathrm{GHz}$ in units of $\mathrm{erg} \mathrm{s}^{-1} \mathrm{~Hz}^{-1}$.

redshift (Fraix-Burnet et al. 2017). S14 includes both Pop. A and B sources, but no xA sources. Restriction to the eleven Pop. A quasars (hereafter S14-A) offers a high-z counterpart to the FOS-A sample, and therefore suitable for a comparison between xAs and a sample of Pop. A quasars of moderate $L$ at $z \sim 2-2.5$, that represents a population expected to be relatively common $\left(\Phi \sim 10^{-6} \mathrm{mag}^{-1} \mathrm{Mpc}^{-3}\right)$.

\section{Observations, data reduction and extinction estimation}

\subsection{Observations and data reduction}

Long slit observations were carried out in service mode using the OSIRIS spectrograph at the $10.4 \mathrm{~m}$ GTC telescope of the Roque de los Muchachos Observatory. Grisms R1000B and R1000R with $2 \times 2$ CCD binning were used for the observations, depending on the redshift of the source. The majority of the observations employed $\mathrm{R} 1000 \mathrm{~B}$ that covered the wavelength range from 3650-7400 ^ with a reciprocal dispersion of $2.1 \AA$ per pixel $(R \approx$ 1000). In our highest $z$ sources, SDSS J105806.16+600826.9, SDSS J125659.79-033813.8, and SDSS J233132.83+010620.9 with $z \approx 2.94,2.98$ and 2.63, respectively, we used the R1000R grism with reciprocal dispersion of $2.6 \AA \mathrm{pixel}^{-1}$ and spectral coverage 5100-10000 $\AA$. These two spectral ranges correspond the rest-frame region covering the UV spectral features of interest such as the SiIV $\lambda 1397$, CIV $\lambda 1549$ and the $1900 \AA$ blend. The spectra were obtained with an 0.6 arcsec slit width oriented at the parallactic angle in order to minimize atmospheric differential refraction. Table 2 contains a summary of the observations including: SDSS identification, observation date, grism employed, total exposure time for the three individual exposures
Table 2. Log of observations.

\begin{tabular}{cccccc}
\hline \hline $\begin{array}{c}\text { SDSS identification } \\
(1)\end{array}$ & $\begin{array}{c}\text { Obs. date } \\
(2)\end{array}$ & $\begin{array}{c}\text { Grism } \\
(3)\end{array}$ & $\begin{array}{c}\text { Exp. time } \\
(4)\end{array}$ & $\begin{array}{c}\text { Seeing } \\
(5)\end{array}$ & $\begin{array}{c}S / N \\
(6)\end{array}$ \\
\hline SDSSJ000807.27-103942.7 & $22 / 07 / 2015$ & $\mathrm{R} 1000 \mathrm{~B}$ & 1800 & 1.13 & 29 \\
SDSSJ004241.95+002213.9 & $22 / 07 / 2015$ & $\mathrm{R} 1000 \mathrm{~B}$ & 1440 & 1.18 & 39 \\
SDSSJ021606.41+011509.5 & $14 / 08 / 2015$ & $\mathrm{R} 1000 \mathrm{~B}$ & 1440 & 0.80 & 70 \\
SDSSJ024154.42-004757.5 & $14 / 08 / 2015$ & $\mathrm{R} 1000 \mathrm{~B}$ & 2700 & 1.17 & 40 \\
SDSSJ084036.16+235524.7 & $18 / 04 / 2015$ & $\mathrm{R} 1000 \mathrm{~B}$ & 1800 & 1.13 & 31 \\
SDSSJ101822.96+203558.6 & $12 / 06 / 2015$ & $\mathrm{R} 1000 \mathrm{~B}$ & 1440 & 1.40 & 33 \\
SDSSJ103527.40+445435.6 & $23 / 03 / 2015$ & $\mathrm{R} 1000 \mathrm{~B}$ & 1800 & 1.33 & 32 \\
SDSSJ105806.16+600826.9 & $12 / 06 / 2015$ & $\mathrm{R} 1000 \mathrm{R}$ & 2700 & 1.22 & 22 \\
SDSSJ110022.53+484012.6 & $18 / 04 / 2015$ & $\mathrm{R} 1000 \mathrm{~B}$ & 1890 & 0.74 & 38 \\
SDSSJ125659.79-033813.8 & $23 / 05 / 2015$ & $\mathrm{R} 1000 \mathrm{R}$ & 1440 & 1.29 & 12 \\
SDSSJ131132.92+052751.2 & $23 / 05 / 2015$ & $\mathrm{R} 1000 \mathrm{~B}$ & 1800 & 1.42 & 31 \\
SDSSJ143525.31+400112.2 & $11 / 04 / 2015$ & $\mathrm{R} 1000 \mathrm{~B}$ & 2340 & 0.98 & 60 \\
SDSSJ144412.37+582636.9 & $25 / 06 / 2015$ & $\mathrm{R} 1000 \mathrm{~B}$ & 1980 & 0.89 & 23 \\
SDSSJ151258.36+352533.2 & $11 / 04 / 2015$ & $\mathrm{R} 1000 \mathrm{~B}$ & 2340 & 0.86 & 37 \\
SDSSJ214009.01-064403.9 & $05 / 08 / 2015$ & $\mathrm{R} 1000 \mathrm{~B}$ & 1800 & 1.13 & 60 \\
SDSSJ220119.62-083911.6 & $17 / 06 / 2015$ & $\mathrm{R} 1000 \mathrm{~B}$ & 2340 & 0.83 & 75 \\
SDSSJ222753.07-092951.7 & $26 / 06 / 2015$ & $\mathrm{R} 1000 \mathrm{~B}$ & 1800 & 0.91 & 62 \\
SDSSJ233132.83+010620.9 & $05 / 08 / 2015$ & $\mathrm{R} 1000 \mathrm{R}$ & 1800 & 1.29 & 22 \\
SDSSJ234657.25+145736.0 & $16 / 07 / 2015$ & $\mathrm{R} 1000 \mathrm{~B}$ & 1440 & 0.96 & 54 \\
\hline
\end{tabular}

Notes. Columns are as follows: (1) SDSS identification. (2) Observation Date. (3) Grism. (4) Exposure time in seconds. (5) Seeing in arcseconds. (6) $\mathrm{S} / \mathrm{N}$ measured in the continuum at $1450 \AA$.

on each source, seeing estimated from the FWHM of field stars in the acquisition image, and the estimated $\mathrm{S} / \mathrm{N}$ in the $1450 \AA$ continuum region on the blue side of CIV $\lambda 1549$.

Data reduction was carried out in a standard way using the IRAF package. Bias subtraction and flat-fielding correction were performed nightly. Wavelength calibration was obtained using $\mathrm{Hg}+\mathrm{Ar}$ and $\mathrm{Ne}$ lamps observed with the same configuration and slit width used for source observations. Wavelength calibration rms was less than $0.1 \AA$. We checked the wavelength calibration for individual exposures with sky lines before source extraction, 
background substraction, and final combination. Scatter of the sky line wavelength peaks was $\lesssim 20 \mathrm{~km} \mathrm{~s}^{-1}$. This value provides a realistic estimate of the wavelength scale uncertainty including zero point error. Spectral resolution estimated from FWHM of the skylines is $\sim 230 \mathrm{~km} \mathrm{~s}^{-1}$ and $250 \mathrm{~km} \mathrm{~s}^{-1}$ for grisms R1000B and R1000R, respectively.

Instrumental response and flux calibration were obtained nightly with observations of the spectrophotometric standard stars Ross 640, GD24-9, Feige 110, Hiltner 600, and G158-100. In order to improve flux calibration, we also included two additional flux standard stars, LDS749B and HZ21, as target objects. They were observed with both grisms and two slits: 0.6 arcsec b (as used for quasar observations) and 5 arcsec. A comparison between the different slits gives a change in the absolute flux calibration $\sim 10 \%$. Spectra were corrected for light losses due to the narrow slit width employed and also to differential light loss as a function of wavelength. Taking into account the ratio between the slit width and the seeing during an observation allowed correction for the scale factor and for the wavelength dependence of the seeing following the method described by Bellazzini (2007). Telluric absorptions, that affect our spectra mainly beyond $7600 \AA$, were also corrected using the standard stars to obtain a normalized template of the absorption features. The template was shifted if needed, and scaled for each individual source in an iterative and interactive procedure, until the residuals in the telluric correction were negligible. Spectra were finally deredshifted as explained in Sect. 4.1.

\subsection{Extinction estimation}

It is visually apparent from examining our spectra that some of them (e.g., SDSSJ021606.41+011509.5, Fig. A.3) show a flatter continuum than cannot be modeled with a single power-law over the observed spectral range. This effect, as well as the presence of BALs, has been interpreted in the literature as indicating the presence of dust or internal reddening. In order to assess the importance of internal reddening on the observed fluxes and derived magnitudes in our XA sample, we have estimated the reddening in each source by fitting its UV continuum with quasar templates excluding spectral regions with broad emission lines (e.g., Ly $\alpha$, Sirv $\lambda 1397$, CIV $\lambda 1549$ and the $1900 \AA$ blend). We used four QSO templates: (1) a median composite spectrum representative of the XA quasar population and built with extreme accretor sources identified in the SDSS DataBase by MS14 excluding BALs; (2) a template involving the composite FIRST Bright Quasar Survey spectrum (FBQS; Brotherton et al. 2001); (3) the composite spectrum provided by Harris et al. (2016) with BOSS spectra of quasars in the redshift range $2.1<z<3.5$, and (4) the SDSS composite quasar spectra (Vanden Berk et al. 2001). We reddened the templates using an SMC extinction law (Gordon \& Clayton 1998) which appears to be the most appropriate reddening law for modeling quasar spectra (York et al. 2006; Gallerani et al. 2010). We assumed a $R_{\mathrm{V}}$ coefficient of 3.07 for the extinction law.

In general, best fits were obtained with the xA composite although there were no appreciable differences and all the fittings yielded similar results. For the majority of sources (twelve) no additional extinction was needed and the continuum was well represented by the templates. In six cases the reddening has a significant effect on the spectrum, amounting to $A_{\mathrm{V}}=0.1$ to $A_{\mathrm{V}}=0.32$. Table 3 reports reddening estimates parametrized by the $A_{V}$ value. In the case of SDSSJ220119.62-083911.6, classified as BAL, the spectrum shows a broad and deep absorption in the blue wings of CIV $\lambda 1549$, SiIV $\lambda 1397$ and Ly $\alpha$ with an evident
Table 3. Extinction measures for the xA sources.

\begin{tabular}{cc}
\hline \hline SDSS identification & $A_{\mathrm{V}}(\mathrm{mag})$ \\
\hline SDSSJ021606.41+011509.5 & 0.270 \\
SDSSJ103527.40+445435.6 & 0.178 \\
SDSSJ131132.92+052751.2 & 0.325 \\
SDSSJ144412.37+582636.9 & 0.106 \\
SDSSJ214009.01-064403.9 & 0.250 \\
SDSSJ233132.83+010620.9 & 0.150 \\
\hline
\end{tabular}

flattening of the continuum at wavelengths shorter than $1600 \AA$. However, from $1700 \AA$ the spectrum shows a similar slope to the templates and does not show evidence for reddening. Hence no internal reddening was applied to this source and the continuum at $1350 \AA$ needed for estimation of the luminosity was obtained by extrapolating the power law of the fit applied to the red spectral region.

\section{Data analysis}

\subsection{Redshift determination}

Accurate redshift estimates are very important because redshift defines the quasar rest-frame from which emission line shifts can be measured. Shifts are particularly important for HILs like CIV $\lambda 1549$ (e.g., Gaskell 1982; Espey et al. 1989; Carswell et al. 1991; Marziani et al. 1996). Redshift estimates are chiefly obtained from narrow emission lines (or the narrow core of $\mathrm{H} \beta$ ) for low- $z$ sources (e.g., Eracleous \& Halpern 2003; $\mathrm{Hu}$ et al. 2008). In the UV region covered by our spectra of $z \approx 2.3$, narrow LILs are not present. We must resort to broad LILs and estimate the redshifts using three features: AlIII $\lambda 1860$, CII $\lambda 1335$ and OI $\lambda 1304+$ SiII $\lambda 1306$. The strongest and hence most often detected LIL involves AlIII $\lambda 1860$, which emerged in lower $z$ studies as a kind of UV surrogate $\mathrm{H} \beta$ (Bachev et al. 2004). While often detected, it is always part of the $1900 \AA$ blend albeit on the blue end of it. Although AlIII $\lambda 1860$ and SiIII] $\lambda 1892$ are blended lines, they are stronger than CII $\lambda 1335$ and OI $\lambda 1304+$ SiII $\lambda 1306$ and in many of the spectra their peaks are clearly seen. We performed a multicomponent fit for each source, considering all the lines in the region of the $1900 \AA$ blend (see Sect. 4.2). The peaks of AlIII $\lambda 1860$ and SiIII] $\lambda 1892$ were unconstrained in intensity in the fitting and the adopted model of the blend was the one with minimum $\chi^{2}$. CII $\lambda 1335$ is the only isolated LIL in the observed UV range and is detected in only five sources (e.g., SDSSJ021606.41+011509.5, see Fig. A.3). The OI $\lambda 1304+\operatorname{SiII} \lambda 1306$ blend is also well seen in a few sources where CII $\lambda 1335$ is detected. In the rest of the sample these lines are too weak to be useful and are often affected by absorption features.

We constructed synthetic Gaussian profiles for CII $\lambda 1335$ and OI $\lambda 1304+$ SiII $\lambda 1306$ lines using the IRAF task MK1DSPEC assuming $F W H M \sim 4000 \mathrm{~km} \mathrm{~s}^{-1}$. We computed a redshift from the peak of the synthetic features. When the AlIII $\lambda 1860$ redshift was compared with the one of CII $\lambda 1335$ and OI $\lambda 1304+\operatorname{SiII} \lambda 1306$, we find three cases where the difference is less than $100 \mathrm{~km} \mathrm{~s}^{-1}$. In these cases, we kept the redshift value determined by the CII $\lambda 1335$ and OI $\lambda 1304+$ SiII $\lambda 1306$ (label I and II in Table 1). In the cases where the difference is larger than $100 \mathrm{~km} \mathrm{~s}^{-1}$, we considered the redshift given by AlIII $\lambda 1860$ and SiIII] $\lambda 1892$ (label III in Table 1), since the peak of these lines is clearly observed. The uncertainty reported in Col. 4 of Table 1 has been 
computed from the redshift difference between OI $\lambda 1304+$ SiII $\lambda 1306$, and AlIII $\lambda 1860+$ SiIII] $\lambda 1892$.

\subsection{Multicomponent fitting}

Emission lines can be distinguished by their ionization potential (IP). The UV range covered in our spectra is populated by intermediate (IP $\sim 20-40 \mathrm{eV}$ ) and high ionization lines (IP $>40 \mathrm{eV}$ ). They offer an opportunity to characterize the behavior of different ionic species at the same time. In order to analyze the emission lines in our spectra, we carried out multicomponent fits using the SPECFIT routine from IRAF (Kriss 1994). This routine simultaneously fits the continuum, and emission/absorption line components. The best model is indicated by the minimum $\chi^{2}$ over a spectral range where all components are included.

The main continuum source in the UV region is thought to arise from the accretion disk (Malkan \& Sargent 1982). In the absence of extinction the continuum can be modeled by a single power-law over the full observed spectral range. However, due to the presence of absorptions (BAL sources) or dust extinction the continuum is flattened out in several sources (Sect. 3.2). Whenever possible, we fit the entire spectra range with a single power-law or a linear continuum. Otherwise we estimate locally the continuum. We divide the observed spectral range in three parts, which are centered on the most important emission lines relevant to our work.

REGION 1: 1700-2200 $\AA$. This range is dominated by the $1900 \AA$ blend which includes AlIII $\lambda 1860$, SiII] $\lambda 1892$, CIII] $\lambda 1909$ and FeIII lines (see Sect. 5.6 and Appendix B for FeIII line identification). On the blue side of the blend SiII $\lambda 1816$ and NIII] $\lambda 1750$ are also detected. AlIII, SiIII] and CIII] are intermediate-ionization lines (IIL) and according to Negrete et al. (2012; hereafter N12) can be well-modeled with Lorentzian profiles. The strengths of the three lines are allowed to vary freely in our SPECFIT model. FWHM AlIII and SiIII] were assumed equal, while FWHM CIII] was unconstrained. SiII $\lambda 1816$ and NIII] $\lambda 1750$ were also modeled with Lorentzian profiles with flux and FWHM allowed to vary freely. All Lorentzian profile peaks were fixed at rest-frame. FeII makes an important contribution in the range 1715-1785 $\AA$. We tried to use templates available in the literature (Brühweiler \& Verner 2008; Mejía-Restrepo et al. 2016), but we could not reproduce the observed contribution. If that template is scaled to reproduce these features, the FeII emission around the MgII $\lambda 2800$ line would be overestimated by a large factor. On the converse if the template is normalized to FeII in the proximity of $\mathrm{MgII} \lambda 2800$, the FeII emission in the spectral region $1700-2200 \AA$ is negligible. We therefore decided to fit isolated Gaussian profiles for FeII at 1715 and $1785 \AA$. Their flux and FWHM vary freely. FeIII emission makes a larger contribution than FeII and appears especially strong on the red side of the $1900 \AA$ blend. We modeled the emission of this ion with the Vestergaard \& Wilkes (2001) template and included an extra component at $1914 \AA$ following N12 (the motivation for this choice is discussed in Sect. 6.3). Around 2020-2080 ^ we were forced to include extra Gaussians in order to obtain a good fit (we found excess emission with respect to the FeIII template). The flux and FWHM of the features at 2020 $2080 \AA$ were allowed also to vary freely.

REGION 2: 1450-1700 ̊. The CIV $\lambda 1549$ emission line dominates this region and is accompanied by HeII $\lambda 1640$, OIII] $\lambda 1663$ and AlII $\lambda 1670$. The broad component (BC) of CIV is modeled by a Lorentzian profile fixed at the rest-frame. The flux of the $\mathrm{CIV}_{\mathrm{BC}}$ is free and FWHM is assumed to be the same as AlIII $\lambda 1860$ and SiIII] 11892 . All the CIV profiles in our sample show a blueshift and or blueward asymmetry. In order to model it with SPECFIT, we used one or two blueshifted skewed Gaussian profiles. The flux, FWHM, asymmetry and shift were unconstrained. HeII $\lambda 1640$ was modeled assuming components similar to those of CIV $\lambda 1549$ : Lorentzian and skewed Gaussian profiles for the $\mathrm{BC}$ and blueshifted components, respectively. The FWHM, shift and asymmetry were assumed equal to those of CIV $\lambda 1549$, but the flux varies freely. OIII] $\lambda 1663$ and AlII $\lambda 1670$ were also modeled with unshifted Lorentzian profiles with their fluxed and FWHM varying freely.

REGION 3: 1300-1450 $\mathrm{A}$. The dominant emission in this region involves SiIV $\lambda 1397+$ OIV (the $1400 \AA$ blend), and is accompanied by weaker SiII $\lambda 1306$, OI $\lambda 1304$ and CII $\lambda 1335$ lines. The underlying assumption of the blend modeling is that the $\mathrm{BC}$ emission is dominated by SiIV $\lambda 1397$ due to collisional deexcitation of the inter combination OIV multiplet (Wills \& Netzer 1979), while the blue shifted component is due to an inextricable contribution of both OIV+SiIV $\lambda 1397$. The SiIV $\lambda 1397+$ OIV feature is a high-ionization blend, and shows a blueshifted, asymmetric profile not unlike Civ $\lambda 1549$. The broad component was modeled with the same emission components of CIV, whenever possible (in several cases the blue side of the SiIV $\lambda 1397+$ OIV blend was strongly contaminated by absorption features making a good fit impossible), with only the flux varying freely. In the case of strong absorption the blueshifted emission was modeled independently from the one of CIV.

For lines that are composed of more than one component (CIV $\lambda 1549$, HeII $\lambda 1640$ and SiIV $\lambda 1397+$ OIV) the total profile parameters were also computed: FWHM, centroid at half maximum $\left(c\left(\frac{1}{2}\right)\right)$, and asymmetry index (AI) defined by Zamfir et al. (2010). Unlike the SPECFIT components, these parameters provide a description of the profile that is not dependent on the model decomposition of the profile.

Figure 1 shows the multicomponent fitting made to the composite spectrum obtained by combining the normalized spectra of the GTC-xA sample (see Sect. 5.1 for a complete description). The bottom panels of the figure present the fits performed on the SiIV $\lambda 1397$, CIV $\lambda 1549$ and AlIII $\lambda 1860$ spectral regions of the continuum subtracted spectrum. Residuals of the fits are shown in the lower part of the bottom panels. Spectra and multicomponent fits for the individual 19 quasars analyzed in this paper are shown in Figs. A.1-A.19. Error estimations of blended emission lines were evaluated by building Monte-Carlo (MC) simulations as explained in Appendix B.

\section{Results}

\subsection{Composite spectrum}

We constructed a median composite spectrum from the individual observations in order to emphasize the main emission features (composites efficiently remove narrow absorption lines) and carry out a comparison with other samples (see Sect. 6.2). The composite GTC-xA spectrum corresponds to the median of all the normalized individual spectra including BALs. This simple approach produced a spectrum that reflects the behavior of the xA objects (see Fig. 1).

In xA sources, the $1900 \AA$ blend of the composite spectrum shows a high contribution of AlIII $\lambda 1860$ and SiIII] $\lambda 1892$ compared to CIII] $\lambda 1909$. Most of the emission on the red side of SiIII] $\lambda 1892$ can be attributed to FeIII excess in addition to the template, which is required to minimize the fit $\chi^{2}$. A fit with CIII] $\lambda 1909$ only (with no FeIII $\lambda 1914$ ) would leave a large residual 


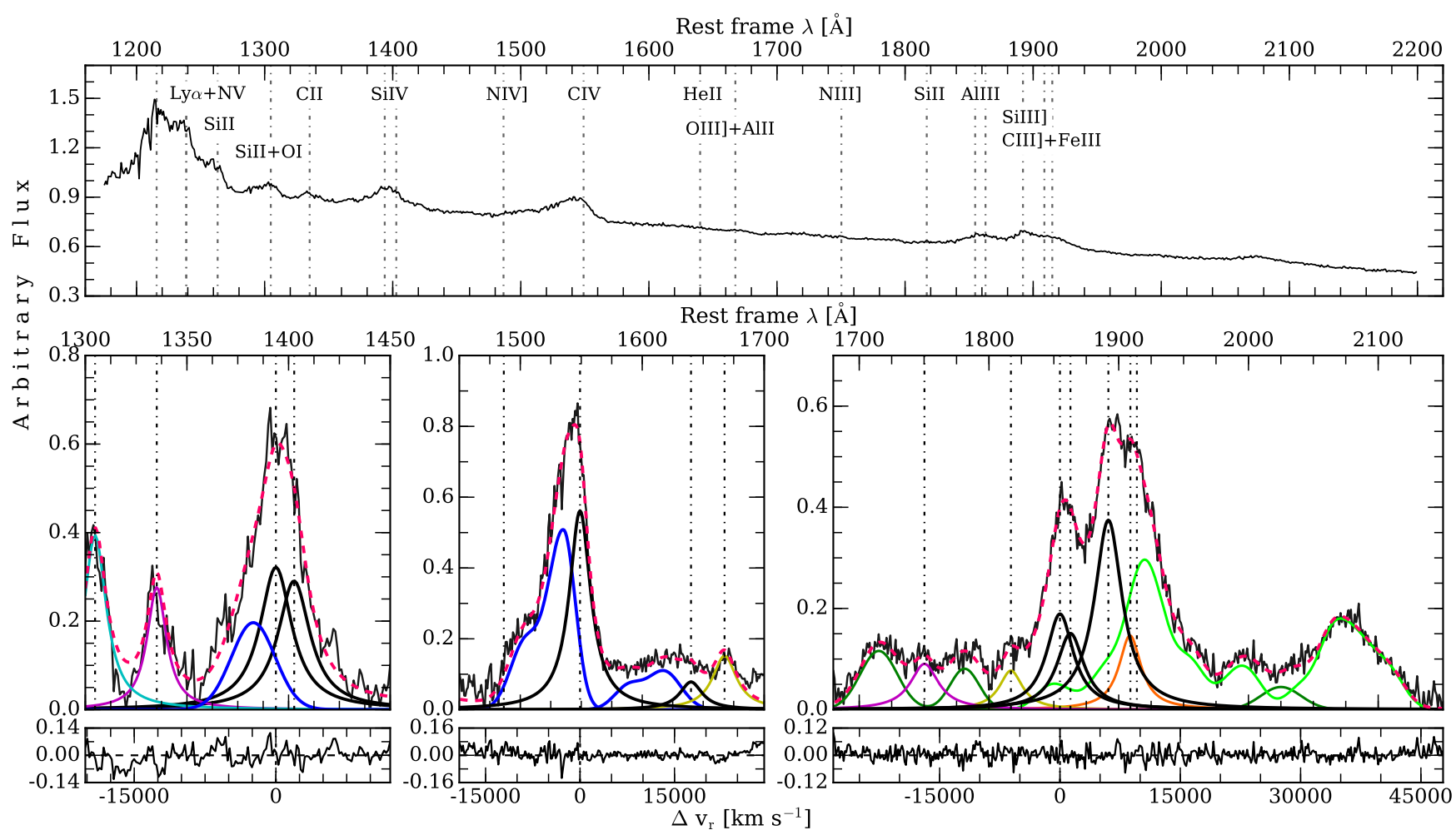

Fig. 1. Top panel: rest-frame composite spectrum (see Sect. 5.1). Abscissa corresponds to vacuum rest-frame wavelength in $\AA$, while ordinate is in arbitrary intensity. Dot-dashed vertical lines identify the position at rest-frame of the strongest emission lines. Bottom panels: multicomponent fits after continuum subtraction for the $1400 \AA$ blend, CIV $\lambda 1549$ and $1900 \AA$ blend spectral regions (Sect. 4.2). In all the panels continuous black line marks the broad component at rest-frame associated to SiIV $\lambda 1397$, CIV $\lambda 1549$, AlIII $\lambda 1860$ and SiIII] $\lambda 1892$ respectively, while the blue one corresponds to the blueshifted component associated to each emission. Dashed pink line marks the fit to the whole spectrum. Dot-dashed vertical lines correspond to the rest-frame wavelength of each emission line. In the SiIV $\lambda 1397$ spectral range, the cyan line marks the contribution of OI $\lambda 1304+$ SiII $\lambda 1306$ blend, while the magenta line corresponds to the CII $\lambda 1335$ emission line. In the CIV $\lambda 1549$ region, the yellow one corresponds to the OIII] $\lambda 1663+$ AlII $\lambda 1670$ blend. In the $1900 \AA$ Alend range, FeIII and FeII contributions are traced by dark and pale green lines respectively, magenta line marks the NIII] $\lambda 1750$ and the yellow one corresponds to the SiII $\lambda 1816$. Lower panels correspond to the residuals, abscissa is in radial velocity units $\mathrm{km} \mathrm{s}^{-1}$.

at 1915-1920 ̊. Strong FeIII emission is confirmed also by the prominent bump at $2080 \AA$ predominantly ascribed to the FeIII multiplet \#48.

On the blue side of CIV $\lambda 1549$, a smooth and shallow through is due to the combined effect of broad absorption lines that are frequent shortwards of CIV $\lambda 1549$. The emission profile of CIV $\lambda 1549$ is in any case almost fully blueshifted, as observed in the majority of sources. Also the SiIV $\lambda 1397$ median profile shows a blueshift asymmetry even if it is affected by the heavy absorptions frequently observed on the blue side of this line. The net effect is that SiIV $\lambda 1397+$ OIV appear more symmetric because their blue wings are truncated by narrow and broad absorptions. The composite also clearly shows the prominent low-ionization features associated with CII $\lambda 1335$, and OI $\lambda 1304$ blended with SiII $\lambda 1306$. CIV $\lambda 1549+$ HeII $\lambda 1640$ shows low equivalent width, close to the boundary of WLQs (see Sect. 6.6). The composite spectrum is especially helpful for the increase in $\mathrm{S} / \mathrm{N}$ that allows us to trace the broad and faint HeII $\lambda 1640$ profile, which shows a flat topped appearance. It is interpreted in the multicomponent fits as due to a strong blueshifted component, blended with a faint $\mathrm{BC}$ and with OIII] $\lambda 1663$ and AlII $\lambda 1670$.

\subsection{Consistency of selection criteria for $x A$ sources}

Using lines in the $1900 \AA$ blend, MS14 proposed that $\mathrm{xA}$ sources with $R_{\text {FeII }}>1$ show flux ratios AlIII $\lambda 1860 /$ SiIII] $\lambda 1892 \gtrsim 0.5$ and
CIII] $\lambda 1909 /$ SiIII] $\lambda 1892 \lesssim 1$. Our sample was selected considering these criteria applied to SDSS noisy spectra previously excluded by MS14. The high $\mathrm{S} / \mathrm{N}$ spectra of the GTC sample presented in this work confirm the defining criteria for the identification of the highly accreting sources in the UV range. Figure 2 shows the location of the 19 sources of our sample in the plane defined by CIII] $\lambda 1909 /$ SiIII] $\lambda 1892$ vs. AlIII $\lambda 1860 /$ SiIII] $\lambda 1892$. In order to compared the behavior of the $\mathrm{XA}$ with the rest of the Pop. A, in Fig. 2 are also represented control samples (FOS-XA, FOS-A and S14-A) described in Sect. 2.2.

Comparing the flux ratios shown by the low and high- $z$ Pop. A and xA samples, like MS14 have done, we observe a clear difference between the two kind of populations. The FOS$\mathrm{xA}$ and GTC-xA samples show flux ratios different to the ones of FOS-A and S14-A samples. The GTC-xA sources occupy a very well defined region in the bottom right side of the panel, while the Pop. A sample occupy the left top space. Two of the objects (SDSSJ222753.07-092951.7 and SDSSJ125659.79$033813.8, \sim 10 \%$ of our sample) do not rigorously satisfy the selection criteria, although they present similar spectral properties to ones observed in the rest of the sample. The measured line ratios are actually borderline: the AlIII $\lambda 1860 / \mathrm{SiIII}] \lambda 1892$ ratio of the BAL SDSSJ125659.79-033813.8 is $\approx 0.40 \pm 0.10$, the CIII] $\lambda 1909 /$ SiIII] $\lambda 1892$ ratio of SDSSJ222753.07-092951.7 is $\approx 1.36 \pm 0.23$. Therefore the two quasars will be considered along with all other GTC-xA sources. 


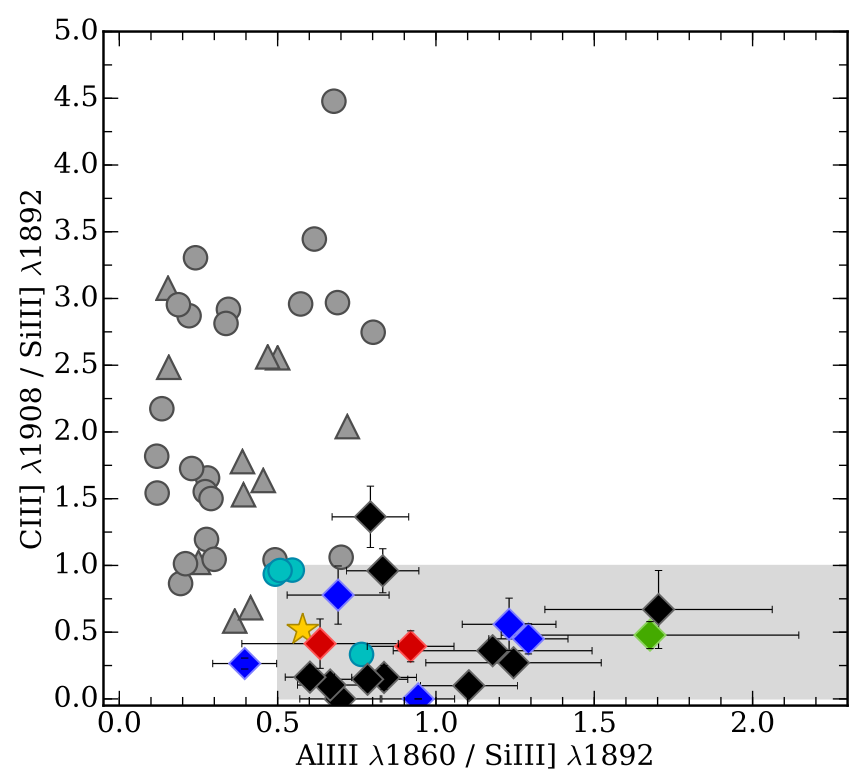

Fig. 2. Relation between intensity ratios AlIII $\lambda 1860 /$ SiIII] $\lambda 1892$ and CIII] $\lambda 1909 /$ SiIII] $\lambda 1892$. Black, blue, green and red diamonds correspond to normal, BAL, mini-BAL and Radio-Loud quasars respectively for the present GTC-xA sample. Gray and cyan dots correspond to the FOS-A and FOS-xA sample respectively. Gray triangles correspond to S14-A sample. Yellow star marks the position of I Zw 1. Gray area represents the parameter space occupied by xA sources.

\section{3. $1900 \AA$ blend}

Table 4 reports emission lines measurements corresponding to the $1900 \AA$ blend region: AlIII $\lambda 1860$, SiIII] $\lambda 1892$, CIII] $\lambda 1909$, NIII] $\lambda 1750$ and SiII $\lambda 1816$. The first column lists the SDSS name. The equivalent width $(W)$, flux $(F)$ and FWHM of AlIII $\lambda 1860$ are reported in Cols. 2, 3, and 4, respectively. Equivalent width and flux are reported for SiIII] 11892 in Cols. 5 and 6, since its FWHM is assumed equal to the one of AlIII $\lambda 1860 . W, F$ and FWHM of CIII] $\lambda 1909$ are reported in Cols. 7, 8 and 9, respectively. They are followed by the flux of the two faint features outside of the blend: NIII] $\lambda 1750$ and SiII $\lambda 1816$ (Cols. 10 and 11). All the parameters have uncertainties at $1 \sigma$ confidence level, which were obtained from the MC simulations. The MC method followed to estimate the uncertainties is explained in Appendix B.

In several instances the AlIII $\lambda 1860$ doublet is partly resolved and is assumed to be at the rest-frame along with SiIII] $\lambda 1892$. In general, the AlIII $\lambda 1860$ flux, FWHM and $W$ can be estimated with good accuracy as the doublet can be efficiently deblended save for the cases that the lines are very broad $\left(F W H M \gtrsim 6000 \mathrm{~km} \mathrm{~s}^{-1}\right)$. AlIII $\lambda 1860$ is well fit with a symmetric and unshifted Lorentzian. Typical uncertainties in $F W H M$ are $\lesssim 10 \%$. Only in one case (SDSSJ084036.16+235524.7) there is evidence for a blue asymmetry in the $1900 \AA$ Alend although the AlIII $\lambda 1860$ BC can be isolated with good accuracy. The fraction of the blueshifted to the total AlIII emission would of $\approx 0.25$, small compared to a median value of $\approx 0.46$ for CIV. The presence of a blue shifted component in AlIII $\lambda 1860$ is a rare occurrence. Only in one source of the S17 sample shows evidence of a blueward AlIII $\lambda 1860$ excess (HE0359-3959, Martínez-Aldama et al. 2017).

The peak of the $1900 \AA$ blend is often not in correspondence of the CIII] $\lambda 1909$ wavelength. If the blend of SiIII], CIII] and FeIII is peaked, the peak is preferentially redshifted beyond $1909 \AA$. The $1900 \AA$ blend profile may show excess emission on its red side (see e.g., SDSSJ021606.41+011509.5, Fig. A.3). In these cases, we suggest that the main contribution is coming from the FeIII $\lambda 1914$ line, modeled with a Lorentzian in excess to the FeIII template (Sects. 5.6 and 6.4), or by unresolved FeIII emission.

The weaker lines NIII] $\lambda 1750$ and SiII $\lambda 1816$ are detected in about two thirds of the sample, for example they are both prominent in the case of the quasar SDSSJ021606.41+011509.5. The NIII] $\lambda 1750$ line shows a wide range in $W$. It is not detected in four sources but the detections show $W$ in the range $0.5-5 \AA$.

\subsection{CIV $\lambda 1549$ and Hell $\lambda 1640$}

Measurements associated with the emission lines of the CIV $\lambda 1549$ spectral region are reported in Table 5. The first column lists the SDSS name. Column 2 reports the rest-frame specific continuum flux $\left(f_{\lambda}\right)$ at $1350 \AA$. No error estimate is provided; the inter calibration with different standard star spectra suggests an uncertainty around $10 \%$, which should include all main source of errors in the absolute flux scale. Columns 3-7 tabulate $W$, flux, FWHM, centroid at half intensity $\left(c\left(\frac{1}{2}\right)\right)$ and asymmetry index (A.I.) of the CIV $\lambda 1549$ total profile (broad plus blueshifted components). Columns 8 and 9 list the equivalent width and $F$ of the broad component of CIV. Its FWHM is not reported, because it is assumed to be the same as FWHM AlIII $\lambda 1860$, due to the BC have to be emitted by the same zone. The equivalent width, $F$, FWHM and $c\left(\frac{1}{2}\right)$ of the blueshifted component of CIV are given in Cols. 10-13, respectively.

Table 6 reports the measurements associated with HeII $\lambda 1640$ and contaminant lines. Columns 2-7 list the $W$ and flux of HeII $\lambda 1640$ total profile, HeII $\lambda 1640 \mathrm{BC}$ and HeII $\lambda 1640$ blue shifted component respectively. Column 8 lists the OIII] $\lambda 1663+$ AlII $\lambda 1670$ flux. The last column reports the NIV] $\lambda 1486$ flux. The NIV] $\lambda 1486$ flux is highly uncertain due to absorption lines and to the blending with the blue wing of CIV, then in many of the cases only upper limits are reported.

On average, the HeII $\lambda 1640$ total flux is $\sim 20 \%$ that of CIV $\lambda 1549$. Since the typical $W \operatorname{CIV} \lambda 1549 \approx 10-15 \AA$, the typical $W$ HeII $\lambda 1640$ is around $\approx 3 \AA$ distributed over a broad profile with $4000 \lesssim F W H M \lesssim 11000 \mathrm{~km} \mathrm{~s}^{-1}$. The weakness of the HeII $\lambda 1640$ emission with respect to CIV $\lambda 1549$, with CIV $\lambda 1549 /$ HeII $\lambda 1640 \approx 5-6 \gtrsim 3$ is out of the question. It is important however to stress that our approach tends to maximize the contribution of the $\operatorname{CIV}_{\mathrm{BC}}$ to the total CIV $\lambda 1549$ emission. This may lead to an overestimation of the CIV $\lambda 1549 / \mathrm{HeII} \lambda 1640$ BC ratio. Nevertheless, in several cases (e.g., SDSS J214009.1064403.9) we observed a significant CIV $\lambda 1549$ emission at restframe, implying that the CIV $\lambda 1549 / \mathrm{HeII} \lambda 1640$ estimate $\approx 5-6$ is a safe one. We mention the CIV $\lambda 1549 / \mathrm{HeII} \lambda 1640$ ratio because its value is rather high for the low ionization parameter expected in the emitting regions, if metallicity is solar or slightly supersolar (Sect. 6.3, N12).

An important feature in our spectra involves the strong blueshifts and asymmetries associated with the HILs. In $\sim 50 \%$ of our sample, the CIV blueshifted component contributes $\sim 50 \%$ of the total profile. HeII $\lambda 1640$ also shows a highly blueshifted component, contributing $\sim 60 \%$ of the total flux. The A.I. values also support the presence of outflows: the blueshifted component pumped by radiative forces is more prominent than the virial component located at rest-frame. This is a major difference between high-ionization lines (e.g., CIV 21549 ) on the one side, and low and intermediate-ionization lines on the other (e.g., AlIII 1860). The last one are basically symmetric and show no evidence of large shifts with respect to the rest-frame.

An indication of the strength of the outflow is provided by the shift amplitude of the blue shifted component or by the shift amplitude of the total CIV profile measured by the 
Table 4. Measurements in the $1900 \AA ̊$ blend region.

\begin{tabular}{|c|c|c|c|c|c|c|c|c|c|c|}
\hline \multirow{2}{*}{$\begin{array}{l}\text { SDSS identification } \\
\text { (1) }\end{array}$} & \multicolumn{3}{|c|}{ AlIII 1860} & \multicolumn{2}{|c|}{ SiIII] 1892} & \multicolumn{3}{|c|}{ CIII] 1909} & \multirow{2}{*}{$\begin{array}{c}\text { NIII] } 1750 \\
F \\
(10)\end{array}$} & \multirow{2}{*}{$\begin{array}{c}\text { SiII } 1816 \\
F \\
(11)\end{array}$} \\
\hline & $\begin{array}{l}W \\
(2) \\
\end{array}$ & $\begin{array}{c}F \\
(3) \\
\end{array}$ & $\begin{array}{c}F W H M \\
\text { (4) }\end{array}$ & $\begin{array}{c}W \\
(5) \\
\end{array}$ & $\begin{array}{c}F \\
(6) \\
\end{array}$ & $\begin{array}{c}W \\
(7) \\
\end{array}$ & $\begin{array}{c}F \\
(8) \\
\end{array}$ & $\begin{array}{c}F W H M \\
(9)\end{array}$ & & \\
\hline SDSSJ000807.27-103942.7 & $9.6 \pm 1.2$ & $11 \pm 0.8$ & $5410 \pm 630$ & $12.1 \pm 1.9$ & $13.2 \pm 1.6$ & $11.9 \pm 1.9$ & $12.7 \pm 1.6$ & $4810 \pm 790$ & $6.5 \pm 0.9$ & $3.1 \pm 1.0$ \\
\hline SDSSJ004241.95+002213.9 & $9.6 \pm 1.3$ & $25.2 \pm 2.2$ & $5060 \pm 340$ & $11.8 \pm 1.5$ & $30.2 \pm 2.5$ & $1.9 \pm 1.0$ & $4.9 \pm 2.6$ & $3060 \pm 1620$ & $4.8 \pm 1.5$ & $2.2 \mathrm{u}$ \\
\hline SDSSJ021606.41+011509.5 & $11.3 \pm 1.3$ & $33.2 \pm 1.7$ & $3190 \pm 220$ & $9.0 \pm 1.2$ & $25.7 \pm 2.1$ & $3.7 \pm 1.0$ & $11.6 \pm 2.8$ & $2690 \pm 850$ & $7.4 \pm 1.8$ & $8.1 \pm 1.5^{a}$ \\
\hline SDSSJ024154.42-004757.5 & $5.1 \pm 1.1$ & $9.5 \pm 1.9$ & $3340 \pm 320$ & $4.2 \pm 0.6$ & $7.7 \pm 0.8$ & $1.2 \mathrm{u}$ & $2.1 \mathrm{u}$ & & $\ldots$ & $6.6 \pm 0.8$ \\
\hline SDSSJ084036.16+235524.7 & $6.1 \pm 1.2$ & $11.5 \pm 2$ & $3420 \pm 380$ & $5.3 \pm 1.2$ & $9.8 \pm 2$ & $2.0 \pm 0.9$ & $3.5 \pm 1.6$ & $2850 \pm 420$ & & $3.3 \pm 0.6^{a}$ \\
\hline SDSSJ101822.96+203558.6 & $8.5 \pm 1.2$ & $26.7 \pm 2.8$ & $4870 \pm 340$ & $11.1 \pm 1.8$ & $33.4 \pm 4.2$ & $1.7 \mathrm{u}$ & $4.9 \mathrm{u}$ & - $00-2.0$ & $3 \mathrm{u}$ & $6 \pm 2.4$ \\
\hline SDSSJ103527.40+445435.6 & $7.9 \pm 1.4$ & $19 \pm 2.8$ & $7060 \pm 480$ & $11.5 \pm 1.7$ & $27.3 \pm 2.9$ & & $\ldots$ & $\ldots$ & $\ldots$ & \\
\hline SDSSJ105806.16+600826.9 & $5.1 \pm 0.8$ & $12.8 \pm 1.6$ & $4180 \pm 650$ & $7.9 \pm 1.1$ & $19.2 \pm 1.7$ & $0.8 \mathrm{u}$ & $2 \mathrm{u}$ & & $4.2 \pm 2.1$ & $6.7 \pm 1.3$ \\
\hline SDSSJ110022.53+484012.6 & $9.1 \pm 0.8$ & $21.8 \pm 2.2$ & $4900 \pm 390$ & $15.6 \pm 2.0$ & $36.3 \pm 2.9$ & $2.6 \pm 1.2$ & $5.9 \pm 2.7$ & $3510 \pm 1970$ & $5.3 \pm 1.3$ & $2.4 \pm 1.7$ \\
\hline SDSSJ125659.79-033813.8 & $13.4 \pm 3.0^{a}$ & $24.1 \pm 4.9^{a}$ & $5950 \pm 600$ & $34.9 \pm 6.4$ & $60.9 \pm 9.4$ & $9.5 \pm 4.3$ & $16.2 \pm 7.2$ & $4000 \pm 2090$ & $7.1 \pm 4.5^{a}$ & $7.8 \pm 3.7^{a}$ \\
\hline SDSSJ131132.92+052751.2 & $5.8 \pm 0.7$ & $16.4 \pm 1.3$ & $3760 \pm 250$ & $6.2 \pm 0.8$ & $17.3 \pm 1.6$ & & & $\ldots$ & & \\
\hline SDSSJ143525.31+400112.2 & $4 \pm 0.5$ & $25.8 \pm 2.3$ & $3350 \pm 270$ & $3.8 \pm 0.6$ & $23.4 \pm 2.5$ & $0.4 \mathrm{u}$ & $2.3 \mathrm{u}$ & & $5.3 \pm 2.8$ & $4.4 \pm 1.9$ \\
\hline SDSSJ144412.37+582636.9 & $13 \pm 2.7$ & $12.5 \pm 2.3$ & $8080 \pm 1560$ & $7.9 \pm 1.9$ & $7.5 \pm 1.6$ & $3.8 \mathrm{u}$ & $3.6 \mathrm{u}$ & & $5.8 \pm 0.5$ & $1.6 \pm 1.0$ \\
\hline SDSSJ151258.36+352533.2 & $4.1 \pm 0.6$ & $10.8 \pm 1.1$ & $2780 \pm 330$ & $4.6 \pm 0.7$ & $11.8 \pm 1.2$ & $1.9 \pm 0.6$ & $4.6 \pm 1.3$ & $2110 \pm 700$ & $5.8 \pm 1.2$ & $5.3 \pm 1.2$ \\
\hline SDSSJ214009.01-064403.9 & $7.7 \pm 0.9$ & $13.9 \pm 0.9$ & $2620 \pm 160$ & $6.3 \pm 0.9$ & $11.3 \pm 1.1$ & $3.5 \pm 1.2$ & $7.4 \pm 2.1$ & $3500 \pm 1150$ & $0.8 \mathrm{u}$ & $1.5 \pm 0.7$ \\
\hline SDSSJ220119.62-083911.6 & $6.3 \pm 1.1$ & $26.7 \pm 4$ & $7250 \pm 1050$ & $9.3 \pm 1.9$ & $38.6 \pm 6.9$ & $7.4 \pm 1.8$ & $30 \pm 6.5$ & $6110 \pm 1670$ & $6.7 \pm 2.4$ & \\
\hline SDSSJ222753.07-092951.7 & $6 \pm 0.8$ & $11.2 \pm 1$ & $4500 \pm 420$ & $7.8 \pm 1.2$ & $14.1 \pm 1.7$ & $10.8 \pm 1.6$ & $19.2 \pm 2.2$ & $4550 \pm 590$ & $2.9 \pm 1.2$ & $0.5 \mathrm{u}$ \\
\hline SDSSJ233132.83+010620.9 & $5.5 \pm 1.5^{a}$ & $38.5 \pm 10.2^{a}$ & $6310 \pm 1640^{a}$ & $8.8 \pm 2.7$ & $60.7 \pm 17.4$ & $3.7 \pm 1.3$ & $25.2 \pm 8.6$ & $5530 \pm 4700$ & $10.2 \pm 5.5^{a}$ & $6.6 \mathrm{u}^{a}$ \\
\hline SDSSJ234657.25+145736.0 & $6.9 \pm 0.8$ & $21.1 \pm 1.3$ & $3660 \pm 560$ & $4.1 \pm 0.9$ & $12.4 \pm 2.5$ & $2.8 \pm 1.1$ & $8.3 \pm 3.2$ & $3040 \pm 1450$ & $2.8 \pm 1.4^{a}$ & $5.3 \pm 1.4^{a}$ \\
\hline
\end{tabular}

Notes. Columns are as follows: (1) SDSS name. (2), (5) and (7) report the equivalent width in units of $\AA$. (3), (6), (8), (10) and (11) list fluxes in units of $\mathrm{erg} \mathrm{s}^{-1} \mathrm{~cm}^{-2}$. (4) and (9) correspond to the FWHM of AlIII and CIII]in units of $\mathrm{km} \mathrm{s}^{-1}$. ${ }^{(a)}$ Means that measurement is contaminated by absorption lines. A $u$ letter marks an upper limit to the measurement.

Table 5. Measurements on CIVג1549 region.

\begin{tabular}{|c|c|c|c|c|c|c|c|c|c|c|c|c|}
\hline \multirow{2}{*}{$\begin{array}{c}\text { SDSS identification } \\
\text { (1) }\end{array}$} & \multirow{2}{*}{$\begin{array}{c}f_{\lambda}(1350 \AA) \\
(2) \\
\end{array}$} & \multicolumn{5}{|c|}{ CIV $1549_{\text {TOTAL }}$} & \multicolumn{2}{|c|}{ CIV $1549_{\mathrm{BC}}$} & \multicolumn{4}{|c|}{ CIV $1549_{\text {BLUE }}$} \\
\hline & & $\begin{array}{l}W \\
(3) \\
\end{array}$ & $\begin{array}{c}F \\
(4) \\
\end{array}$ & $\begin{array}{c}F W H M \\
(5)\end{array}$ & $\begin{array}{l}c\left(\frac{1}{2}\right) \\
(6)\end{array}$ & $\begin{array}{l}\text { A.I. } \\
(7)\end{array}$ & $\begin{array}{l}W \\
(8) \\
\end{array}$ & $\begin{array}{c}F \\
(9) \\
\end{array}$ & $\begin{array}{c}W \\
(10)\end{array}$ & $\begin{array}{c}F \\
(11) \\
\end{array}$ & $\begin{array}{c}F W H M \\
(12)\end{array}$ & $\begin{array}{l}c\left(\frac{1}{2}\right) \\
(13)\end{array}$ \\
\hline SDSSJ000807.27-103942.7 & 2.9 & $37.7 \pm 6.6^{a}$ & $71.9 \pm 10.4^{a}$ & $6200 \pm 420$ & $-1130 \pm 210$ & $-0.044 \pm 0.103$ & $27.4 \pm 6.0^{a}$ & $51.8 \pm 10.1^{a}$ & $10.3 \pm 5.3^{a}$ & $20.1 \pm 10.2^{a}$ & $4770 \pm 300$ & $-2740 \pm 150$ \\
\hline SDSSJ004241.95+002213.9 & 3.7 & $19.5 \pm 4.3$ & $61.8 \pm 12.2$ & $10080 \pm 340$ & $-4530 \pm 170$ & $-0.438 \pm 0.087$ & $5.2 \pm 4.4$ & $16.4 \pm 13.6$ & $14.3 \pm 2.7$ & $45.4 \pm 7.4$ & $9550 \pm 250$ & $-5170 \pm 130$ \\
\hline SDSSJ021606.41+011509.5 & 3.5 & $19.1 \pm 2.6^{b}$ & $62.4 \pm 5.5^{b}$ & $4130 \pm 230$ & $-850 \pm 110$ & $-0.074 \pm 0.086$ & $13.4 \pm 2.5$ & $43.8 \pm 6.8$ & $5.7 \pm 1.1^{b}$ & $18.6 \pm 3^{b}$ & $3020 \pm 160$ & $-1760 \pm 80$ \\
\hline SDSSJ024154.42-004757.5 & 3.1 & $10.0 \pm 1.8^{a}$ & $25.4 \pm 3.7^{a}$ & $6830 \pm 450$ & $-2420 \pm 220$ & $-0.375 \pm 0.073$ & $3.9 \pm 1.3^{a}$ & $9.8 \pm 3.2^{a}$ & $6.1 \pm 1.9^{a}$ & $15.6 \pm 4.7^{a}$ & $6570 \pm 380$ & $-3560 \pm 1909$ \\
\hline SDSSJ084036.16+235524.7 & 3.1 & $14.4 \pm 2.2$ & $38.1 \pm 4.5$ & $6630 \pm 370$ & $-1670 \pm 180$ & $-0.378 \pm 0.070$ & $9.9 \pm 2.4$ & $26.6 \pm 5.9$ & $4.5 \pm 2$ & $11.5 \pm 4.8$ & $4590 \pm 240$ & $-3850 \pm 120$ \\
\hline SDSSJ101822.96+203558.6 & 4.4 & $12.1 \pm 2.7^{a}$ & $47.5 \pm 9.5^{a}$ & $9580 \pm 390$ & $-3990 \pm 200$ & $-0.533 \pm 0.069$ & $2.6 \pm 1.7^{a}$ & $10.0 \pm 6.6^{a}$ & $9.5 \pm 2.1^{a}$ & $37.5 \pm 7.4^{a}$ & $9770 \pm 320$ & $-4520 \pm 160$ \\
\hline SDSSJ103527.40+445435.6 & 3.0 & $12.2 \pm 4.2^{a}$ & $33.4 \pm 11.1^{a}$ & $9390 \pm 450$ & $-3460 \pm 230$ & $0.193 \pm 0.073$ & $5.9 \pm 3.3$ & $16.2 \pm 8.8$ & $6.3 \pm 2.5^{a}$ & $17.2 \pm 6.7^{a}$ & $6350 \pm 320$ & $-5050 \pm 160$ \\
\hline SDSSJ105806.16+600826.9 & 4.6 & $11.4 \pm 2.2$ & $39.3 \pm 6.4$ & $6880 \pm 400$ & $-2890 \pm 200$ & $-0.405 \pm 0.076$ & $1.5 \pm 1.4$ & $5.0 \pm 4.8$ & $9.9 \pm 1.9$ & $34.3 \pm 5.4$ & $6840 \pm 380$ & $-3160 \pm 190$ \\
\hline SDSSJ110022.53+484012.6 & 3.7 & $24.3 \pm 2.8$ & $75.9 \pm 4.1$ & $8010 \pm 540$ & $-4430 \pm 270$ & $-0.203 \pm 0.071$ & $7.1 \pm 1.7$ & $22.0 \pm 4.9$ & $17.2 \pm 2$ & $53.9 \pm 3.4$ & $7140 \pm 410$ & $-5120 \pm 210$ \\
\hline SDSSJ125659.79-033813.8 & 2.8 & $47.4 \pm 9.9^{a}$ & $111.2 \pm 20.4^{a}$ & $5650 \pm 460$ & $-2020 \pm 230$ & $-0.186 \pm 0.091$ & $25.8 \pm 11.8$ & $60.5 \pm 27.1^{a}$ & $21.6 \pm 8.2^{a}$ & $50.7 \pm 18.5$ & $5000 \pm 400$ & $-2950 \pm 200$ \\
\hline SDSSJ131132.92+052751.2 & 2.0 & $6.6 \pm 0.9^{\mathrm{a}, \mathrm{b}}$ & $17.6 \pm 1.8^{\mathrm{a}, \mathrm{b}}$ & $5700 \pm 320$ & $-1180 \pm 160$ & $-0.220 \pm 0.088$ & $4.7 \pm 0.9^{a}$ & $12.5 \pm 2.2^{a}$ & $1.9 \pm 0.7^{b}$ & $5.1 \pm 1.7^{b}$ & $4320 \pm 220$ & $-2710 \pm 110$ \\
\hline SDSSJ143525.31+400112.2 & 10.3 & $9.9 \pm 1.3^{a}$ & $85.2 \pm 7.2^{a}$ & $8210 \pm 680$ & $-3090 \pm 340$ & $-0.214 \pm 0.074$ & $3.7 \pm 1.2$ & $31.6 \pm 9.5$ & $6.2 \pm 1.0^{a}$ & $53.6 \pm 6.8^{a}$ & $6990 \pm 430$ & $-4330 \pm 220$ \\
\hline SDSSJ144412.37+582636.9 & 1.0 & $13.9 \pm 1.8^{a}$ & $15.0 \pm 1.2^{a}$ & $8570 \pm 450$ & $-1990 \pm 230$ & $0.020 \pm 0.085$ & $9.0 \pm 2.0^{a}$ & $9.7 \pm 2^{a}$ & $4.9 \pm 1.7^{a}$ & $5.3 \pm 1.7^{a}$ & $6310 \pm 320$ & $-3480 \pm 160$ \\
\hline SDSSJ151258.36+352533.2 & 4.7 & $8.8 \pm 1.4^{a}$ & $32.6 \pm 4.0^{a}$ & $5300 \pm 220$ & $-1880 \pm 110$ & $-0.022 \pm 0.090$ & $3.3 \pm 1.1^{a}$ & $12.1 \pm 3.7^{a}$ & $5.5 \pm 1.2^{a}$ & $20.5 \pm 4.2^{a}$ & $3990 \pm 200$ & $-2630 \pm 100$ \\
\hline SDSSJ214009.01-064403.9 & 1.9 & $16.1 \pm 2.4^{b}$ & $29.7 \pm 3.4^{b}$ & $5260 \pm 770$ & $-1170 \pm 380$ & $-0.424 \pm 0.069$ & $10.8 \pm 2.6^{b}$ & $20.0 \pm 4.3^{b}$ & $5.3 \pm 1.8^{b}$ & $9.7 \pm 3.2^{b}$ & $5350 \pm 270$ & $-3770 \pm 140$ \\
\hline SDSSJ220119.62-083911.6 & 5.5 & $18.4 \pm 3.8^{b}$ & $103.4 \pm 19.0^{b}$ & $4430 \pm 510$ & $-830 \pm 250$ & $0.002 \pm 0.092$ & $15.2 \pm 4.9^{b}$ & $85.7 \pm 26.4^{b}$ & $3.2 \pm 2.7^{b}$ & $17.7 \pm 15^{b}$ & $2920 \pm 170$ & $-1160 \pm 90$ \\
\hline SDSSJ222753.07-092951.7 & 2.8 & $31.9 \pm 5.5$ & $77.9 \pm 11$ & $5260 \pm 410$ & $-1190 \pm 200$ & $-0.277 \pm 0.051$ & $16.3 \pm 4.8$ & $39.5 \pm 11.2$ & $15.7 \pm 6.8$ & $38.4 \pm 16.2$ & $5820 \pm 420$ & $-2340 \pm 210$ \\
\hline SDSSJ233132.83+010620.9 & 8.3 & $21.4 \pm 4.4$ & $174.2 \pm 35.8$ & $11230 \pm 700$ & $-2430 \pm 350$ & $-0.333 \pm 0.097$ & $16.1 \pm 5$ & $132.0 \pm 39.1$ & $5.3 \pm 1.6$ & $42.2 \pm 12.4$ & $6990 \pm 380$ & $-6960 \pm 190$ \\
\hline SDSSJ234657.25+145736.0 & 4.4 & $11.1 \pm 1.7^{a}$ & $41.5 \pm 4.6^{a}$ & $6390 \pm 800$ & $-1420 \pm 400$ & $-0.392 \pm 0.071$ & $7.2 \pm 1.5^{a}$ & $26.9 \pm 4.9^{a}$ & $3.9 \pm 1.5^{a}$ & $14.6 \pm 5.3^{a}$ & $6670 \pm 340$ & $-4750 \pm 170$ \\
\hline
\end{tabular}

Notes. Columns are as follows: (1) SDSS name. (2) Continuum flux measured at $1350 \AA$. (3), (8) and (10) report the equivalent width in unit of $\AA$. (4), (9) and (11) list line fluxes in units of $\mathrm{erg} \mathrm{s}^{-1} \mathrm{~cm}^{-2}$. (5) and (12) list the FWHM of the corresponding line in $\mathrm{km} \mathrm{s} \mathrm{s}^{-1}$. (6) and (13) report the centroid at half intensity in units of $\mathrm{km} \mathrm{s}^{-1}$. (7) lists the asymmetry index (A.I.). ${ }^{(a)}$ Measurement contaminated by narrow absorption lines. ${ }^{(b)}$ Measurement contaminated by broad absorption lines.

centroid at half intensity, $c\left(\frac{1}{2}\right)$. The most negative values indicate stronger blueshifts suggesting stronger outflows. BAL quasars tend to show smaller values of $c\left(\frac{1}{2}\right)$ (closer to 0 ) in the CIV profile, due to the presence of the absorption features in the blue side of the profile. The non-BAL quasars show values $1000 \lesssim\left|c\left(\frac{1}{2}\right)\right| \lesssim 5000 \mathrm{~km} \mathrm{~s}^{-1}$. HeII $\lambda 1640$ shows even stronger asymmetries than CIV $\lambda 1549$, although they could be related to the faintness of HeII $\lambda 1640$ and the deblending uncertain of broad and blue components. The strong outflows could be important for feedback effects on the host galaxy (Sect. 6.7).

\subsection{SiIv $1397+O \operatorname{Iv}] \lambda 1402$}

Table 7 reports the values of the emission lines in the Sirv $\lambda 1397+$ OIV spectral region (1400 $\AA$ blend). The blue side of the $1400 \AA$ spectral region is seriously affected by absorption lines. The SiIV $\lambda 1397+$ OIV blend is formed by highionization lines. A blueshift asymmetry is expected in the profile, but in $\sim 40 \%$ of the sample the blueshifted component can not be detected due to strong absorption lines. In the rest of the sample, the measurements of the $c\left(\frac{1}{2}\right)$ and FWHM are also affected to the ever-present absorptions. CII $\lambda 1335$ and $\mathrm{OI}+\mathrm{SII} \lambda 1304$ are also affected by the absorption. In the majority of the sample, they cannot be detected or their flux uncertainty is large.

\subsection{The Fell and Fell emission}

FeII emission is usually not very strong in the range 1700$2100 \AA$, even in the case of the strongest optical FeII emitters. Only a few features are detected at $\sim 1715 \AA, 1785 \AA$ and $2020 \AA$. 
Table 6. Measurements on HeII $\lambda 1640$ region.

\begin{tabular}{|c|c|c|c|c|c|c|c|c|}
\hline \multirow{2}{*}{$\begin{array}{l}\text { SDSS identification } \\
\text { (1) }\end{array}$} & \multicolumn{2}{|c|}{ HeII 1640 TOTAL } & \multicolumn{2}{|c|}{ HeII $1640_{\mathrm{BC}}$} & \multicolumn{2}{|c|}{ HeII $1640_{\text {BLUE }}$} & \multirow{2}{*}{$\begin{array}{c}\text { AlII + OIII] } 1667 \\
F \\
(8) \\
\end{array}$} & \multirow{2}{*}{$\begin{array}{c}\text { NIV] } 1486 \\
F \\
(9)\end{array}$} \\
\hline & $\begin{array}{l}W \\
(2)\end{array}$ & $\begin{array}{c}F \\
(3)\end{array}$ & $\begin{array}{l}W \\
(4)\end{array}$ & $\begin{array}{c}F \\
(5)\end{array}$ & $\begin{array}{l}W \\
(6)\end{array}$ & $\begin{array}{c}F \\
(7)\end{array}$ & & \\
\hline SDSSJ000807.27-103942.7 & $7.9 \mathrm{u}$ & $13.2 \mathrm{u}$ & $2.5 \mathrm{u}$ & $4.0 \mathrm{u}$ & $5.4 \mathrm{u}$ & $9.2 \mathrm{u}$ & $8.5 \pm 4.1$ & $2.4 \mathrm{u}^{a}$ \\
\hline SDSSJ004241.95+002213.9 & $2.7 \pm 2.4$ & $8.3 \pm 7.2$ & $1.0 \mathrm{u}$ & $3.1 \mathrm{u}$ & $1.7 \pm 1.2$ & $5.2 \pm 3.6$ & $2.7 \mathrm{u}$ & $7.8 \mathrm{u}$ \\
\hline SDSSJ021606.41+011509.5 & $2.1 \pm 1.9$ & $6.6 \pm 5.8$ & 1.3: & 4.1: & 0.8: & 2.5: & $2.2 \pm 1.4$ & . \\
\hline SDSSJ024154.42-004757.5 & $3.3 \pm 0.9$ & $7.7 \pm 2$ & 0.5 & 1.1: & $2.8 \pm 0.9$ & $6.6 \pm 2.1$ & $4.5 \mathrm{u}$ & $5.5 \pm 3.5^{a}$ \\
\hline SDSSJ084036.16+235524.7 & $2.3 \pm 1.6$ & $5.6 \pm 3.7$ & $1.2 \pm 1$ & $3.0 \pm 2.5$ & $1.1 \pm 1$ & 2.6 u 2.2 & $2.5 \pm 1.9$ & $3.7 \mathrm{u}$ \\
\hline SDSSJ101822.96+203558.6 & $2.2 \mathrm{u}$ & $8.2 \mathrm{u}$ & $0.7 \mathrm{u}$ & $2.5 \mathrm{u}$ & $1.5 \pm 0.7$ & $5.7 \pm 2.5$ & $5.5 \mathrm{u}$ & $8 \mathrm{u}$ \\
\hline SDSSJ103527.40+445435.6 & $2.5 \mathrm{u}$ & $6.5 \pm 2.3$ & $1.6 \mathrm{u}$ & $4.2 \mathrm{u}$ & $0.9 \pm 0.4$ & $2.3 \pm 1$ & $5.1 \pm 2.6$ & $16.2 \mathrm{u}^{a}$ \\
\hline SDSSJ105806.16+600826.9 & $1.1 \mathrm{u}$ & $3.2 \mathrm{u}$ & 0.5 : & 1.5: & $0.6 \mathrm{u}$ & $1.7 \mathrm{u}$ & $3 \mathrm{u}$ & $10 \mathrm{u}$ \\
\hline SDSSJ110022.53+484012.6 & $2.8 \pm 1.0^{a}$ & $8.2 \pm 2.8^{a}$ & $1.0^{a}$ & $2.9^{a}$ & $1.8 \pm 0.9$ & $5.3 \pm 2.6$ & $1.7 \mathrm{u}$ & $\ldots$ \\
\hline SDSSJ125659.79-033813.8 & $7.0 \mathrm{u}^{a}$ & $15.5 \mathrm{u}^{a}$ & 2.7: & 6.0: & $4.3 \mathrm{u}^{a}$ & $9.5 \mathrm{u}^{a}$ & $5.5 \mathrm{u}$ & $\ldots$ \\
\hline SDSSJ131132.92+052751.2 & $4.0 \pm 1.0^{a}$ & $10.4 \pm 2.3^{a}$ & $1.2 \pm 0.9$ & $3.1 \pm 2.3$ & $2.8 \pm 1.1^{a}$ & $7.3 \pm 2.7^{a}$ & $6.7 \pm 2.5 \mathrm{u}^{a}$ & \\
\hline SDSSJ143525.31+400112.2 & $2.3 \pm 0.9^{a}$ & $18.2 \pm 6.5^{a}$ & $1.3 \pm 0.8^{a}$ & $10.3 \pm 5.9^{a}$ & $1 \pm 0.5$ & $7.9 \pm 3.7$ & $9.2 \pm 4.1^{a}$ & $5.8 \mathrm{u}^{a}$ \\
\hline SDSSJ144412.37+582636.9 & $1.3 \pm 0.7$ & $4.0 \pm 2.5$ & $1.1 \pm 0.8$ & $2.9 \pm 2.2$ & 0.2 : & 1.1: & $4.9 \mathrm{u}$ & $5.7 \pm 4.2 \mathrm{u}^{a}$ \\
\hline SDSSJ151258.36+352533.2 & $2.0 \mathrm{u}^{a}$ & $6.6 \mathrm{u}^{a}$ & $1 \pm 0.7^{a}$ & $3.2 \pm 2.3^{a}$ & $1 \mathrm{u}^{a}$ & $3.4 \mathrm{u}^{a}$ & $5.3 \pm 4.1$ & $\ldots$ \\
\hline SDSSJ214009.01-064403.9 & $3.8 \pm 1.4$ & $7.0 \pm 2.5$ & $1.9 \pm 0.8$ & $3.5 \pm 1.4$ & $1.9 \pm 1.2$ & $3.5 \pm 2.2$ & $7.6 \mathrm{u}$ & $\cdots$ \\
\hline SDSSJ220119.62-083911.6 & & & & & & & & \\
\hline SDSSJ222753.07-092951.7 & $5.7 \pm 2.6$ & $13.1 \pm 5.7$ & $1.4 \pm 1.3$ & $3.2 \pm 2.9$ & $4.3 \pm 2.7$ & $9.9 \pm 6$ & $2.7 \mathrm{u}$ & $6.8 \mathrm{u}^{a}$ \\
\hline SDSSJ233132.83+010620.9 & $6.3 \pm 2.9$ & $46.9 \pm 21.4$ & $2.6 \pm$ & $21.0 \mathrm{u}$ & $3.7 \pm 1.8$ & $25.9 \pm 12$ & $17.8 \mathrm{u}$ & $9.3 \mathrm{u}$ \\
\hline SDSSJ $234657.25+145736.0$ & $1.7 \pm 1.1^{a}$ & $5.8 \pm 3.7^{a}$ & $1.7 \pm 1.1^{a}$ & $5.8 \pm 3.7^{a}$ & $\ldots$ & & $7.5 \pm 3.6$ & $6.7 \mathrm{u}^{a}$ \\
\hline
\end{tabular}

Notes. Columns are as follows: (1) SDSS name. Equivalent width are listed in Cols. (2), (4) and (6) in units of Å. Line fluxes are reported in Cols. (3), (5), (7), (8) and (9) in units of $\mathrm{erg} \mathrm{s}^{-1} \mathrm{~cm}^{-2} .{ }^{(a)}$ Measurement contaminated by narrow absorption lines. Two colons (:) indicate a high uncertainty in the measurement. Letter $u$ marks an upper limit to the measurement.

Table 7. Measurements on $1400 \AA$ A blend region.

\begin{tabular}{|c|c|c|c|c|c|c|c|c|}
\hline \multirow{2}{*}{$\begin{array}{l}\text { SDSS identification } \\
\text { (1) }\end{array}$} & \multicolumn{2}{|c|}{ SIV + OIV] 1400 TOTAL } & \multicolumn{2}{|c|}{ SIV + OIV] $1400_{\mathrm{BC}}$} & \multicolumn{2}{|c|}{ SIV + OIV] $1400_{\text {BLUE }}$} & \multirow{2}{*}{$\begin{array}{c}\text { CII } 1332 \\
F \\
(8)\end{array}$} & \multirow{2}{*}{$\begin{array}{c}\mathrm{OI}+\mathrm{S} \text { II } 1304 \\
F \\
(9)\end{array}$} \\
\hline & $\begin{array}{l}W \\
(2)\end{array}$ & $\begin{array}{c}F \\
(3)\end{array}$ & $\begin{array}{l}W \\
(4)\end{array}$ & $\begin{array}{c}F \\
(5)\end{array}$ & $\begin{array}{l}W \\
\text { (6) }\end{array}$ & $\begin{array}{c}F \\
(7)\end{array}$ & & \\
\hline SDSSJ000807.27-103942.7 & $15.9 \pm 2.1^{a}$ & $40.0 \pm 3.3^{a}$ & $15.9 \pm 2.1^{a}$ & $40.0 \pm 3.3^{a}$ & & & $4.8 \pm 3.6^{a}$ & $1.9 \pm 1.1^{a}$ \\
\hline SDSSJ004241.95+002213.9 & $11.1 \pm 1.8^{a}$ & $38.0 \pm 5.0^{a}$ & $7.7 \pm 1.9^{a}$ & $26.3 \pm 5.9^{a}$ & $3.4 \pm 0.9^{a}$ & $11.7 \pm 2.8^{a}$ & & $\ldots$ \\
\hline SDSSJ021606.41+011509.5 & $19.5 \pm 2.2^{b}$ & $67.6 \pm 3.8^{b}$ & $19.5 \pm 2.2^{b}$ & $67.6 \pm 3.8^{b}$ & & & $11.7 \pm 5.1$ & $10 \pm 3.8$ \\
\hline SDSSJ024154.42-004757.5 & $9.0 \pm 1.5^{a}$ & $25.9 \pm 3.4^{a}$ & $6.4 \pm 1.5^{a}$ & $18.4 \pm 4^{a}$ & $2.6 \pm 1.2$ & $7.5 \pm 3.4$ & $7.9 \pm 2.7^{a}$ & $8.3 \pm 2.1^{a}$ \\
\hline SDSSJ084036.16+235524.7 & $7.1 \pm 1$ & $21.0 \pm 2.1$ & $7.1 \pm 1$ & $21.0 \pm 2.1$ & & & $5.4 \pm 2.8^{a}$ & $3.9 \pm 1.8$ \\
\hline SDSSJ101822.96+203558.6 & $9.1 \pm 1.3^{a}$ & $39.3 \pm 3.8^{a}$ & $5.3 \pm 1.3^{a}$ & $23.0 \pm 5^{a}$ & $3.8 \pm 0.7^{a}$ & $16.3 \pm 2.7^{a}$ & $5.3 \pm 2.1^{a}$ & $11.4 \pm 3.5^{a}$ \\
\hline SDSSJ103527.40+445435.6 & $9.1 \pm 1.7^{a}$ & $26.4 \pm 4.2^{a}$ & $6.8 \pm 2.1^{a}$ & $19.8 \pm 5.7^{a}$ & $2.3 \pm 0.8^{a}$ & $6.6 \pm 2.2^{a}$ & $2.6 \mathrm{u}$ & $4 \mathrm{u}$ \\
\hline SDSS105806.16+600826.9 & $12.1 \pm 1.8$ & $49.7 \pm 5.4$ & $8 \pm 1.7$ & $33.0 \pm 6$ & $4.1 \pm 1.2$ & $16.7 \pm 4.7$ & $14.3 \pm 4.7$ & $8 \pm 2.1$ \\
\hline SDSSJ110022.53+484012.6 & $12.8 \pm 1.7$ & $41.8 \pm 3.8$ & $5.7 \pm 1.1$ & $22.1 \pm 3.6$ & $7.1 \pm 1.3$ & $19.7 \pm 3$ & $12.8 \pm 6.1$ & $28 \pm 11.9$ \\
\hline SDSSJ125659.79-033813.8 & $21.0 \pm 3.3^{a}$ & $55.2 \pm 6.8^{a}$ & $15.8 \pm 10.0^{a}$ & $41.7 \pm 26.1^{a}$ & $5.2 \pm 2.9$ & $13.5 \pm 7.3$ & $14.9 \pm 10.8^{a}$ & $27.6 \pm 11.1^{a}$ \\
\hline SDSSJ131132.92+052751.2 & $5.7:^{b}$ & $10.7:^{b}$ & $5.7:^{b}$ & $10.7:^{b}$ & & & & \\
\hline SDSSJ143525.31+400112.2 & $7.2 \pm 1.9^{a}$ & $70.2 \pm 17.4^{a}$ & $5.4 \pm 2.4^{a}$ & $52.2 \pm 22.9^{a}$ & $1.8 \pm 1.7^{a}$ & $18.0 \pm 16.7^{a}$ & $14.2 \pm 10.2$ & $23.4 \pm 18.6^{a}$ \\
\hline SDSSJ144412.37+582636.9 & $12.7 \pm 3.7^{b}$ & $14.7 \pm 4.0^{b}$ & $12.7 \pm 3.7^{b}$ & $14.7 \pm 4^{b}$ & & & $\ldots$ & 20.1 .10 .6 \\
\hline SDSSJ151258.36+352533.2 & $6.4 \pm 1.2^{a}$ & $28.5 \pm 4.7^{a}$ & $4.9 \pm 1.5^{a}$ & $21.8 \pm 6.2^{a}$ & $1.5 \pm 0.7^{a}$ & $6.7 \pm 3.2^{a}$ & & $12.4 \pm 4.3^{a}$ \\
\hline SDSSJ214009.01-064403.9 & $20.5 \pm 2.9^{b}$ & $39.1 \pm 3.8^{b}$ & $18 \pm 3.2$ & $34.3 \pm 5.1$ & $2.5 \pm 1.8^{b}$ & $4.8 \pm 3.4^{b}$ & $3.7 \pm 2.1^{b}$ & $8.7 \pm 2.8$ \\
\hline SDSSJ220119.62-083911.6 & $13.4 \pm 6.6^{b}$ & $71.7 \pm 34.4^{b}$ & $13.4 \pm 6.6^{b}$ & $71.7 \pm 34.4^{b}$ & $\ldots$ & $\ldots$ & $\ldots$ & $\ldots$ \\
\hline SDSSJ222753.07-092951.7 & $10.3 \pm 1.9^{a}$ & $28.2 \pm 4.4^{a}$ & $10.3 \pm 1.9^{a}$ & $28.2 \pm 4.4^{a}$ & $\ldots$ & $\ldots$ & $\ldots$ & $\ldots$ \\
\hline SDSSJ233132.83+010620.9 & & & & & & & & $\ldots$ \\
\hline SDSSJ234657.25+145736.0 & $10.3 \pm 1.6^{a}$ & $41.7 \pm 4.8^{a}$ & $9.8 \pm 1.4^{a}$ & $39.7 \pm 3.9^{a}$ & $0.5:^{a}$ & $2.0:^{a}$ & $20.5 \pm 4.1^{a}$ & $\ldots$ \\
\hline
\end{tabular}

Notes. Columns are as follows: (1) SDSS name. Columns (3), (5), (7), (8) and (9) list line fluxes in units of erg s $\mathrm{cm}^{-2}$. Columns (2), (4) and (6) list the equivalent width in $\AA .{ }^{(a)}$ Measurement contaminated by narrow absorption lines. ${ }^{(b)}$ Measurement contaminated by broad absorption lines. Two colons (:) indicates a high uncertainty in the measurement. Letter $u$ marks an upper limit to the measurement.

We modeled these individual features with single Gaussians, without using a model template emission. On the other hand, the FeIII emission has a most important role in xA sources. It was modeled using the scaled and broadened template of Vestergaard \& Wilkes (2001) based on I Zw 1. However, it is not enough to reproduce the observed FeIII emission. We added two extra emission component at 1914 and $2080 \AA$ (modeled by two Gaussians), to account for the line of FeIII UV \#34 line that may be enhanced by $\operatorname{Ly} \alpha$ fluorescence, and for the broad hump at $2080 \AA$ A, mainly ascribed to the blended features of FeIII UV \#48. Most relevant FeII and FeIII features between 1700 and $2100 \AA$ are individually discussed Appendix C.1.

Table 8 reports the equivalent widths and the fluxes of the most important UV FeII multiplet \#191 (FeII $1785 \AA$ ) and the ones of the total FeII emission, that is the sum of the FeII features described above: at 1715,1785 and $2020 \AA$. Table 9 reports equivalent widths and fluxes of the most important individual features ascribed to FeIII (1914 and $2080 \AA$ ) and the total FeIII emission: the sum of the individual features and the template contribution in the full range. It is interesting to note that FeIII emission is almost always stronger than FeII, by a factor of $\sim 2-3$. The strong FeIII emission will be discussed in Sect. 6.4 and Appendix C.2.

\subsection{The origin of the CIVג1549 line broadening}

The GTC-xA sample shows a very tight correlation between FWHM and $c\left(\frac{1}{2}\right)$ of the total CIV $\lambda 1549$ profile (Fig. 3). The best fitting to our new sample is $c\left(\frac{1}{2}\right) \approx(-0.613 \pm 0.081) \cdot F W H M+$ $(1893 \pm 545) \mathrm{km} \mathrm{s}^{-1}$. This relation is consistent within uncertainties with the one found by S17 involving Pop. A sources. The range of blueshifts is from $\approx-1000$ to $-5000 \mathrm{~km} \mathrm{~s}^{-1}$ in both cases; 
Table 8. Flux and equivalent width of FeII contribution.

\begin{tabular}{ccccc}
\hline \hline \multirow{2}{*}{ SDSS identification } & \multicolumn{2}{c}{ FeII $\lambda 1785$} & \multicolumn{2}{c}{ FeII TOTAL } \\
\cline { 2 - 5 }$(1)$ & $W$ & $F$ & $W$ & $F$ \\
& $(2)$ & $(3)$ & $(4)$ & $(5)$ \\
\hline SDSSJ000807.27-103942.7 & $3.5 \pm 0.7$ & $4.4 \pm 0.7$ & $11.2 \pm 2.8$ & $14.0 \pm 3.2$ \\
SDSSJ004241.95+002213.9 & $2.1 \pm 0.6$ & $5.7 \pm 1.4$ & $6.2 \pm 2.0$ & $16.7 \pm 5.2$ \\
SDSSJ021606.41+011509.5 & $2.5 \pm 0.5$ & $7.0 \pm 1.1$ & $9.7 \pm 2.0$ & $29.3 \pm 5.1$ \\
SDSSJ024154.42-004757.5 & $1.5 \pm 0.2$ & $3.1 \pm 0.4$ & $6.5 \pm 1.1$ & $12.4 \pm 1.8$ \\
SDSSJ084036.16+235524.7 & $1.1 \pm 0.3$ & $2.2 \pm 0.6$ & $7.9 \pm 1.9$ & $16.0 \pm 3.5$ \\
SDSSJ101822.96+203558.6 & $1.7 \pm 0.6$ & $5.5 \pm 1.8$ & $6.8 \pm 1.9$ & $21.0 \pm 5.5$ \\
SDSSJ103527.40+445435.6 & $2.7 \pm 0.6$ & $6.6 \pm 1.2$ & $4.8 \pm 1.1$ & $12.1 \pm 2.5$ \\
SDSS105806.16+600826.9 & $0.4:$ & $1.0:$ & $2.5 \pm 0.8$ & $7.0 \pm 2.2$ \\
SDSSJ110022.53+484012.6 & $1.3 \pm 0.4$ & $3.2 \pm 1.0$ & $3.4 \pm 1.2$ & $8.7 \pm 3.1$ \\
SDSSJ125659.79-033813.8 & $\ldots$ & $\ldots$ & $9.9 \pm 4.3$ & $16.0 \pm 6.7$ \\
SDSSJ131132.92+052751.2 & $1.0 \pm 0.4$ & $2.9 \pm 1.0$ & $2.3 \pm 0.7$ & $6.4 \pm 1.9$ \\
SDSSJ143525.31+400112.2 & $1.1 \pm 0.3$ & $7.4 \pm 1.6$ & $3.8 \pm 1.0$ & $23.6 \pm 5.4$ \\
SDSSJ144412.37+582636.9 & $\ldots$ & $\ldots$ & $5.5 \pm 2.2$ & $5.0 \pm 1.9$ \\
SDSSJ151258.36+352533.2 & $1.9 \pm 0.4$ & $5.3 \pm 1.0$ & $3.8 \pm 0.8$ & $11.0 \pm 1.9$ \\
SDSSJ214009.01-064403.9 & $0.7 \pm 0.3$ & $1.2 \pm 0.5$ & $6.5 \pm 1.5$ & $11.7 \pm 2.4$ \\
SDSSJ220119.62-083911.6 & $\ldots$ & $\ldots$ & $1.1 \pm 0.5$ & $3.8 \pm 1.8$ \\
SDSSJ222753.07-092951.7 & $0.2 \pm 0.1$ & $0.4 \pm 0.2$ & $5.3 \pm 1.3$ & $10.5 \pm 2.3$ \\
SDSSJ233132.83+010620.9 & $1.3 \pm 0.9$ & $9.6 \pm 6.7$ & $5.5 \pm 2.9$ & $35.5 \pm 18.0$ \\
SDSSJ234657.25+145736.0 & $\ldots$ & $\ldots$ & $1.3 \pm 0.5$ & $4.5 \pm 1.5$ \\
\hline
\end{tabular}

Notes. Columns are as follows: (1) SDSS name. (2) and (4) report the equivalent width in units of $\AA$. (3) and (5) correspond to the flux in units of $\mathrm{erg} \mathrm{s}^{-1} \mathrm{~cm}^{-2}$.

however, the distribution of the GTC-xA is weighted in favor of larger shifts and broader FWHM with respect to the one of S17. As noted by S17, the correlation of Fig. 3 justify the decomposition of CIV $\lambda 1549$ profile into the BC and a blueshifted excess, which becomes the dominant source of broadening in the case of the largest FWHM.

\subsection{AlIII 1860 as a high redshift virial estimator}

Identification of a UV virial estimator for high redshift quasars $(z \gtrsim 2)$ has been an important quest for several years (e.g., review by Marziani et al. 2017b). Once $\mathrm{H} \beta$ is lost to ground based spectroscopy, MgII $\lambda 2800$ can be exploited up to $z \sim 2.0$ (Trakhtenbrot \& Netzer 2012; Shen \& Liu 2012; Marziani et al. 2013). CIV $\lambda 1549$ is the obvious candidate for quasars beyond $z \sim 2.0$, but the CIV line profiles often show large blue shifts and or asymmetries whose amplitude is a function of source location along the 4DE1 sequence and also of luminosity (S17). This implies that $M_{\mathrm{BH}}$ estimation using FWHM CIV can be uncertain by a large factor (Sulentic et al. 2007; Netzer et al. 2007; Mejía-Restrepo et al. 2018). There have been several recent attempts to account for the bias associated with the nonvirial broadening of CIV $\lambda 1549$ (Park et al. 2013; Matsuoka et al. 2013; Brotherton et al. 2015; Denney et al. 2016; Coatman et al. 2017), however no correction yields safe $M_{\mathrm{BH}}$ estimates especially if Population A and B sources are not considered separately. We focus here on extreme Pop. A (xA) sources and attempt to use AlIII $\lambda 1860$ as an $\mathrm{H} \beta$ virial surrogate. The first composites of low $z$ FOS sources suggested that AlIII $\lambda 1860$ becomes more prominent at the extreme Pop. A end of the 4DE1 sequence (Bachev et al. 2004). The AlIII $\lambda 1860$ line shows a FWHM similar to the one of $\mathrm{H} \beta$, which suggests that both lines are emitted in similar dynamical condition (Negrete et al. 2013). Analysis of VLT ISAAC + FORS spectra of high- $z$ Pop. A sources (Marziani et al. 2017b) show also that FWHM AlIII $\lambda 1860$ agrees well with FWHM H $\beta$. Previously, the emission lines associated with the $1900 \AA$ blend such as AlIII $\lambda 1860$ or CIII] $\lambda 1909$ were avoided due to severe blending (Shen \& Liu 2012), and the $1900 \AA$ blend remains poorly studied to date. Our earlier work motivated us to identify xA source candidates and

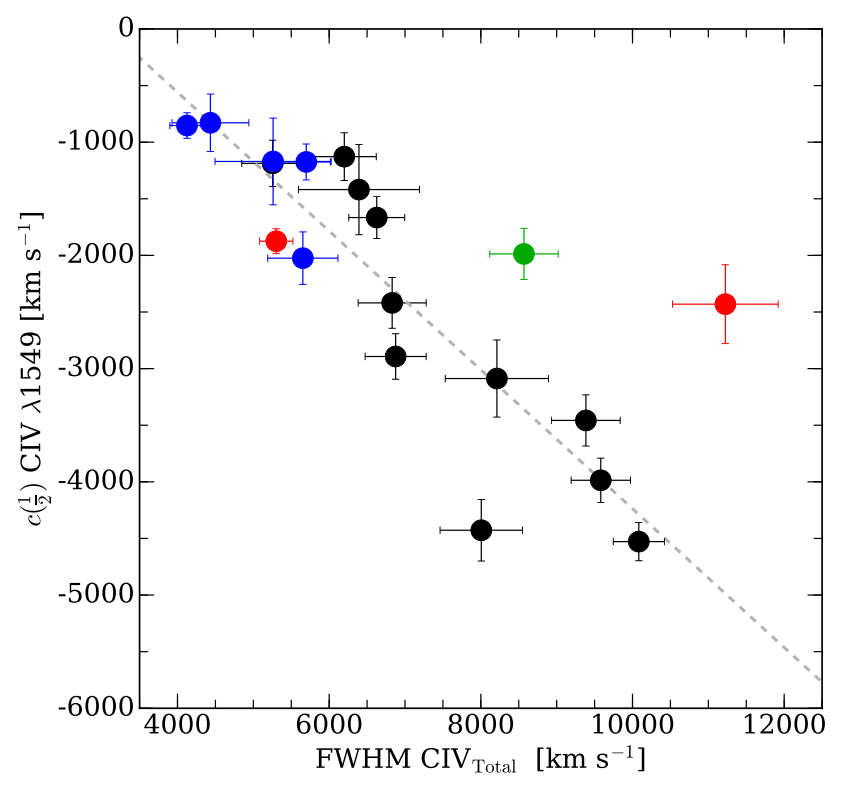

Fig. 3. Relation between the $c\left(\frac{1}{2}\right)$ and the FWHM of the total CIV $\lambda 1549$ emission. Dashed gray line corresponds to the best fit using an orthogonal method. Black, blue, green and red filled circles correspond respectively to normal, BAL, mini-BAL and RL quasars for the GTC-xA sample.

to obtain high S/N spectra of CIV $\lambda 1549$ and the $1900 \AA$ A blend. In the majority of the XA candidates observed so far the AlIII $\lambda 1860$ profile is well defined and is not strongly affected by absorption features (see Sect. 5.3).

The left panel of Fig. 4 shows a comparison between FWHM CIV $\lambda 1549$ and FWHM AlIII $\lambda 1860$ for the xA sample. The ratio between both FWHMs as a function of the FWHM AlIII $\lambda 1860$ is shown in the bottom part of the panel. Coatman et al. (2017) proposed an empirical correction to the CIV profile to obtain the contribution associated with the virial component. Its factor is based on a measure of the CIV blueshift analog of the $c\left(\frac{1}{2}\right)$ we measure. We then applied a correction to the FWHM CIV $\lambda 1549$ proportional to $c\left(\frac{1}{2}\right)$ CIV $\lambda 1549$ to correct for the excess broadening due to the blueshifted component. The result is shown in the right panel of Fig. 4. All CIV $\lambda 1549$ measurements have FWHM larger than FWHM AlIII $\lambda 1860$ before correction. Sources with FWHM AlIII $\lambda 1860 \approx 3000 \mathrm{~km} \mathrm{~s}^{-1}$ show a bias factor of $\approx 2.5-3$ in CIV FWHM, implying almost an order-of-magnitude excess in $M_{\mathrm{BH}}$ estimates. After correction, even if the bias is almost zeroed, we find no significant improvement over the uncorrected panel. This is especially true when we examine the spectra of the 4-6 sources with largest differences with FWHM AlIII $\lambda 1860$. CIV, in most of them, is affected by absorptions. Most of the highest confidence measures involve the cluster of sources in the lower left corner of the figure, which can be argued to be the highest confidence $\mathrm{xA}$ sources. However the sample is too small to allow us to go further. In a forthcoming paper comparing FWHM $\mathrm{H} \beta$ and FWHM AlIII $\lambda 1860$ measures for our low and higher $z$ samples (Marziani et al., in prep.), we find a clear correlation for Pop. A sources. FWHM AlIII $\lambda 1860$ provides the safest equivalent of FWHM H $\beta$ for virial estimation in Pop. A sources.

\subsection{Prevalence and properties of absorbed systems}

The CIV $\lambda 1549$ emission line profile of our GTC-xA sources frequently shows absorptions: only in 9 cases the profile is not contaminated by deep broad absorptions and in only 5 cases 
Table 9. Flux and equivalent width of FeIII contribution.

\begin{tabular}{|c|c|c|c|c|c|c|}
\hline \multirow{2}{*}{$\begin{array}{l}\text { SDSS identification } \\
\text { (1) }\end{array}$} & \multicolumn{2}{|c|}{ Fe III $\lambda 1914$} & \multicolumn{2}{|c|}{ Fe III $\lambda 2080$} & \multicolumn{2}{|c|}{ Fe III TOTAL } \\
\hline & $\begin{array}{l}W \\
\text { (2) }\end{array}$ & $\begin{array}{c}F \\
\text { (3) }\end{array}$ & $\begin{array}{l}W \\
\text { (4) }\end{array}$ & $\begin{array}{c}F \\
(5)\end{array}$ & $\begin{array}{l}W \\
\text { (6) }\end{array}$ & $\begin{array}{c}F \\
(7)\end{array}$ \\
\hline SDSSJ000807.27-103942.7 & $1.9 \pm 0.7$ & $1.9 \pm 0.7$ & $7.5 \pm 0.8$ & $5.9 \pm 0.6$ & $9.9 \pm 2.0$ & $17.1 \pm 3.0$ \\
\hline SDSSJ004241.95+002213.9 & $2.9 \pm 0.8$ & $7.1 \pm 2.0$ & $6.4 \pm 1.1$ & $13.8 \pm 2.5$ & $4.5 \pm 1.3$ & $31.6 \pm 8.8$ \\
\hline SDSSJ021606.41+011509.5 & $5.8 \pm 0.9$ & $16.2 \pm 2.4$ & $6.6 \pm 0.7$ & $17.7 \pm 1.9$ & $25.1 \pm 3.4$ & $103.9 \pm 9.8$ \\
\hline SDSSJ024154.42-004757.5 & $2.3 \pm 1.0$ & $3.8 \pm 1.7$ & $0.1 \mathrm{u}$ & $0.2 \mathrm{u}$ & $12.6 \pm 3.9$ & $24.6 \pm 7.2$ \\
\hline SDSSJ084036.16+235524.7 & $3.4 \pm 1.8$ & $6.1 \pm 3.1$ & $\ldots$ & $\ldots$ & $23.0 \pm 3.2$ & $44.5 \pm 4.3$ \\
\hline SDSSJ101822.96+203558.6 & $4.7 \pm 0.9$ & $13.7 \pm 2.7$ & $6.8 \pm 1.3$ & $17.2 \pm 3.2$ & $11.8 \pm 2.7$ & $63.9 \pm 13.1$ \\
\hline SDSSJ103527.40+445435.6 & $5.8 \pm 0.7$ & $13.1 \pm 1.7$ & $9.0 \pm 0.8$ & $18.3 \pm 1.6$ & $3.1 \pm 0.6$ & $38.2 \pm 6.7$ \\
\hline SDSS105806.16+600826.9 & $3.5 \pm 0.5$ & $8.0 \pm 1.1$ & $1.8 \pm 0.9$ & $3.8 \pm 1.8$ & $12.9 \pm 2.7$ & $40.6 \pm 7.3$ \\
\hline SDSSJ110022.53+484012.6 & $0.9 \pm 0.6$ & $2.0 \pm 1.4$ & $3.2 \pm 1.0$ & $6.1 \pm 1.9$ & $5.6 \pm 2.5$ & $20.0 \pm 8.6$ \\
\hline SDSSJ125659.79-033813.8 & $2.6 \pm 2.3$ & $4.3 \pm 3.8$ & $6.5 \pm 2.7$ & $9.0 \pm 3.8$ & $7.8 \pm 6.0$ & $25.7 \pm 19.5$ \\
\hline SDSSJ131132.92+052751.2 & $3.1 \pm 0.4$ & $8.6 \pm 1.0$ & $3.1 \pm 0.5$ & $8.1 \pm 1.2$ & $4.4 \pm 0.9$ & $28.7 \pm 5.0$ \\
\hline SDSSJ143525.31+400112.2 & $3.2 \pm 0.7$ & $19 \pm 4.4$ & $2.1 \pm 0.6$ & $11.0 \pm 3.2$ & $8.7 \pm 2.0$ & $79.4 \pm 16.1$ \\
\hline SDSSJ144412.37+582636.9 & $3.6 \pm 1.3$ & $3.2 \pm 1.1$ & $4.0 \pm 2.1$ & $3.2 \pm 1.7$ & $4.3 \pm 3.3$ & $10.2 \pm 7.9$ \\
\hline SDSSJ151258.36+352533.2 & $3.0 \pm 0.6$ & $7.3 \pm 1.5$ & $2.1 \pm 0.6$ & $4.5 \pm 1.3$ & $6.7 \pm 1.7$ & $27.2 \pm 6.3$ \\
\hline SDSSJ214009.01-064403.9 & $3.7 \pm 0.8$ & $6.5 \pm 1.4$ & $5.3 \pm 0.8$ & $9.0 \pm 1.4$ & $4.3 \pm 1.2$ & $21.9 \pm 5.9$ \\
\hline SDSSJ220119.62-083911.6 & $2.0 \pm 0.6$ & $7.6 \pm 2.5$ & $1.1 \pm 0.7$ & $3.6 \pm 2.5$ & $8.0 \pm 2.6$ & $41.2 \pm 13.0$ \\
\hline SDSSJ222753.07-092951.7 & $1.0 \pm 0.5$ & $1.8 \pm 0.9$ & $7.1 \pm 1.1$ & $10.4 \pm 1.6$ & $8.0 \pm 2.1$ & $25.4 \pm 6.1$ \\
\hline SDSSJ233132.83+010620.9 & $1.3 \pm 0.3$ & $8.7 \pm 2.3$ & $3.3 \pm 0.8$ & $20.5 \pm 4.9$ & $4.4 \pm 2.2$ & $57.8 \pm 28.8$ \\
\hline SDSSJ234657.25+145736.0 & $2.1 \pm 0.3$ & $6.0 \pm 1.0$ & $1.1 \pm 0.8$ & $2.7 \pm 2.1$ & $8.6 \pm 2.4$ & $32.9 \pm 8.7$ \\
\hline
\end{tabular}

Notes. Columns are as follows: (1) SDSS name. Columns (2), (4) and (6) list the equivalent width in units of A. Columns (3), (5) and (7) correspond to the flux in units of $\mathrm{erg} \mathrm{s}^{-1} \mathrm{~cm}^{-2}$.
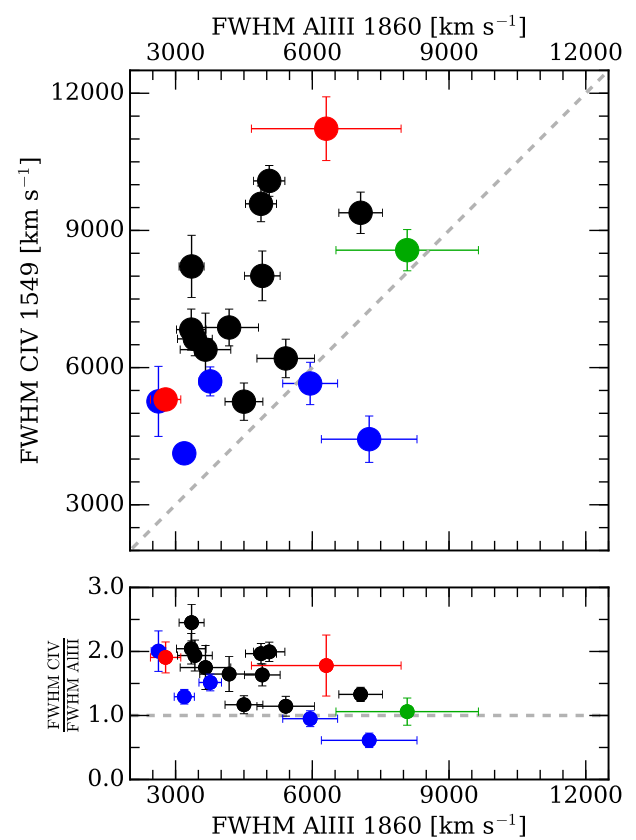
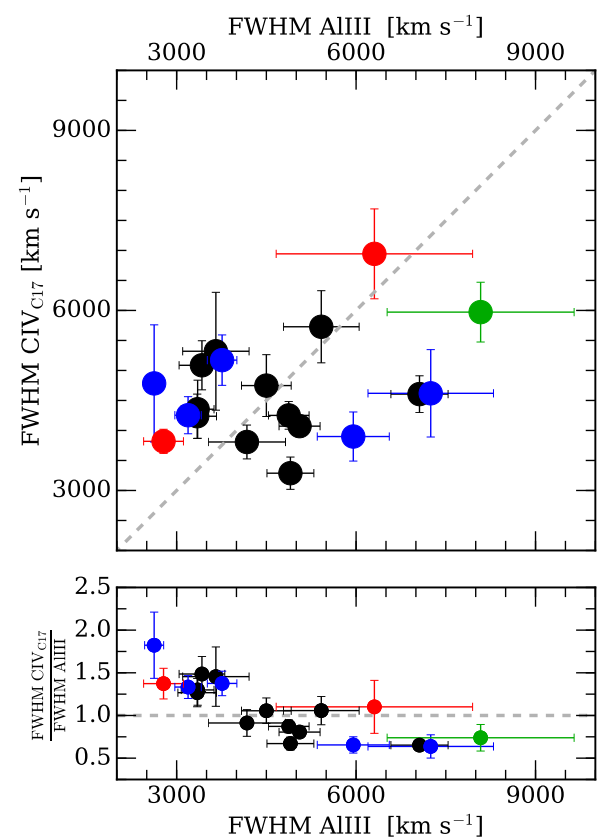

Fig. 4. Relation between the FWHM AlIII $\lambda 1860$ and FWHM CIV $\lambda 1549$ before (left) and after (right) Coatman et al. (2017) correction. Dashed gray line corresponds to the $1: 1$ line. Bottom panels: ratio between the FWHM CIV $\lambda 1549$ and AlIII $\lambda 1860$. Colors are the same as the Figs. 2 and 3. the profile is not affected by any narrow or broad absorption. In order to ascertain whether our sample suffers from unusually strong line absorption compared to a general quasar population at $2.0 \lesssim z \lesssim 2.9$, we considered estimates of the absorption index (AI) and Balnicity index (BI) as defined in Scaringi et al. (2009). The AI (Hall et al. 2002) includes semibroad absorptions continuously detected over a range of at least $1000 \mathrm{~km} \mathrm{~s}^{-1}$, from 0 to $-29000 \mathrm{~km} \mathrm{~s}^{-1}$; the BI (Weymann et al. 1991) measures contiguous absorption features over $2000 \mathrm{~km} \mathrm{~s}^{-1}$ in the range of -25000 to $-3000 \mathrm{~km} \mathrm{~s}^{-1}$, and is intended to identify quasars with broad through features almost certainly not associated with external absorbers. Table 10 reports the AI and BI measures for our sample. Large CIV $\lambda 1549$ BI absolute values $\left(\lesssim-1000 \mathrm{~km} \mathrm{~s}^{-1}\right)$ are associated with Pop. A and with "strong"
BALs with large equivalent widths and high up to terminal velocity, while mini-BALs are also found at low- $z$ among Pop. B and RL objects (Sulentic et al. 2006a). Trump et al. (2006) provide both AI and BI measurements obtained from a SDSS DR3 catalog containing 5719 quasars in redshift range $z \sim 2-2.6$ (Schneider et al. 2005). There are 542 sources with CIV $\lambda 1549$ BI $<0$ suggesting $\approx 9.5 \%$ for "strong" BAL sources, consistent with conventionally quoted prevalence values for BAL QSOs. In the GTC-xA sample, 5 over 19 sources show CIV $\lambda 1549 \mathrm{BI}<, 0$. If we also include one source with CIV $\mathrm{BI}=0$ (SDSSJ144412.37+582636.9), but with strong absorption in the blue side of Sirv $\lambda 1397+$ OIV blend at $1400 \AA$ and $\mathrm{BI} \ll 0$ (see Fig. A.13), we obtain a prevalence of BAL QSOs around $25-30 \%$. AI is less than 0 in 10 out of 19 sources of the 
Table 10. Balnicity and Absorption indices.

\begin{tabular}{lcc}
\hline \hline \multicolumn{1}{c}{ SDSS identification } & & \\
\hline SDSSJ000807.27-103942.7 & $\begin{array}{c}\text { BI } \\
(2)\end{array}$ & $\begin{array}{c}\text { AI } \\
(3)\end{array}$ \\
\hline SDSSJ004241.95+002213.9 & 0 & 0 \\
SDSSJ021606.41+011509.5 & 0 & 0 \\
SDSSJ024154.42-004757.5 & 0 & -2575 \\
SDSSJ084036.16+235524.7 & 0 & 0 \\
SDSSJ101822.96+203558.6 & 0 & -210 \\
SDSSJ103527.40+445435.6 & 0 & 0 \\
SDSS105806.16+600826.9 & 0 & -160 \\
SDSSJ110022.53+484012.6 & 0 & 0 \\
SDSSJ125659.79-033813.8 & -720 & 0 \\
SDSSJ131132.92+052751.2 & -2440 & -970 \\
SDSSJ143525.31+400112.2 & 0 & 0 \\
SDSSJ144412.37+582636.9 & 0 & -770 \\
SDSSJ151258.36+352533.2 & 0 & -270 \\
SDSSJ214009.01-064403.9 & -2530 & -3620 \\
SDSSJ220119.62-083911.6 & -5800 & -7460 \\
SDSSJ222753.07-092951.7 & 0 & 0 \\
SDSSJ233132.83+010620.9 & 0 & 0 \\
SDSSJ234657.25+145736.0 & 0 & -240 \\
\hline
\end{tabular}

Notes. Columns are as follows: (1) SDSS name. (2) Balnicity index in units of $\mathrm{km} \mathrm{s}^{-1}$. (3) Absorption index in units of $\mathrm{km} \mathrm{s}^{-1}$.

GTC-xA sample; about 30\% of Trump et al. (2006) satisfy the same condition. If one considers that about $1 / 2$ of SDSS quasars are Pop. A quasars (Zamfir et al. 2010), the prevalence of strong BAL sources should be doubled to $\sim 20 \%$. We can therefore conclude that the GTC-xA sample does not show an unusually large fraction of intrinsically absorbed systems, possibly at a prevalence somewhat higher than the general quasar population.

\section{Discussion}

\subsection{Equivalent width distributions}

Figure 5 shows a comparison between the equivalent width distributions of AlIII $\lambda 1860$, SiIII] $\lambda 1892$, CIII] $\lambda 1909$ and CIV $\lambda 1549$ for the GTC-xA sources and the three control samples: the low$z$ (FOS-A and FOS-xA) and the high- $z$ (S14-A) samples. The AlIII $\lambda 1860$ line of the GTC-xA shows an enhancement relative to the other samples (upper left panel). The AlIII values distribution for the GTC-xA sources is significantly different more than $6 \sigma$ respect to the ones observed in FOS-A and S14-A, according to a T-test ( $6.7 \sigma$ and $6.1 \sigma$, respectively). In fact, a KolmogorovSmirnov (KS) test gives a very low probability of being equal population between GTC-xA, and FOS-A and S14-A $\left(8 \times 10^{-6}\right.$ and $5 \times 10^{-6}$, respectively). Meanwhile, there is no statistically significant difference between the two Pop. A samples (FOS-A and S14-A) or the two Pop. xA (GTC-xA and FOS-xA) samples.

The SiIII] $\lambda 1892$ equivalent width (upper right panel) covers a wide range of values with no statistical significant difference between the samples. Only at a level of $2 \sigma$, the GTC-xA shows slightly higher values of $W$ SiIII] $\lambda 1892$ than FOS-A sample. But neither the T-test nor the KS test yield a significant probability of being different populations.

Instead, in the GTC-xA sample the $W$ CIII] distribution (bottom left panel) appears to be markedly weaker than a Pop. A. Both T-test and KS-test give significant differences at $6.5 \sigma$ and $5.2 \sigma$ level for the GTC-xA sample to be a different population respect to FOS-A and S14-A respectively. The probability according to the KS test is very low $\left(3.2 \times 10^{-6}\right.$ and $1 \times 10^{-4}$, respectively). In xA sources CIII] $\lambda 1909$ is weak and blended with the FeIII contribution, to the point of not being clearly detectable in several cases. On the other hand, in the Pop. A samples (FOS-A and S14-A) this ion is perfectly appreciable by naked eye and specially prominent in Pop. B sources (e.g., Bachev et al. 2004; Kuraszkiewicz et al. 2004)

Bottom right panel of Fig. 5 shows a comparison between the equivalent width distributions of CIV $\lambda 1549$ for $\mathrm{xA}$ and Pop. A sources. xA values are the ones with the lowest $W$ CIV $\lambda 1549$ among Pop. A sources, with a wide majority of sources distributed below $\sim 40 \AA$. On the converse, the FOS-A and S14-A samples show a much broader distribution, with no evidence of a systematic difference between them, within the limitations of the relatively small sample sizes. The statistical tests gives a significant difference between GTC-xA and FOS-A and S14-A at $5 \sigma$ and $7 \sigma$, and a KS-test a low probability $\left(\sim 10^{-5}\right)$ to be the same population.

These histograms give a first hint to the fact that xA quasars show a different spectroscopic behavior compared with the one shown by samples of the full Population A. Due to there are not any significant differences in the equivalent width distributions of two xA samples (GTC-xA and FOS-xA) and neither between the two Pop. A samples (FOS-A and S14-A), we can consider a sample with $24 \mathrm{xA}$ sources $(19+5)$ and 34 Pop. A sources $(23+11)$. A comparison of the AlIII $\lambda 1860$ equivalent width of the $24 \mathrm{xA}$ respect to 34 Pop. A sources shows a significant difference between them at a level of $7.8 \sigma$ with a low probability to be the same distribution $\left(7 \times 10^{-8}\right)$. There is also a statistically significant difference in the distributions of CIII] and CIV $\lambda 1549$, but in the opposite direction: values of the xA sources are significantly lower than Pop. A $(6.6 \sigma$ and $5 \sigma$ level respectively with probabilities lower than $\left.2 \times 10^{-7}\right)$.

\subsection{Composite spectra at low and high-z}

With the objective of highlight main features observed in the $\mathrm{xA}$ spectrum and make a comparison with control samples, we built a composite spectrum using the full GTC-xA sample. Multicomponent fits and its analysis are shown in Sect. 5.1. At the top level of Fig. 6 is represented the composite GTCXA spectrum. It clearly shows an enhancement of AlIII and SiIII], in contrast with the weakness of CIII], which has mean $W$ CIII] $~ 4 \AA$. Also, FeIII becomes the largest contribution in the red side of the $1900 \AA$ blend, which is also prominent in the FeIII feature detected at $\sim 2080 \AA$. Another noticeable feature is the almost fully blueshifted CIV $\lambda 1549$ profile, which shows a low equivalent width. A blueshift is also observed in SiIV $\lambda 1397+$ OrV] $\lambda 1402$, although in this case the profile is more symmetric due to the presence of strong absorptions in its blue side. As we point out in Sect. 5.4, HeII $\lambda 1640$ is very weak with a flat topped appearance profile. Low-ionization features, CII $\lambda 1335$ and OI $\lambda 1304+$ SiII $\lambda 1306$, are also clearly appreciated.

In order to compare the general behavior of xA sources, we also built composite spectra for two comparison samples: FOS-xA and S14-A samples, middle and bottom level of Fig. 6, respectively. A multicomponent analysis was also applied to composite control samples. The width line of AlIII $\lambda 1860$ in FOS$\mathrm{xA}\left(\sim 2740 \mathrm{~km} \mathrm{~s}^{-1}\right)$ is narrower than GTC-xA $\left(\sim 4500 \mathrm{~km} \mathrm{~s}^{-1}\right)$. The average bolometric luminosity of the FOS-xA sample is $\sim 2.4 \times 10^{45} \mathrm{erg} \mathrm{s}^{-1}$ and $\sim 10^{47} \mathrm{erg} \mathrm{s}^{-1}$ for the GTC-xA, therefore a FWHM change can be associated with the higher luminosity in the GTC-xA sample.

The S14-A composite spectrum shows a comparable luminosity $(\log L \approx 46)$ than the FOS-xA, and a similar redshift that GTC-XA sample. S14-A shows a strong and symmetric 

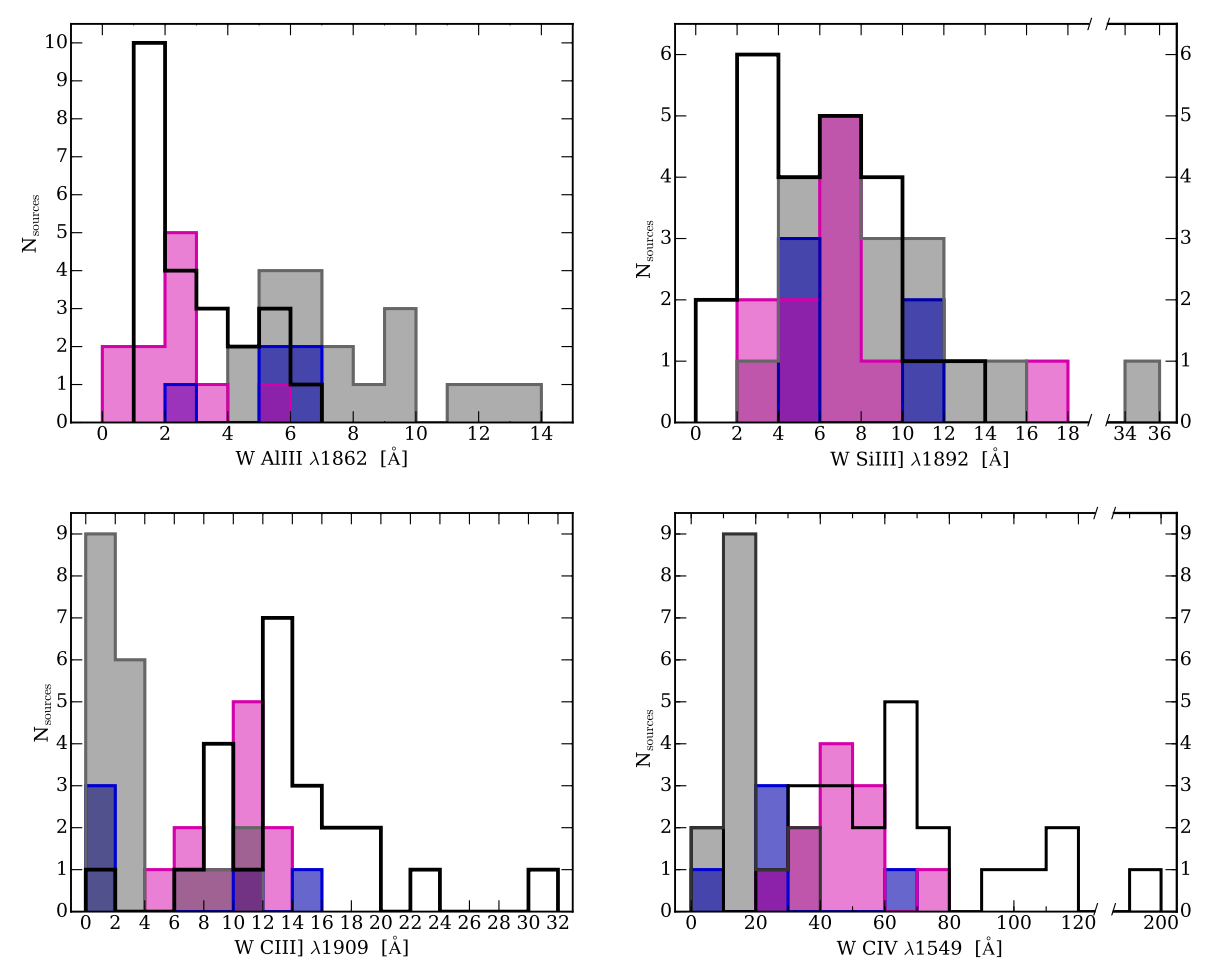

Fig. 5. Rest-frame equivalent width distributions of the AlIII $\lambda 1860$, SiIII] $\lambda 1892$, CIII] $\lambda 1909$ and CIV $\lambda 1549$ for the GTC-xA (gray), FOS-xA (blue), FOS-A (not filled) and S14-A (magenta) samples.

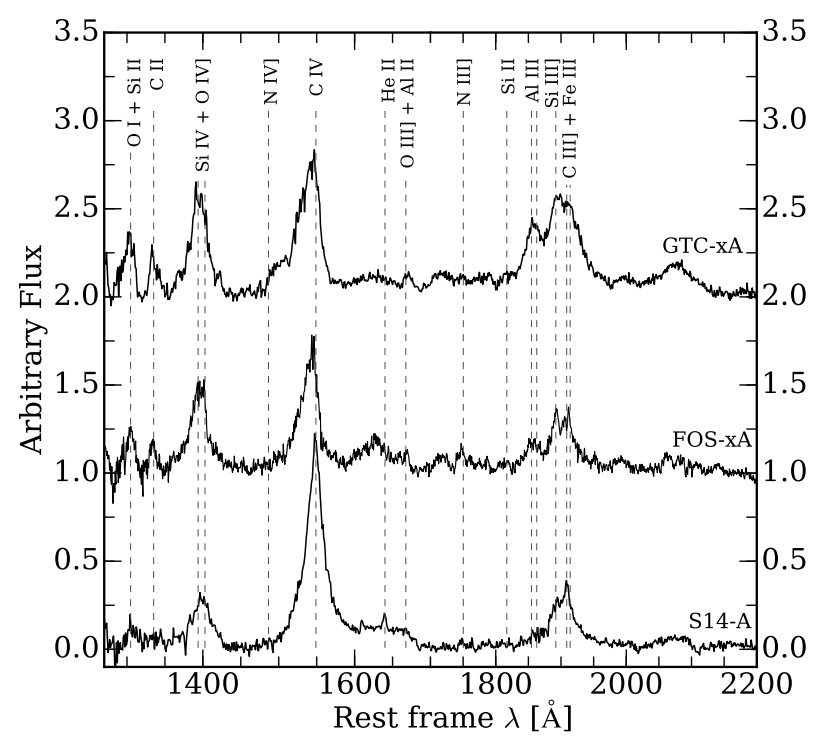

Fig. 6. From top to bottom, rest-frame composite spectra of the GTC$\mathrm{xA}$, FOS-xA, and S14-A. Intensity units are arbitrary flux. The GTC-xA and FOS-xA have been displaced by a constant value for clarity.

CIV $\lambda 1549$ profile with a median equivalent width of $47 \AA$, more than two times large than the median values obtained in xA samples: 14 and $23 \AA$ for GTC-xA and FOS-xA, respectively. Conversely, the $1900 \AA$ blend of S14-A composite spectrum is dominated by $\mathrm{CIII}]$ and SiIII] profiles. The xA samples show a weaker $\mathrm{CIII}$, with median values of equivalent width $2.6 \AA$ for GTC-xA, $1.8 \AA$ for FOS-xA and $11 \AA$ for S14-A. Also they shows a stronger AlIII $\lambda 1860$ that Pop. A samples, with the next median values: $7 \AA$ for the GTCxA, $6 \AA$ for FOS-xA and $2 \AA$ for S14-A. Other notable features of $\mathrm{xA}$ sources include a smaller HeII $\lambda 1640$, as well as a higher (OIV] $\lambda 1402+\operatorname{SiIV} \lambda 1397) / \mathrm{CIV} \lambda 1549_{\mathrm{BC}}$ ratio. Features like OI $\lambda 1304+$ SiII $\lambda 1306$ and CII $\lambda 1335$ are also clearly observed, supporting the inference of a low-ionization degree for $\mathrm{xA}$ objects (see Sect. 6.3). There is a fundamental caveat between GTC-xA and S14-A samples: among the faint sources of S14-A there is no xA sources. A systematic difference in $L / L_{\text {Edd }}$ of $\approx 2$ can be accounted, if the Eddington ratio of GTC-xA sources is $L / L_{\mathrm{Edd}} \approx 1$ and if the S14-A are instead radiating at $L / L_{\mathrm{Edd}} \approx$ 0.3 (Sulentic et al. 2014a), without invoking any effect of luminosity.

\subsection{A low-ionization spectrum with prominent FellI}

The UV spectral range provides several diagnostic ratios that can constrain physical parameters. For instance the CIII] $\lambda 1909 /$ SiIII] $\lambda 1892$ and AlIII $\lambda 1860 /$ SiIII] $\lambda 1892$ ratios are sensitive to hydrogen density, CIV $\lambda 1549 /$ AlIII $\lambda 1860$ and CIV $\lambda 1549 /$ SiIII] $\lambda 1892$ to ionization, and HeII $\lambda 1640 / \operatorname{CIV} \lambda 1549$ and (OIV] $\lambda 1402+$ SiIV $\lambda 1397) / C I V \lambda 1549$ to metallicity (N12, Negrete et al. 2013, 2014). The diagram of Fig. 7 has been computed from an array of CLOUDY 13.05 (Ferland et al. 2013) simulations covering the range in ionization parameter $-4.5 \leq$ $\log U \leq 0$ and density $7 \leq n_{\mathrm{H}} \leq 14 \mathrm{~cm}^{-3}$, with steps of $0.25 \mathrm{dex}$. A total of 551 simulations were carried out assuming a standard AGN continuum appropriate for Pop. A, a column density $N_{\mathrm{c}}=10^{23} \mathrm{~cm}^{-2}$ and a metallicity of $100 Z_{\odot}$. The median flux ratios given by AlIII $\lambda 1860 /$ SiIII] $\lambda 1892$, CIV $\lambda 1549 /$ AlIII $\lambda 1860$, and CIV $\lambda 1549 / \operatorname{SiIII} \lambda \lambda 1892$ converge toward $\log U \approx-3$ and $\log n_{\mathrm{H}} \approx 13\left[\mathrm{~cm}^{-3}\right]$. The suggestion of low-ionization and high density is in agreement with the previous study of two xA sources by N12. Consistently with the inference of high density, CIII] $\lambda 1909$ is usually weak although not entirely negligible with a $\log \mathrm{CIII}] \lambda 1909 / \mathrm{SiIII}] \lambda 1892 \approx-0.42$. Due to the critical electronic density of the CIII] $\lambda 1909$ is $n_{\mathrm{H}}=10^{10} \mathrm{~cm}^{-3}$, this line is expected to be collisionally suppressed with respect to AlIII $\lambda 1860$ and SiIII] $\lambda 1892$, as it is shown by the GTCxA composite spectrum. The strong FeIII contribution observed in the GCT-xA sources also supports the existence of a very 


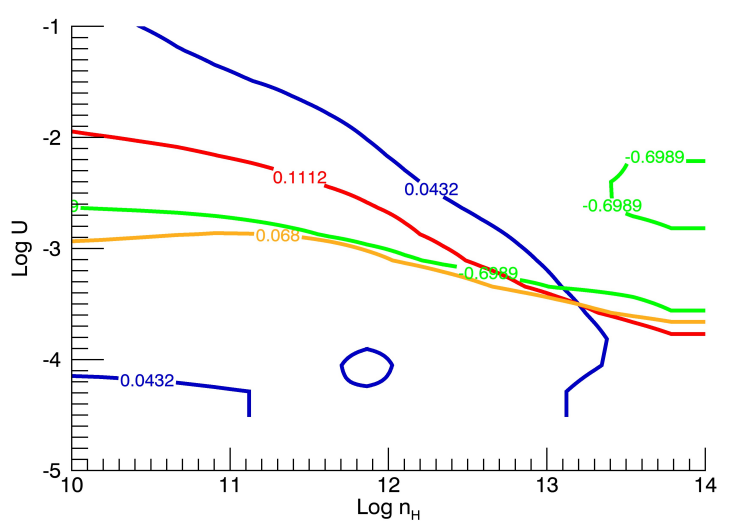

Fig. 7. Isocontours in the plane log of ionization parameter $(U)$ versus log of hydrogen density $\left(n_{\mathrm{H}}\right)$ from CLOUDY simulations for the line intensity ratios AlIII $\lambda 1860 /$ SiIII] $\lambda 1892$ (blue), HeII $\lambda 1640 / C I V \lambda 1549$ (green), CIV $\lambda 1549 /$ AlIII $\lambda 1860$ (yellow), CIV $\lambda 1549 /$ SiIII] $\lambda 1892$ (red) using $Z=100 Z_{\odot}$. Labels report the median values of the intensity ratios for the objects of the GTC-xA sample.

low-ionization spectrum, $\log U \leq-2$. A more detailed analysis of FeIII emission is presented in Appendix C.

The intensity ratios considered above are not strongly sensitive to metallicity except for the HeII $\lambda 1640 / \operatorname{CIV} \lambda 1549$ and the (OIV] $\lambda 1402+\operatorname{SiIV} \lambda 1397) / \operatorname{CIV} \lambda 1549$ ratios. According to CLOUDY simulations, at solar metallicity we expect CIV $\lambda 1549 / \mathrm{HeII} \lambda 1640 \approx 1$ for the low- $U$ and high density solution, which is very far from the value that we derive for the $\mathrm{xA}$ sample ( 0.19). A possible solution is offered by a large increase in metallicity above solar values. The HeII $\lambda 1640 / \operatorname{CIV} \lambda 1549$ is the ratio between a recombination and a collisionally excited line: at high- $Z$, the metals absorb a larger fraction of the ionizing flux, and the He recombination line become weaker. This effect leads to a decrease of the HeII $\lambda 1640 /$ CIV $\lambda 1549$ over the value expected at solar $Z$ (Hamann \& Ferland 1993, 1999). In this case, the $Z$-sensitive HeII $\lambda 1640 / \mathrm{CIV} \lambda 1549$ would be small $(\sim 0.2)$ even at low-ionization. An increment in the metallicity to $Z \approx 100 Z_{\odot}$ gives such low HeII $\lambda 1640 /$ Civ $\lambda 1549$ ratios (Fig. 7). The value $Z \approx 100 Z_{\odot}$ may appear unrealistically high. However, N12 found evidence of high-Z, as well as of possible abundance anomalies. It appears reasonable to assume very high values of $Z$ for $x A$ sources: Pop. A sources at low $-z$ and high $z$ have NV $\lambda 1240 / C I V \lambda 1549$ and (OIV] $\lambda 1402+\operatorname{SiIV} \lambda 1397) / \operatorname{CIV} \lambda 1549$ implying $Z \gtrsim 10 Z_{\odot}$ (Shin et al. 2013, S14). The median ratio SiIV $\lambda 1397 / \operatorname{CIV} \lambda 1549 \sim 1$ may suggest a metallicity significantly higher than 10 times solar for the GTC-xA sample. The high SiIV $\lambda 1397+$ OIV] $\lambda 1402 / \operatorname{CIV} \lambda 1549$ ratio also supports this suggestion. In agreement with the interpretation of Hamann \& Ferland (1999), the equivalent width of HeII $\lambda 1640$ is extremely low, making the line almost undetectable above noise.

We should also consider that our approach may overestimate the $\mathrm{CIV}_{\mathrm{BC}}$ intensity, as it attempts to maximize its contribution attempting to account for the red side of the profile, even when the profile if almost fully blue shifted. There is the possibility that the $\mathrm{CIV}_{\mathrm{BC}}$ is significantly weaker. A slightly lower value of $Z=50 Z_{\odot}$ is again in agreement with the observations assuming an overestimation of the $\operatorname{CIV}_{\mathrm{BC}}$ intensity by factor of two. Therefore, a $Z$ value of several tens $Z_{\odot}$ might be considered as a lower limit if we consider a systematic overestimation of the $\mathrm{CIV}_{\mathrm{BC}}$. It is unlikely that the overestimate might be much larger than a factor of two, as the $\mathrm{CIV}_{\mathrm{BC}}$ is clearly detectable

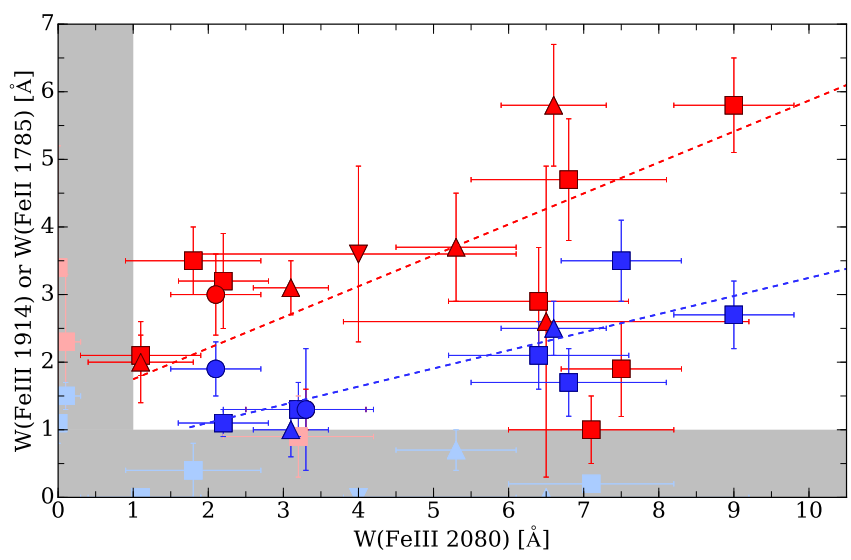

Fig. 8. Relation between intensity of the FeIII feature at $2080 \AA$ with Fe UV $1785 \AA$ (blue) and FeIII 11914 (red). Squares, up and down triangles, and circles mark the normal, BAL, mini-BAL and RL quasars, respectively. The gray shaded area indicates an approximate detection limit. Blue and red lines mark the orthogonal fit for each case.

while HeII $\lambda 1640$ equivalent width is extremely low. More precise inferences require dedicated photoionization computations beyond the scope of this paper.

\subsection{Evidence of $2080 \AA$ enhancement}

Several sources show a significant excess with respect to the template at around $\sim 2080 \AA$, indicating that UV \#48 is selectively enhanced with respect to the "normal" emission in xA sources as represented by Vestergaard \& Wilkes (2001) template. In eight sources the equivalent width of the feature in excess respect to the template is $\mathrm{W}(2080) \gtrsim 5 \AA$. This excess is evident from the spectra, and is even more outstanding because the HILs in the adjacent spectral regions are of low equivalent width. A mock profile built from the transition of multiplet FeIII UV \# 48 between the terms $\mathrm{a}^{5} S-z^{5} P^{0}$, assuming relative intensity for optically thin transitions (Kovačević et al. 2010) accounts for the $2080 \AA$ feature: the mock profile resulting from the lines broadened to $4000 \mathrm{~km} \mathrm{~s}^{-1}$ is a feature at $\approx 2073 \AA$ with total $F W H M \approx 5000 \AA$, consistent with the observations.

Figure 8 shows the equivalent width of the FeIII $\lambda 1914$ and of the FeII UV\#191 multiplet versus the equivalent width of the $2080 \AA$ feature. Only ten sources satisfy the detection limit we imposed at $W \approx 1 \AA$. Strong FeIII $\lambda 2080$ is usually associated with strong FeIII $\lambda 1914$. According to an orthogonal fit taking into account errors in both axis, these two emission lines follow the relation FeIII $1914=(0.46 \pm 0.14) \cdot \mathrm{FeIII}_{2080}+(1.29 \pm 0.55)$, which verified the null hypothesis of a slope different from 0 at $3.3 \sigma$ significant level. There is a clear relation, although the errors associated with FeIII $\lambda 1914$ are high because of the blend with CIII] $\lambda 1909$ and the contribution of the FeIII template. On the another hand, the FeIIUV \#191 is generally rather weak, but the five cases with a clear detection $(W \gtrsim 2 \AA)$ are associated with strong $2080 \AA$ features. An orthogonal fit gives a relation FeII $_{\mathrm{UV}}=(0.27 \pm 0.06) \cdot$ FeIII $\lambda 2080+(0.57 \pm 0.30)$ with a slope different from 0 at a $4 \sigma$ significance level. This relation considers measurements with $W>1.0 \AA$ A of both FeII UV \#191 and FeIII $\lambda 1914$ (Fig. 8). A fluorescence mechanism (described in Appendix C.2) may account for the selective enhancement of the $2080 \AA$ A feature. 


\subsection{Estimates of the accretion parameters}

\subsubsection{Bolometric correction}

There has been no accurate determination of the bolometric correction (hereafter B.C.) specific to xA sources. An attempt has been made to compute the bolometric correction from the model SEDs of MS14. MS14 suggested that the difference in the optical, UV, X range between a typical NLSy1 and an extreme NLSy1 is about $10 \%$ (see their Sect. 6.1.2.3). It is unlikely that the uncertainty in the B.C. is much larger than $\approx 20 \%$ (at $1 \sigma$ confidence level). SDSS quasars have with B.C. $1350=3.5$ and a scatter $\sim 20 \%$ (Richards et al. 2006). SDSS quasars are however an heterogenous sample composed by quasar of different spectral types. On the contrary, the GTC-XA sample is composed by similar sources. If we consider 27 spectra of sample 3 of MS14, that is at redshift in the range 2.0-2.6, the rms averaged between $\approx 1100$ and $2900 \AA$ is $\approx 10 \%$.

Considering that the bulk of the ionizing energy is coming from the big blue bump in correspondence of the Lyman limit, the SED is not significantly different from the one of Mathews \& Ferland (1987). The MS14 SED differs from the reference Mathews \& Ferland (1987) SED only for the slope in the X-ray domain, and yields a B.C.1350 $\approx 5.4$. The B.C. value is consistent with the one of Elvis et al. (1994). The value of Elvis et al. (1994) may perhaps provide a more appropriate comparison, as the sources in that paper implied a detection from the IPC instrument on board the Einstein observatory (Giacconi et al. 1979), that is in the soft X-ray domain. Bolometric luminosities $L_{\mathrm{Bol}}$ of the GTC-xA sources are presented in Table 11. They have been computed through the monochromatic luminosities at $1350 \AA\left(\lambda L_{\lambda}(1350 \AA)\right)$ and a B.C. $=5.4 \pm 0.6$. A more careful estimate would require a detailed analysis of the mid- and far-IR domain, where part of the accretion luminosity is reradiated, and is deferred to an eventual work.

\subsubsection{Black hole mass}

We estimate black hole mass $\left(M_{\mathrm{BH}}\right)$ using the UV CIV scaling relation of Vestergaard \& Peterson (2006, Eq. (7)) applied to FWHM(AlIII) $)^{3}$. The AlIII profile is usually not affected by the strong outflows often observed in the CIV $\lambda 1549$ profile, thus providing a good approximation to the virial motion of the BLR (e.g., Marziani et al. 2017b, see also Sect. 5.8). We adopt $M_{\mathrm{BH}, \mathrm{AlIII}}$ as the virial mass estimator. Second column of Table 11 shows the $M_{\mathrm{BH}}$ estimations. Typical formal uncertainties in the estimation of the masses, through error propagation, are about $25 \%$ taking into account an uncertainty of $10 \%$ in the flux at $1350 \AA$, and including the estimated errors for the FWHM AlIII $\lambda 1860$.

The GTC-xA sample covers a black hole mass range of $8.8 \leq M_{\mathrm{BH}} \leq 9.8 M_{\odot}$, with a median value of $\left\langle M_{\mathrm{BH}}\right\rangle \sim$ $9.2 M_{\odot}$. The GTC-xA sample is covering the high- $M_{\mathrm{BH}}$ counterparts of the FOS-xA sample. Also these values confirm the well-know tendency of larger black hole mass $\left(\sim 10^{9} M_{\odot}\right)$ at high redshift $(z \approx 2$; Shemmer et al. 2004; Netzer et al. 2007; Trakhtenbrot \& Netzer 2012), a redshift-dependent selection effect common to all flux-limited samples (Sulentic et al. 2014a).

$3 \log M_{\mathrm{BH}}(\mathrm{AlIII})=\log \left\{\left[\frac{\mathrm{FWHM}(\mathrm{AlIII})}{1000 \mathrm{kms}^{-1}}\right]^{2}\left[\frac{\lambda L_{\lambda}(1350 \AA)}{10^{44} \mathrm{erg} \mathrm{s}^{-1}}\right]^{0.53}\right\}+(6.73 \pm 0.01)$.
Table 11. Accretion parameters.

\begin{tabular}{cccc}
\hline \hline SDSS identification & $\log L_{\text {Bol }}$ & $\log M_{\text {BH,AlIII }}$ & $\begin{array}{c}L / L_{\text {Edd }} \\
(4)\end{array}$ \\
\hline SDSSJ000807.27-103942.7 & 46.9 & 9.29 & 0.29 \\
SDSSJ004241.95+002213.9 & 47.0 & 9.24 & 0.34 \\
SDSSJ021606.41+011509.5 & 47.5 & 9.13 & 1.54 \\
SDSSJ024154.42-004757.5 & 46.9 & 8.88 & 0.77 \\
SDSSJ084036.16+235524.7 & 46.9 & 8.88 & 0.70 \\
SDSSJ101822.96+203558.6 & 47.1 & 9.28 & 0.42 \\
SDSSJ103527.40+445435.6 & 47.1 & 9.63 & 0.21 \\
SDSS105806.16+600826.9 & 47.2 & 9.21 & 0.65 \\
SDSSJ110022.53+484012.6 & 47.0 & 9.22 & 0.37 \\
SDSSJ125659.79-033813.8 & 47.0 & 9.41 & 0.26 \\
SDSSJ131132.92+052751.2 & 47.3 & 9.19 & 0.93 \\
SDSSJ143525.31+400112.2 & 47.4 & 9.14 & 1.31 \\
SDSSJ144412.37+582636.9 & 46.7 & 9.49 & 0.10 \\
SDSSJ151258.36+352533.2 & 47.1 & 8.80 & 1.31 \\
SDSSJ214009.01-064403.9 & 47.2 & 8.79 & 1.61 \\
SDSSJ220119.62-083911.6 & 47.3 & 9.72 & 0.23 \\
SDSSJ222753.07-092951.7 & 46.9 & 9.09 & 0.39 \\
SDSSJ233132.83+010620.9 & 47.7 & 9.84 & 0.50 \\
SDSSJ234657.25+145736.0 & 47.1 & 9.02 & 0.73 \\
\hline
\end{tabular}

Notes. Columns: (1) SDSS identification. (2) Log of Bolometric luminosity in unit of erg s $\mathrm{s}^{-1}$. The associated uncertainty is $10 \%$ the luminosity value. (3) Black hole mass in unit of $M_{\odot}$. (4) Eddington ratio.

\subsubsection{Eddington ratio}

Eddington ratios $\left(L / L_{\text {Edd }}\right)$ have been calculated based on masses obtained from the FWHM AlIII, explained in previous section and following the relation: $L_{\mathrm{Edd}} \approx 1.5 \times 10^{38} \cdot\left(M_{\mathrm{BH}} / M_{\odot}\right)$ $\left[\mathrm{erg} \mathrm{s}^{-1}\right.$ ], as recommended by Netzer \& Marziani (2010). $L / L_{\mathrm{Edd}}$ values for our sample are reported in Table 11. Applying the usual method of propagation of errors and using previous formulae, the typical mean uncertainty in the Eddington ratio is about $30-35 \%$ (only in one case the estimated error is larger than the $50 \%$, in SDSSJ233132.83+010620.9 $L / L_{\text {Edd }} \approx 0.5 \pm 0.3$ ).

The average $L / L_{\mathrm{Edd}}$ value for the GTC-xA sample is $\left\langle L / L_{\text {Edd }}\right\rangle \approx 0.66$, comparable to the one obtained for I $\mathrm{Zw} 1$, a prototype highly accreting quasar at low- $z$. An appropriate sample for a comparison is also the sample \#3 of MS14 that involves $\mathrm{xA}$ quasars in the redshift range $2 \leq z \leq 2.6$ with the AlIII $\lambda 1860$ used as a virial broadening estimator. The obtained average $L / L_{\text {Edd }}$ of the GTC-xA sample is consistent with the value obtained for sample \#3 of $\operatorname{MS} 14\left(L / L_{\text {Edd }} \approx 0.7\right)$, once the estimates are computed with the same bolometric correction (5.4 in this work versus 6.3 in MS14), and the same $L / L_{\text {Edd }}$ constant (MS14 used $1.3 \times 10^{38} \mathrm{erg} \mathrm{s}^{-1}$ ). A Welch-test on the average $L / L_{\text {Edd }}$ confirms that two averages are not significantly different, and therefore the $\mathrm{xA}$ nature of the sources in the present sample.

The scatter in $L / L_{\mathrm{Edd}}$ in the GTC-xA sample after extinction correction is $\sigma_{L / L_{\mathrm{Edd}}} \sim 0.47$ which is somewhat above the one of sample-\#3 of MS14 $\left(\sigma_{\mathrm{L} / \mathrm{L}_{\mathrm{Edd}}} \approx 0.16\right)$, although this may be in part due to the small size of the GTC-xA, with only 19 objects. Also the larger scatter in FWHM may contribute to the $L / L_{\text {Edd }}$ scatter: average FWHM AlIII $\sim 4220 \pm 900 \mathrm{~km} \mathrm{~s}^{-1}$ for MS14 versus an average FWHM AlIII $\sim 4720 \pm 1600 \mathrm{~km} \mathrm{~s}^{-1}$ for the GTC-xA sample. As an example, SDSSJ144412.37+582636.9 has a FWHM AlIII $\sim 8080 \mathrm{~km} \mathrm{~s}^{-1}$ which yields the minimum $L / L_{\text {Edd }}$ value $(0.1)$ in the sample. Additionally this source is a mini-BAL with clear absorptions in its spectrum (see Fig. A.13) introducing additional uncertainties in the extinction correction. In the absence of an orientation correction, a large scatter in the observed FWHM translates into a large scatter in $L / L_{\text {Edd }}$. 


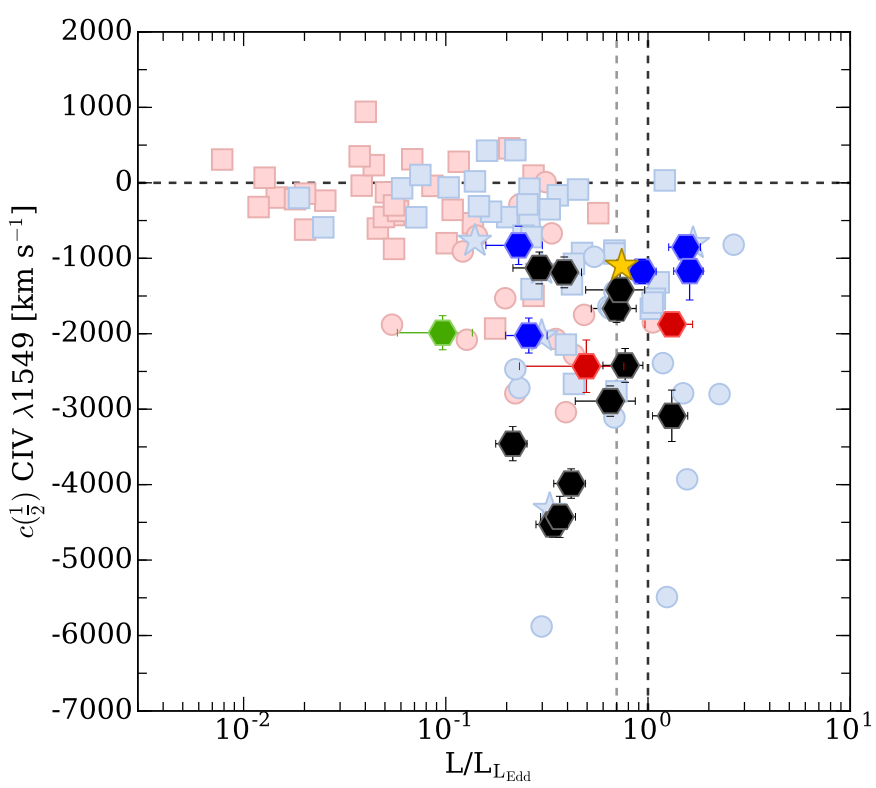

Fig. 9. Behavior of the centroid at $1 / 2$ of total CIV $\lambda 1549$ profile as a function of Eddington ratio. Hexagons represent the GTC-xA sample. The colors of hexagons are the same as Figs. 2 and 3. Blue and red pale symbols correspond to the Population A and B, respectively. Squares and stars correspond to the FOS-A and xA samples (Sulentic et al 2007). Circles are from HE sample Sulentic et al. (2017) sample. Yellow star marks the position of I $\mathrm{Zw} 1$. The black dashed horizontal line indicates $c\left(\frac{1}{2}\right)=0 \mathrm{~km} \mathrm{~s}^{-1}$. Gray and black dashed vertical lines indicate $L / L_{\text {Edd }}=0.2$ and 1.0 , respectively.

A correlation between the $c\left(\frac{1}{2}\right)$ shift and $L / L_{\text {Edd }}$ (Sulentic et al. 2017) implies that highest $c\left(\frac{1}{2}\right)$ values are associated with the highest $L / L_{\text {Edd }}$. Figure 9 shows the plane $c\left(\frac{1}{2}\right)$ vs. $L / L_{\text {Edd }}$, where GTC-xA sources occupy a fairly extreme position, where also I $\mathrm{Zw} 1$ is located. Two third of the 11 sample (unabsorbed sources) have $c\left(\frac{1}{2}\right) \lesssim-2000 \mathrm{~km} \mathrm{~s}^{-1}$, and the blueshifts distribution is more skewed toward high $c\left(\frac{1}{2}\right)$ values than in the sample of S17. Comparing with the HE sample (Sulentic et al. 2017) and FOS-xA and FOS-A sample (Sulentic et al. 2007), we observe that FOS-XA sources (the pale blue squares around $L / L_{\text {Edd }} \sim 1$ ) also cover a similar domain of blueshift amplitudes, while Pop. A sources show lower amplitude shifts, $-900 \mathrm{~km} \mathrm{~s}^{-1}$ on average. This diagram provides further support to the idea that the Eddington ratio is the physical driver of the outflows, and that $\mathrm{xA}$ sources are associated with high Eddington ratio values.

\subsection{Weak-lined and $x A$ quasars}

The Weak-Line Quasars (WLQs) show very low equivalent width in the CIV $\lambda 1549$ and other UV emission lines (Diamond-Stanic et al. 2009; Wu et al. 2012; Shemmer et al. 2010). A standard definition of WLQ involves sources with $W(\mathrm{CIV}) \leq 10 \AA$. WLQs also show strong FeII emission and CIV blueshift. Luo et al. (2015) provides one of the largest samples of WLQ candidates, with detailed measures on shifts and $W$ of CIV for 21 of them. It is an almost exclusively RQ sample as are 4DE1 Population A, and especially xA quasars. Most WLQ candidates are high- $z$ making estimates of source restframe uncertain in many cases. When $\mathrm{H} \beta$ measures are available (Plotkin et al. 2015; Sulentic et al. 2007, 2017), we confirm that WLQs belong to the Population A domain of 4DE1 with $R_{\text {FeII }} \gtrsim 1.0$, independently of $z$ and $L$ (Fig. 9 of Marziani et al.

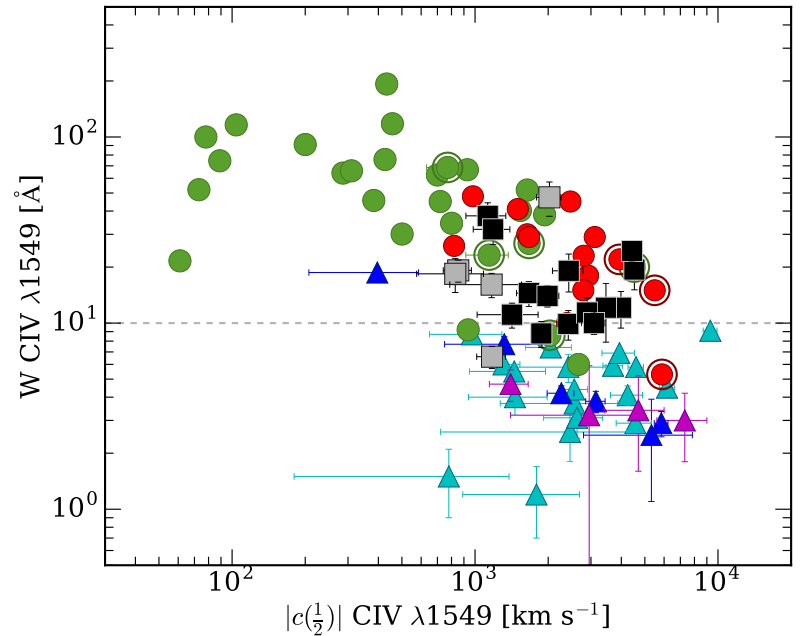

Fig. 10. Relation between rest-frame equivalent width of CIV $\lambda 1549$ and $c\left(\frac{1}{2}\right)$ in $\mathrm{km} \mathrm{s}^{-1}$. Black squares: GTC-xA. Gray squares: BAL GTCxA. Blue triangles: Plotkin et al. (2015), magenta: Wu et al. (2012) and cyan: Luo et al. (2015). Red and green circles correspond to the Pop. A samples from S17 and FOS-A sample, respectively. Circled symbols correspond to FOS-xA and S17-xA.

2016). At least $80 \%$ of WLQs are $x A$ sources and, if doubtful cases (e.g., affected by absorptions, so most likely not real WLQs) are excluded all WLQs might belong to the $\mathrm{xA}$ population. The majority of WLQ show FWHM H $\beta \gtrsim 2000 \mathrm{~km} \mathrm{~s}^{-1}$. WLQ are simply Pop. A sources with strong $R_{\text {FeII }}$, high amplitude CIV blueshift and the lowest values for $W$ CIV.

According to the standard WLQ definition our GTC-xA sample includes three WLQs, excluding BALs. If we consider the prevalence of WLQs among xAs in different samples, we found: four out of 42 in MS14; one out of three (S17); 3 out of 13 in the present sample (excluding BAL and mini-BAL QSOs), and two out of ten (S07), for a total $f_{\mathrm{WLQ}}=n_{\mathrm{WLQ}} / n_{\mathrm{xA}}=10 / 68 \approx 15 \%$.

As we see in Sect. 6.1, the peak of the GTC-xA $W$ CIV $\lambda 1549$ distribution is slightly above $10 \AA$ (see also bottom right panel of Fig. 5). There is no solution of continuity at $W$ CIV $\lambda 1549$ $\approx 10 \AA$. WLQs are located at the low end of the $W$ CIV $\lambda 1549$ distribution of the GTC-xA sources and there is no suggestion that they constitute a special class. Moreover, as in the case $\mathrm{xA}$ sources, WLQs are characterized by extreme outflow velocities. Figure 10 shows the $W$ Civ $\lambda 1549$ vs. CIV $\lambda 1549$ shift domain for the 19 GTC-xA sources of our sample and several WLQs, Pop. xA and Pop. A samples: 19 WLQs from Luo et al. (2015), six WLQs from Plotkin et al. (2015), four WLQ from Wu et al. (2012, which includes PHL 1811 from Wu et al. 2011), three Pop. xA and eleven Pop. A from Sulentic et al. (2017), and five $\mathrm{xA}$ and 23 Pop. A from S07. The measurements in our samples are the centroid at half maximum, while Luo et al. (2015) consider interactive measurement, presumably of the peak shift. In the context of the present analysis, we expect that the effect may not be relevant because, in the case of the largest blueshift, the total profile is shifted so that peak and centroid at half maximum yield consistent values.

The distributions of XA and WLQ blueshifts are largely overlapping, with an excess of WLQs showing blueshifts in the range 5000-10000 $\mathrm{km} \mathrm{s}^{-1}$. A trend between $W$ CIV $\lambda 1549$ and CIV $\lambda 1549$ shift amplitude is suggested, in the sense that the blueshift amplitude appears to increase among weaker CIV $\lambda 1549$ emitters. It is important to consider that this is part of a general trend among quasars, with a much higher statistical significance 
if Pop. A and B at high luminosity are included (Marziani et al. 2016).

Restricting attention to $\mathrm{xA}$ and WLQs means to consider only the extreme end of the equivalent width-shift distribution. Both xAs and WLQs show a coupling between physical and dynamical conditions of the outflowing gas which seems a well-defined, common phenomenon affecting outflowdominated sources (S17). WLQs can therefore interpreted as extreme $\mathrm{xA}$ sources in the context of 4DE1. The previous discussion also validates the assumption that at least the wide majority (if not all) of RQ WLQs are xA quasars. In addition, it suggests that WLQs are an ill-defined class, and that their observational properties should be analyzed within the context of the wider class of xA sources.

At the present time we suggest that observational biases may be playing a serious role. If one considers the data for the most extreme WLQs, extremely low $W$ and extremely high CIV blueshifts, we find that some of the lowest $W$ values in Fig. 10 involve sources with CIV absorptions, that reduce $W \mathrm{CIV}$, and $\mathrm{UV}$ redshift uncertainties, that affect estimates of CIV shifts (Luo et al. 2015). Only a few WLQ candidates have data available for the $\mathrm{H} \beta$ region (Plotkin et al. 2015). Two of these sources report doubtful detections of an extremely broad and very low $W$ CIV (SDSSJ094533.98+100950.1 and SDSSJ141730.92+073320.7). Sources with $c\left(\frac{1}{2}\right)<-5000 \mathrm{~km} \mathrm{~s}^{-1}$ and or $W$ CIV $<3-4 \AA$ require high $\mathrm{S} / \mathrm{N}$ confirmatory spectra. Even if confirmed, we see no evidence for a break in the distribution at $W$ CIV $=10 \AA$ suggesting that WLQ are the most extreme Pop. A quasars (xA), with a lowest values for $W$ CIV.

\subsection{Outflows and feedback}

Hereafter we will consider only median luminosity estimates after extinction correction excluding BAL and mini-BAL sources. The median value of the blueshifted component (excluding the BAL cases) is $\log L_{\mathrm{BLUE}} \approx 44.0 \pm 0.3\left[\mathrm{erg} \mathrm{s}^{-1}\right]$. It is relatively straightforward to compute the mass of ionized gas needed to sustain the Civ $\lambda 1549$ blue shifted component luminosity: $M^{\text {ion }} \sim 95 Z_{10}^{-1} n_{9}^{-1} M_{\odot}$, where the density is assumed $10^{9} \mathrm{~cm}^{-3}$, and the relation is normalized to a metal content ten times solar (e.g., Cano-Díaz et al. 2012; Marziani et al. 2016, 2017a). Computation of the mass outflow rate, of its thrust, and of its kinetic power requires several assumptions (discussed in the recent review of Marziani et al. 2017a). In the present context, we assume a BLR radius estimated from the radius luminosity relation derived by Kaspi et al. (2007). At the average continuum luminosity after extinction correction $\lambda L_{\lambda}(1350) \approx 2.4 \times 10^{46} \mathrm{erg} \mathrm{s}^{-1}$, the $r_{\mathrm{BLR}} \approx 2.6 \times$ $10^{17} \mathrm{~cm} \approx 0.086 \mathrm{pc}$. The mass outflow rate can then be written as: $\dot{M}^{\text {ion }} \sim 150 L_{45} v_{\mathrm{o}, 5000} r_{\mathrm{BLR}, 0.1 \mathrm{pc}}^{-1} Z_{10}^{-1} n_{9}^{-1} M_{\odot}\left[\mathrm{yr}^{-1}\right]$, where the outflow radial velocity is in units of $5000 \mathrm{~km} \mathrm{~s}^{-1}$. For the median centroid displacement of the blue shifted component $\approx-3560 \mathrm{~km} \mathrm{~s}^{-1}$, we obtain a $\dot{M}^{\text {ion }} \approx 12.5 M_{\odot} \mathrm{yr}^{-1}$. The final thrust can be expressed as: $\dot{M}^{\text {ion }} k v_{\mathrm{o}} \sim 5 \times$ $10^{36} L_{45} k v_{\mathrm{o}, 5000}^{2} r_{\mathrm{BLR}, 0.1 \mathrm{pc}}^{-1} Z_{10}^{-1} n_{9}^{-1}\left[\mathrm{~g} \mathrm{~cm} \mathrm{~s}^{-2}\right.$ ], where we have now introduced a factor $k$ that takes into account that the gas within the BLR is still being accelerated by radiation forces. In the scenario of Netzer \& Marziani (2010), for Eddington ratio $\rightarrow 1$, as it is likely the case of $\mathrm{xA}$ sources, $k$ can be as high as ten. We prudentially assume $k=5$, and that the full blue shifted component is accelerated to $k v$. For the average values of the $\mathrm{xA}$ sources we obtain $\dot{M}^{\text {ion }} k v_{\mathrm{o}} \sim 1.5 \times 10^{36} \mathrm{~g} \mathrm{~cm} \mathrm{~s}^{-2}$. The kinetic power of the outflow is $\dot{\epsilon} \sim 1.2 \times 10^{45} L_{45} k^{2} v_{0,5000}^{3} r_{0.1 \mathrm{pc}}^{-1} Z_{10}^{-1} n_{9}^{-1} \mathrm{erg} \mathrm{s}^{-1}$, and becomes $\dot{\epsilon} \sim 1.3 \times 10^{45} \mathrm{erg} \mathrm{s}^{-1}$ for the average parameter values of the xA sample.

The large outflow radial velocity (the average $c\left(\frac{1}{2}\right)$ of the blue shifted component is close to $\sim-4000 \mathrm{~km} \mathrm{~s}^{-1}$ ), as well as the high fraction of the blue shifted component to the total CIV $\lambda 1549$ luminosity ( $\sim .45$, Sect. 5.4$)$ ensure the maximization of the quasar mechanical feedback even if $W$ Civ $\lambda 1549$ is in general relatively low (cf., King \& Muldrew 2016). The ratio $\dot{\epsilon} / L \sim 0.01$ comes close to the threshold value for exerting significant mechanical feedback on the entire host galaxies especially if the quasar is radiating at high Eddington ratio and at high luminosity (e.g., King \& Pounds 2015, and references therein). High luminosity outflows may be even more powerful if one considers that the CIV $\lambda 1549$ emission traces only a phase of multiphase outflowing medium (Tombesi et al. 2010; Harrison et al. 2014; Cicone et al. 2014; Carniani et al. 2015; Feruglio et al. 2015). On the other hand, if $Z$ is as high as $100 Z_{\odot}$, then all estimates should be reduced by a factor of ten. This emphasizes the need of accurate metallicity estimates not only to study the enrichment of the ISM by nuclear outflows, but also to ascertain their dynamical and evolutionary relevance on the host galaxy.

\section{Conclusion}

We present a sample of 19 quasar satisfying the 4DE1 selection criteria for finding high redshift highly accretors xA sources. We provide a description of the rest-frame UV spectra of our candidate sources. Our GTC-xA sample shows the same characteristics of $\mathrm{xA}$ quasars at low $-z$, of which a prototype is $\mathrm{I} \mathrm{Zw} 1$. Figure 2 shows that GTC-xA sample occupies a very restricted AlIII $\lambda 1860 /$ SiIII] $\lambda 1892$ vs. CIII] $\lambda 1909 /$ SiIII] $\lambda 1892$ domain space, compared to more general quasar populations. GTC-xA sources show different spectroscopic properties than other Pop. A (Bins A1, A2) quasars. We show that our sample is likely radiating close to the Eddington limit $\left(L / L_{\text {Edd }} \geq 0.2\right)$ with an average consistent with the one computed on the MS14 xA sample.

AlIII $\lambda 1860$ and SiIII] $\lambda 1892$ lines are well-modeled with a symmetric profile that is narrower than that of CIV $\lambda 1549$ total profile. These results support our previous suggestions that AlIII $\lambda 1860$ is likely a reliable virial estimator in analog of $\mathrm{H} \beta$ for lower $z$ quasars. On the converse, the FWHM CIV remains unreliable as a virial estimator showing no significant correlation with FWHM AlIII $\lambda 1860$. On the other hand, CIV $\lambda 1549$ profiles in GTC-xA sources show large blueshifts, $\left|c\left(\frac{1}{2}\right)\right|=$ $1000-5000 \mathrm{~km} \mathrm{~s}^{-1}$. Figure 3 shows evidence for a correlation between FWHM and $c\left(\frac{1}{2}\right)$ for CIV $\lambda 1549$. The uniformity of the source distribution along the full blueshift range can be used to argue for a quasi isotropic wind in XA quasars. Another possibility sees a slight source excess around $c\left(\frac{1}{2}\right) \sim-2000 \mathrm{~km} \mathrm{~s}^{-1}$. Only a larger sample can permit a more robust speculation.

The emission line spectrum of the GTC-xA sources is characterized by low equivalent width of the most prominent emission lines. WLQs appear as extreme xA sources and not as an independent class. All WLQ might be xA sources, if we consider observations with the $\mathrm{H} \beta$ spectral range covered. The appearance of the XA spectrum at high- $L$ is of very low ionization. FeII and FeIII contributions appear to be more prominent in the spectra, although this is in part due to the low equivalent width of all the emission features. The FeIII strength is only in apparent contradiction with very low values of the ionization parameter: $\mathrm{Fe}^{+2}$ 
becomes the dominant ionization stage of iron at the expense of $\mathrm{Fe}^{+3}$, therefore increasing $\mathrm{Fe}^{+2}$ with respect to $\mathrm{Fe}^{+1}$.

Numerous results merit discussion and further study. Among them, we list two that are relevant to our global understanding of the black hole-host galaxy connection. Firstly, the CIV shift and the luminosity suggest high values of mass outflow rate, thrust, and kinetic power of the outflow. This is expected for sources of high- $L$ and maximized radiation forces per unit mass. However, more refined computations are needed to assess the relevance of the outflow dynamical parameters for feedback effects on the host galaxy. And secondly, the intensity ratio HeII $\lambda 1640 / \operatorname{CIV} \lambda 1549$ requires high $Z\left(\gtrsim 50 Z_{\odot}\right)$ to be consistent with a low-ionization, high density $\left(\log U \sim-3, \log n_{\mathrm{H}} \sim\right.$ $13 \mathrm{~cm}^{-3}$ ) that we found from our data and that is consistent with the analysis of (N12). This result claims for accurate estimates of the line emitting region chemical composition that are still missing today.

This preliminary paper provides a description of several key properties of $\mathrm{xA}$ at high- $L$. One of the most interesting result is that AlIII $\lambda 1860$ is providing a tracer of the dynamics of a virialized LIL-emitting region. If this is true at least approximately AlIII $\lambda 1860$ may offers a virial broadening estimator. The AlIII $\lambda 1860$ is a resonant doublet which implies resonant absorption and therefore efficient acceleration by continuum scattering. Then a non-virial contribution due to outflows may remain unresolved in the AlIII $\lambda 1860$ profile. Simultaneous observations of the AlIII $\lambda 1860$ and of the $\mathrm{H} \beta$ spectral ranges are needed to make an exhaustive assessment of AlIII $\lambda 1860$ as a virial broadening estimator. The conceptual validity of luminosity estimates based on the $\mathrm{H} \beta$ line width measurements has been confirmed by the Hubble diagrams shown by MS14 and Negrete et al. (2017). The large scatter displayed by quasars with respect to supernovæ demands that random and systematic sources of error should be understood and, if possible, corrected or reduced before the xA sources can be exploited as useful distance indicators for cosmology.

A reliable virial broadening estimator would make possible an application of the virial luminosity estimates proposed by MS14 to a large number of quasars in the redshift range $1 \lesssim z \lesssim 3.5$. This redshift range is especially well suited for obtaining constraints on the cosmic density of matter $\Omega_{\mathrm{M}}$, since the effect of the cosmological constant in the dynamics of the Universe is felt only at $z \lesssim 1$.

Acknowledgements. MLMA acknowledges a CONACyT postdoctoral fellowship. AdO, MLMA and JWS acknowledge financial support from the Spanish Ministry for Economy and Competitiveness through grants AYA2013-42227-P and AYA2016-76682-C3-1-P. MLMA, PM and MDO acknowledge funding from the INAF PRIN-SKA 2017 program 1.05.01.88.04. JP acknowledge financial support from the Spanish Ministry for Economy and Competitiveness through grants AYA2013-40609-P and AYA2016-76682-C3-3-P. MLMA is thankful for the kind hospitality at the Padova Astronomical Observatory, PM for the hospitality at IAA-CSIC. DD and AN acknowledge support from CONACyT through grant CB221398. DD and AN thank also for support from grant IN108716 PAPIIT, UNAM. We would like to thank Drs. J. Masegosa, I. Marquez, M. Povic and S. Cazzoli for all the fruitful discussions on the GANG meetings. This work is based on observations made with the Gran Telescopio Canarias (GTC), installed in the Spanish Observatorio del Roque de los Muchachos of the Instituto de Astrofísica de Canarias, in the island of La Palma. We thank all the GTC Staff for their support with the observations. This research has made use of the NASA/IPAC Extragalactic Database (NED) which is operated by the Jet Propulsion Laboratory, California Institute of Technology, under contract with the National Aeronautics and Space Administration.

\section{References}

Andrae, R. 2010, ArXiv e-prints [arXiv:1009.2755]

Bachev, R., Marziani, P., Sulentic, J. W., et al. 2004, ApJ, 617, 171
Baldwin, J. A., Ferland, G. J., Korista, K. T., et al. 1996, ApJ, 461, 664 Baldwin, J. A., Ferland, G. J., Korista, K. T., Hamann, F., \& LaCluyzé, A. 2004, ApJ, 615, 610

Barlow, R. 1989, in The Manchester Physics Series (New York: Wiley)

Bellazzini, M. 2007, Light Losses Due to variation of seeing with wavelength, Internal Protocol on Data Reduction According to Gaia DPAC, GAIA-C5TN-UB-JMC-004

Bensch, K., del Olmo, A., Sulentic, J., Perea, J., \& Marziani, P. 2015, JA\&A, 36, 467

Bischetti, M., Piconcelli, E., Vietri, G., et al. 2017, A\&A, 598, A122

Bisogni, S., di Serego Alighieri, S., Goldoni, P., et al. 2017, A\&A, 603, A1

Boroson, T. A. 2002, ApJ, 565, 78

Boroson, T. A., \& Green, R. F. 1992, ApJS, 80, 109

Boyle, B. J., Shanks, T., Croom, S. M., et al. 2000, MNRAS, 317, 1014

Brotherton, M. S., Tran, H. D., Becker, R. H., et al. 2001, ApJ, 546, 775

Brotherton, M. S., Runnoe, J. C., Shang, Z., \& DiPompeo, M. A. 2015, MNRAS, 451, 1290

Brühweiler, F., \& Verner, E. 2008, ApJ, 675, 83

Cano-Díaz, M., Maiolino, R., Marconi, A., et al. 2012, A\&A, 537, L8

Carniani, S., Marconi, A., Maiolino, R., et al. 2015, A\&A, 580, A102

Carswell, R. F., Mountain, C. M., Robertson, D. J., et al. 1991, ApJ, 381, L5

Cegłowski, M., Kunert-Bajraszewska, M., \& Roskowiński, C. 2015, MNRAS, 450, 1123

Cicone, C., Maiolino, R., Sturm, E., et al. 2014, A\&A, 562, A21

Clowes, R. G., Haberzettl, L., Raghunathan, S., et al. 2016, MNRAS, 460, 1428

Coatman, L., Hewett, P. C., Banerji, M., \& Richards, G. T. 2016, MNRAS, 461, 647

Coatman, L., Hewett, P. C., Banerji, M., et al. 2017, MNRAS, 465, 2120

Cracco, V., Ciroi, S., Berton, M., et al. 2016, MNRAS, 462, 1256

Czerny, B., Beaton, R., Bejger, M., et al. 2018, Space Sci. Rev., 214, 32

Denney, K. D., Horne, K., Brandt, W. N., et al. 2016, ApJ, 833, 33

Diamond-Stanic, A. M., Fan, X., Brandt, W. N., et al. 2009, ApJ, 699, 782

D’Onofrio, M., \& Burigana, C. 2009, Questions of Modern Cosmology: Galileo's Legacy (Springer Science \& Business Media)

Du, P., Lu, K.-X., Hu, C., et al. 2016a, ApJ, 820, 27

Du, P., Wang, J.-M., Hu, C., et al. 2016b, ApJ, 818, L14

Elvis, M., Wilkes, B. J., McDowell, J. C., et al. 1994, ApJS, 95, 1

Eracleous, M., \& Halpern, J. P. 2003, ApJ, 599, 886

Espey, B. R., Carswell, R. F., Bailey, J. A., Smith, M. G., \& Ward, M. J. 1989, ApJ, 342, 666

Ferland, G. J., Porter, R. L., van Hoof, P. A. M., et al. 2013, Rev. Mex. Astron. Astrofis., 49, 137

Feruglio, C., Fiore, F., Carniani, S., et al. 2015, A\&A, 583, A99

Fraix-Burnet, D., Marziani, P., D’Onofrio, M., \& Dultzin, D. 2017, Front. Astron. Space Sci., 4, 1

Gallerani, S., Maiolino, R., Juarez, Y., et al. 2010, A\&A, 523, A85

Gaskell, C. M. 1982, ApJ, 263, 79

Gaskell, C. M., Brandt, W. N., Dietrich, M., Dultzin-Hacyan, D., \& Eracleous, M. 1999, ASP Conf. Ser., 175

Giacconi, R., Branduardi, G., Briel, U., et al. 1979, ApJ, 230, 540

Gordon, K. D., \& Clayton, G. C. 1998, ApJ, 500, 816

Graham, M. J., Clowes, R. G., \& Campusano, L. E. 1996, MNRAS, 279, 1349

Hall, P. B., Anderson, S. F., Strauss, M. A., et al. 2002, ApJS, 141, 267

Hamann, F., \& Ferland, G. 1993, ApJ, 418, 11

Hamann, F., \& Ferland, G. 1999, ARA\&A, 37, 487

Harris, D. W., Jensen, T. W., Suzuki, N., et al. 2016, AJ, 151, 155

Harrison, C. M., Alexander, D. M., Mullaney, J. R., \& Swinbank, A. M. 2014, MNRAS, 441, 3306

Hu, C., Wang, J.-M., Ho, L. C., et al. 2008, ApJ, 683, L115

Johansson, S., \& Hansen, J. E. 1988, in IAU Colloq. 94: Physics of Formation of FE II Lines Outside LTE, eds. R. Viotti, A. Vittone, \& M. Friedjung, Astrophys. Space Sci. Lib., 138, 235

Johansson, S., Brage, T., Leckrone, D. S., Nave, G., \& Wahlgren, G. M. 1995, ApJ, 446, 361

Kaspi, S., Brandt, W. N., Maoz, D., et al. 2007, ApJ, 659, 997

Kellermann, K. I., Sramek, R., Schmidt, M., Shaffer, D. B., \& Green, R. 1989, AJ, 98, 1195

King, A., \& Muldrew, S. I. 2016, MNRAS, 455, 1211

King, A., \& Pounds, K. 2015, ARA\&A, 53, 115

Kovačević, J., Popović, L. Č., \& Dimitrijević, M. S. 2010, ApJS, 189, 15

Kriss, G. 1994, ASP Conf. Ser., 61, 437

Kuraszkiewicz, J. K., Green, P. J., Crenshaw, D. M., et al. 2004, ApJS, 150, 165 Kurucz, R., \& Bell, B. 1995, Atomic Line Data, Kurucz CD-ROM No. 23 (Cambridge, MA: Smithsonian Astrophysical Observatory), 23

La Franca, F., Bianchi, S., Ponti, G., Branchini, E., \& Matt, G. 2014, ApJ, 787, L12

Laor, A., Jannuzi, B. T., Green, R. F., \& Boroson, T. A. 1997, ApJ, 489, 656

Luo, B., Brandt, W. N., Hall, P. B., et al. 2015, ApJ, 805, 122 
Malkan, M. A., \& Sargent, W. L. W. 1982, ApJ, 254, 22

Marinello, A. O. M., Rodriguez-Ardila, A., Garcia-Rissmann, A., Sigut, T. A. A., \& Pradhan, A. K. 2016, ApJ, 820, 116

Martínez-Aldama, M. L., Dultzin, D., Marziani, P., et al. 2015, ApJS, 217, 3

Martínez-Aldama, M. L., Del Olmo, A., Marziani, P., et al. 2017, Front. Astron. Space Sci., 4, 29

Marziani, P., \& Sulentic, J. W. 2014, MNRAS, 442, 1211

Marziani, P., Sulentic, J. W., Dultzin-Hacyan, D., Calvani, M., \& Moles, M 1996, ApJS, 104, 37

Marziani, P., Sulentic, J. W., Zwitter, T., Dultzin-Hacyan, D., \& Calvani, M 2001, ApJ, 558, 553

Marziani, P., Sulentic, J. W., Zamanov, R., et al. 2003a, ApJS, 145, 199

Marziani, P., Zamanov, R. K., Sulentic, J. W., \& Calvani, M. 2003b, MNRAS, 345,1133

Marziani, P., Sulentic, J. W., Stirpe, G. M., Zamfir, S., \& Calvani, M. 2009, A\&A, 495, 83

Marziani, P., Sulentic, J. W., Negrete, C. A., et al. 2010, MNRAS, 409, 1033

Marziani, P., Sulentic, J. W., Plauchu-Frayn, I., \& del Olmo, A. 2013, ApJ, 764, 150

Marziani, P., Martínez Carballo, M. A., Sulentic, J. W., et al. 2016, Ap\&SS, 361

Marziani, P., Negrete, C. A., Dultzin, D., et al. 2017a, Front. Astron. Space Sci., 4,16

Marziani, P., Olmo, A., Martínez-Aldama, M., et al. 2017b, Atoms, 5, 33

Marziani, P., Dultzin, D., Sulentic, J. W., et al. 2018, Front. Astron. Space Sci. 5,6

Mathews, W. G., \& Ferland, G. J. 1987, ApJ, 323, 456

Matsuoka, K., Silverman, J. D., Schramm, M., et al. 2013, ApJ, 771, 64

Mejía-Restrepo, J. E., Trakhtenbrot, B., Lira, P., Netzer, H., \& Capellupo, D. M. 2016, MNRAS, 460

Mejía-Restrepo, J. E., Trakhtenbrot, B., Lira, P., \& Netzer, H. 2018, MNRAS, 478, 1929

Mineshige, S., Kawaguchi, T., Takeuchi, M., \& Hayashida, K. 2000, PASJ, 52, 499

Negrete, A., Dultzin, D., Marziani, P., \& Sulentic, J. 2012, ApJ, 757, 62

Negrete, C. A., Dultzin, D., Marziani, P., \& Sulentic, J. W. 2013, ApJ, 771, 31

Negrete, C. A., Dultzin, D., Marziani, P., \& Sulentic, J. W. 2014, ApJ, 794, 95

Negrete, C. A., Dultzin, D., Marziani, P., et al. 2017, Front. Astron. Space Sci. 4,59

Negrete, C. A., Dultzin, D., Marziani, P., et al. 2018, A\&A, in press, DOI 10.1051/0004-6361/201833285

Netzer, H., \& Marziani, P. 2010, ApJ, 724, 318

Netzer, H., Lira, P., Trakhtenbrot, B., Shemmer, O., \& Cury, I. 2007, ApJ, 671, 1256

Park, D., Woo, J.-H., Denney, K. D., \& Shin, J. 2013, ApJ, 770, 87

Penston, M. V. 1987, MNRAS, 229, 1P

Plotkin, R. M., Shemmer, O., Trakhtenbrot, B., et al. 2015, ApJ, 805, 123

Richards, G. T., Lacy, M., Storrie-Lombardi, L. J., et al. 2006, ApJS, 166, 470

Richards, G. T., Kruczek, N. E., Gallagher, S. C., et al. 2011, AJ, 141, 167

Scaringi, S., Cottis, C. E., Knigge, C., \& Goad, M. R. 2009, MNRAS, 399, 2231
Schneider, D. P., Hall, P. B., Richards, G. T., et al. 2005, AJ, 130, 367

Shemmer, O., Netzer, H., Maiolino, R., et al. 2004, ApJ, 614, 547

Shemmer, O., Trakhtenbrot, B., Anderson, S. F., et al. 2010, ApJ, 722, L152 Shen, Y., \& Liu, X. 2012, ApJ, 753, 125

Shen, Y., Brandt, W. N., Richards, G. T., et al. 2016, ApJ, 831, 7

Shin, J., Woo, J.-H., Nagao, T., \& Kim, S. C. 2013, ApJ, 763, 58

Sigut, T. A. A., \& Pradhan, A. K. 1998, ApJ, 499, L139

Sigut, T. A. A., Pradhan, A. K., \& Nahar, S. N. 2004, ApJ, 611, 81

Sniegowska, M., Czerny, B., You, B., et al. 2018, A\&A, 613, A38

Sulentic, J., \& Marziani, P. 2015, Front. Astron. Space Sci., 2, A6

Sulentic, J. W., Marziani, P., \& Dultzin-Hacyan, D. 2000a, ARA\&A, 38, 521

Sulentic, J. W., Zwitter, T., Marziani, P., \& Dultzin-Hacyan, D. 2000b, ApJ, 536, L5

Sulentic, J. W., Marziani, P., Zamanov, R., et al. 2002, ApJ, 566, L71

Sulentic, J. W., Zamfir, S., Marziani, P., et al. 2003, ApJ, 597, L17

Sulentic, J. W., Dultzin-Hacyan, D., Marziani, P., et al. 2006a, Rev. Mex. Astron. Astrofis., 42, 23

Sulentic, J. W., Repetto, P., Stirpe, G. M., et al. 2006b, A\&A, 456, 929

Sulentic, J. W., Bachev, R., Marziani, P., Negrete, C. A., \& Dultzin, D. 2007, ApJ, 666, 757

Sulentic, J. W., Zamfir, S., Marziani, P., \& Dultzin, D. 2008, Rev. Mex. Astron. Astrofis. Conf. Ser., 32, 51

Sulentic, J. W., Marziani, P., del Olmo, A., et al. 2014a, A\&A, 570, A96

Sulentic, J. W., Marziani, P., Dultzin, D., D’Onofrio, M., \& del Olmo, A. 2014b, J. Phys. Conf. Ser., 565, 012018

Sulentic, J. W., Martínez-Carballo, M. A., Marziani, P., et al. 2015, MNRAS, 450, 1916

Sulentic, J. W., del Olmo, A., Marziani, P., et al. 2017, A\&A, 608, A122

Sun, J., \& Shen, Y. 2015, ApJ, 804, L15

Tombesi, F., Sambruna, R. M., Reeves, J. N., et al. 2010, ApJ, 719, 700

Trakhtenbrot, B., \& Netzer, H. 2012, MNRAS, 427, 3081

Trump, J. R., Hall, P. B., Reichard, T. A., et al. 2006, ApJS, 165,

Tully, R. B., \& Fisher, J. R. 1977, A\&A, 54, 661

Vanden Berk, D. E., Richards, G. T., Bauer, A., et al. 2001, AJ, 122, 549

Verner, E., Bruhweiler, F., Verner, D., et al. 2004, ApJ, 611, 780

Véron-Cetty, M.-P., \& Véron, P. 2010, A\&A, 518, A10

Vestergaard, M., \& Peterson, B. M. 2006, ApJ, 641, 689

Vestergaard, M., \& Wilkes, B. J. 2001, ApJS, 134, 1

Vietri, G., Piconcelli, E., Bischetti, M., et al. 2018, A\&A, 617, A81

Wang, J.-M., Du, P., Valls-Gabaud, D., Hu, C., \& Netzer, H. 2013, Phys. Rev. Lett., 110, 081301

Wang, J.-M., Du, P., Hu, C., et al. 2014, ApJ, 793, 108

Weymann, R. J., Morris, S. L., Foltz, C. B., \& Hewett, P. C. 1991, ApJ, 373, 23

Wills, D., \& Netzer, H. 1979, ApJ, 233, 1

Wu, J., Brandt, W. N., Hall, P. B., et al. 2011, ApJ, 736, 28

Wu, J., Brandt, W. N., Anderson, S. F., et al. 2012, ApJ, 747, 10

York, D. G., Khare, P., Vanden Berk, D., et al. 2006, MNRAS, 367, 945

Zamfir, S., Sulentic, J. W., \& Marziani, P. 2008, MNRAS, 387, 856

Zamfir, S., Sulentic, J. W., Marziani, P., \& Dultzin, D. 2010, MNRAS, 403, 1759 


\section{Appendix A: Rest-frame spectra and multicomponent fits}

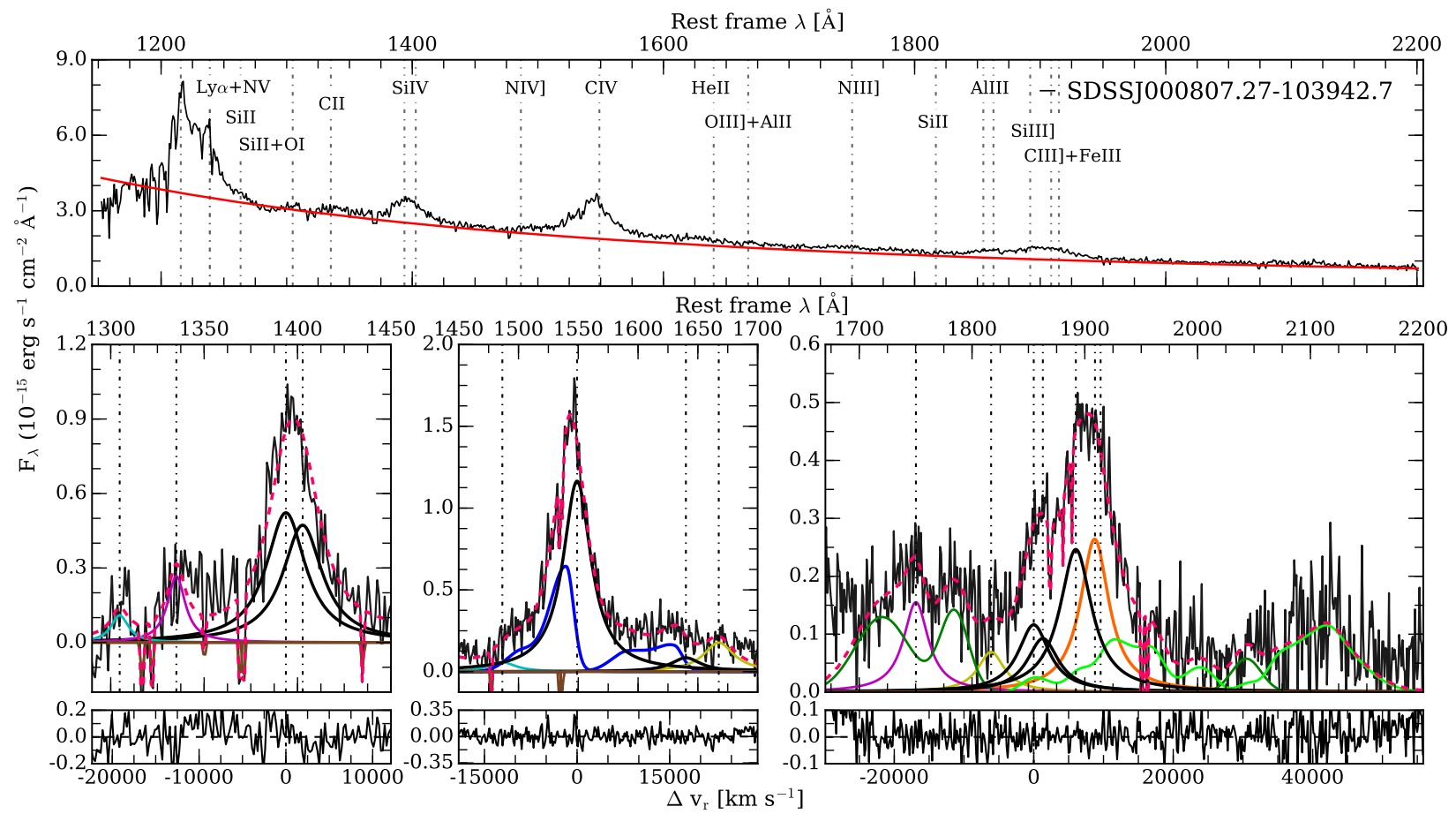

Fig. A.1. Top panel: calibrated rest-frame spectrum of SDSS000807.27-103942.7 before continuum substraction. Abscissa is rest-frame wavelength in $\AA$, while ordinate is specific flux in units $10^{-15} \mathrm{erg} \mathrm{s}^{-1} \mathrm{~cm}^{-2} \mathrm{~Hz}^{-1}$. Global or local continuum are specified by a continuous line. Dot-dashed vertical lines identify the position at rest-frame of the strongest emission lines. Bottom panel: multicomponent fits after continuum subtraction for the SiIV $\lambda 1397$, CIV $\lambda 1549$ and $1900 \AA$ Alend spectral ranges. In all the panels the continuous black line marks the broad component at rest-frame associated to SiIV $\lambda 1397$, CIV $\lambda 1549$, AlIII $\lambda 1860$ and SiIII] $\lambda 1892$ respectively, while the blue one corresponds to the blueshifted component associated to each emission. Dashed pink line marks the fit to the whole spectrum. Absorption lines are indicated by a brown line. Dot-dashed vertical lines correspond to the rest-frame of each emission line. In the SiIV $\lambda 1397$ spectral range, the cyan line marks the contribution of OI + S II 1304 blend, while the magenta line corresponds to the CII $\lambda 1335$ emission line. In the CIV $\lambda 1549$ region, NIV] $\lambda 1486$ is represented by a cyan line, while the yellow one corresponds to the OIII] $\lambda 1663+$ AlII $\lambda 1670$ blend. In the $1900 \AA$ blend range, FeIII and FeII contributions are traced by dark and pale green lines respectively, magenta lines marks the NIII] $\lambda 1750$ and the yellow one corresponds to the SiII $\lambda 1816$. Lower panels correspond to the residuals, abscissa is in radial velocity units $\mathrm{km} \mathrm{s}^{-1}$.

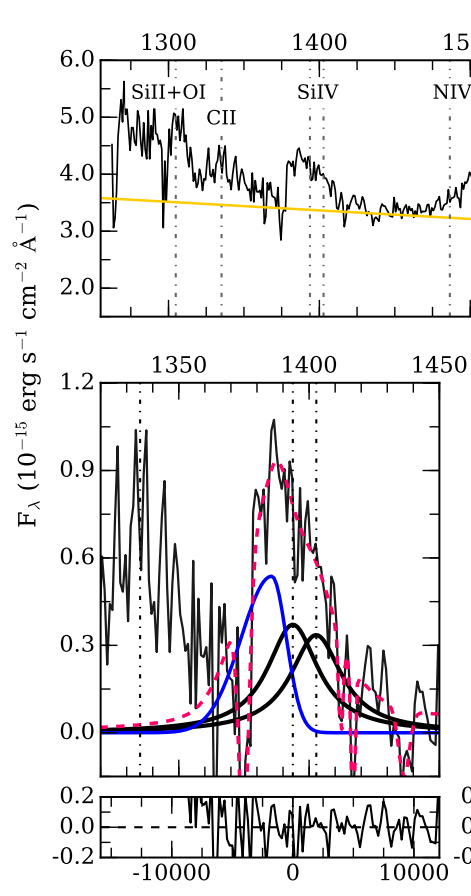

Rest frame $\lambda[\AA]$

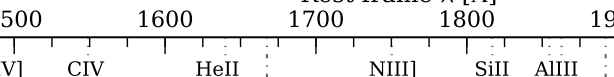

1900

2000

2100

2200

$\begin{array}{ccccc}\vdots & \text { OIII]+AlII } & \vdots & \vdots & \vdots \\ \vdots & & \text { SiII] }\end{array}$

- SDSSJ004241.95+002213.9

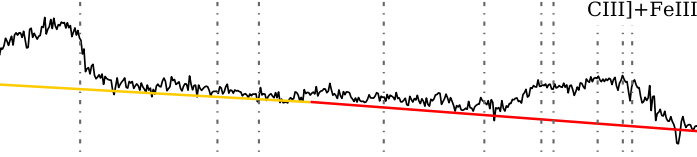



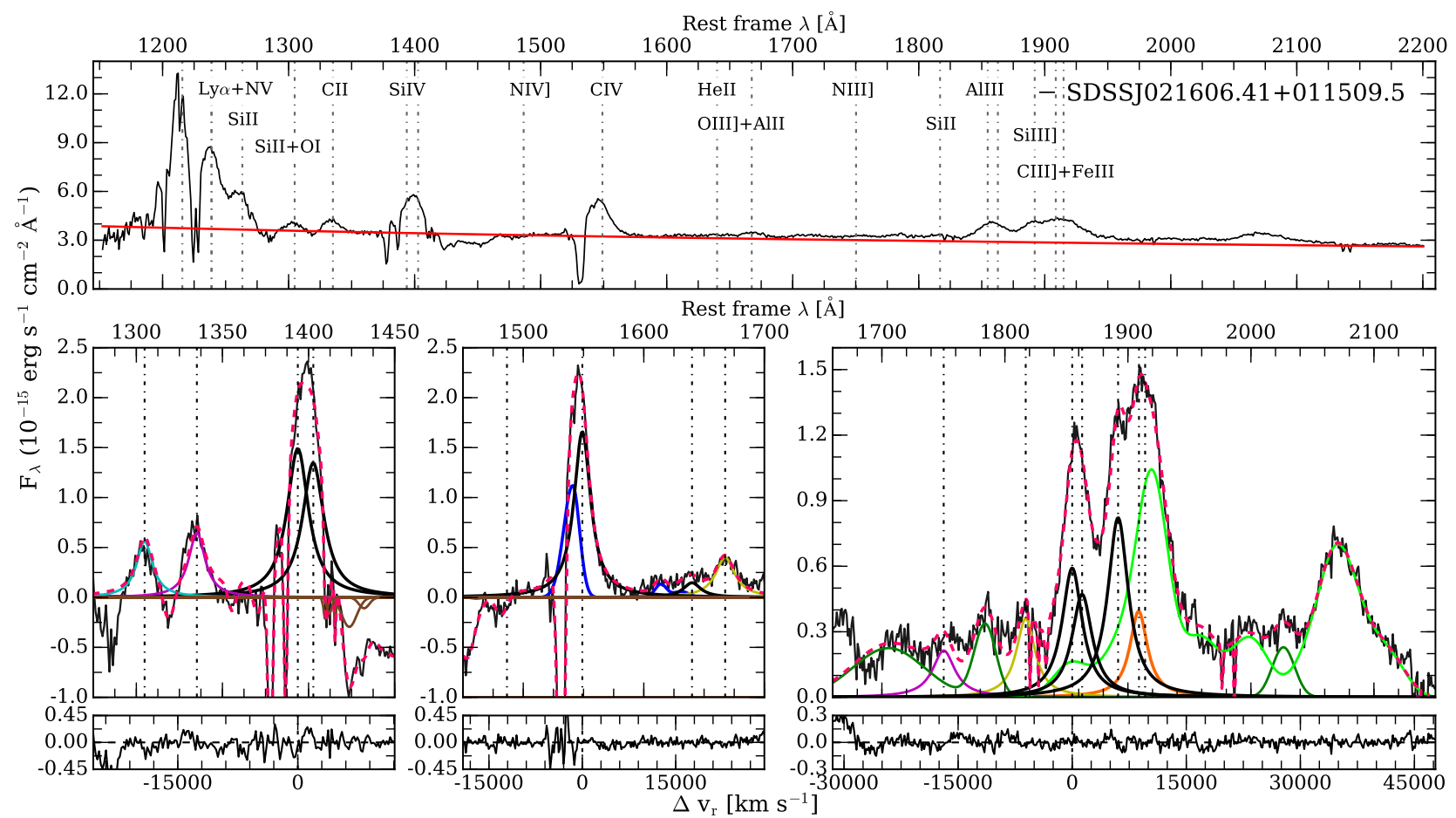

Fig. A.3. Same as Fig. A.1, for SDSSJ021606.41+011509.5.

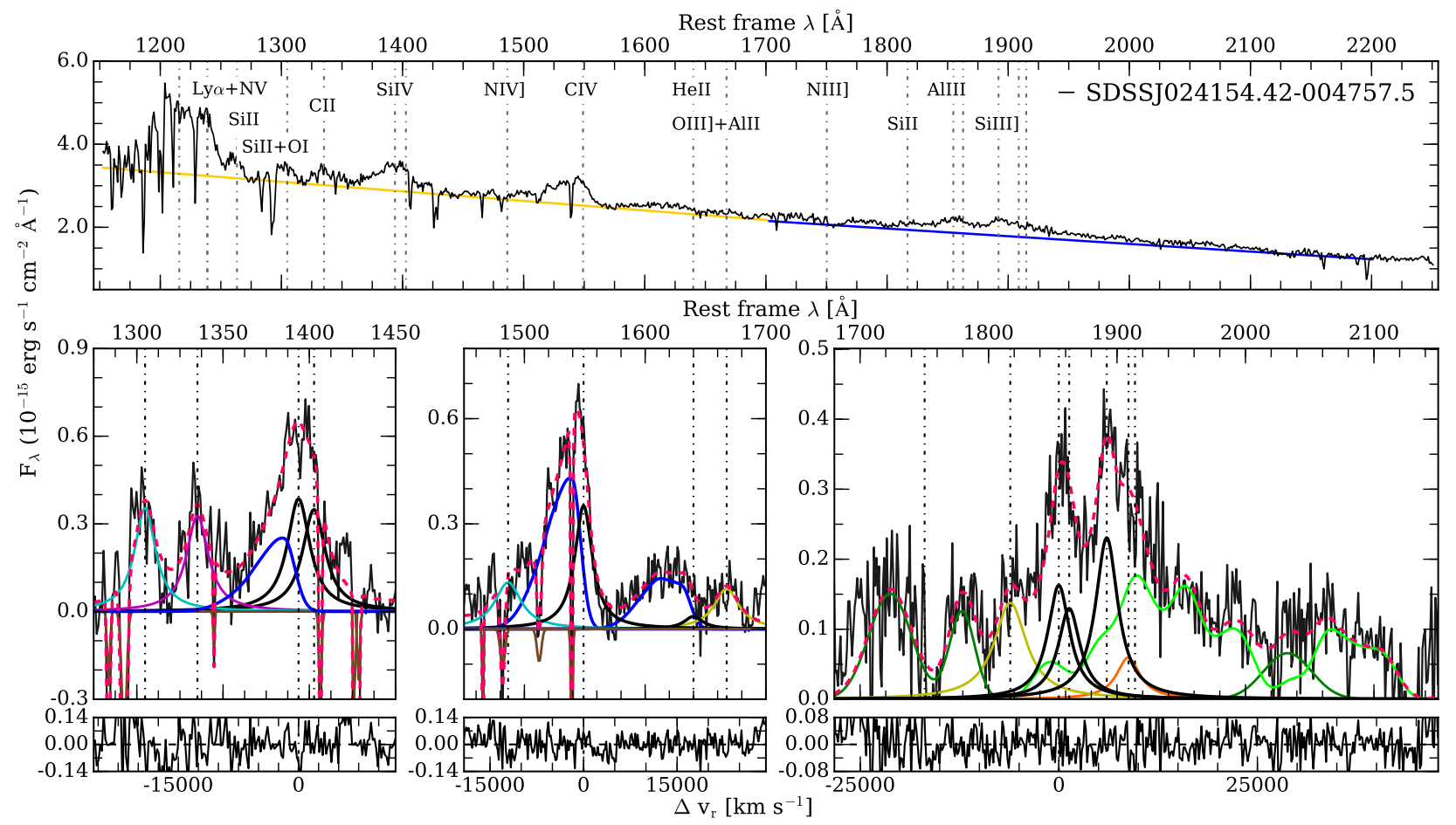

Fig. A.4. Same as Fig. A.1, for SDSSJ024154.42-004757.5. 
M. L. Martínez-Aldama et al.: Extreme quasars at high redshift

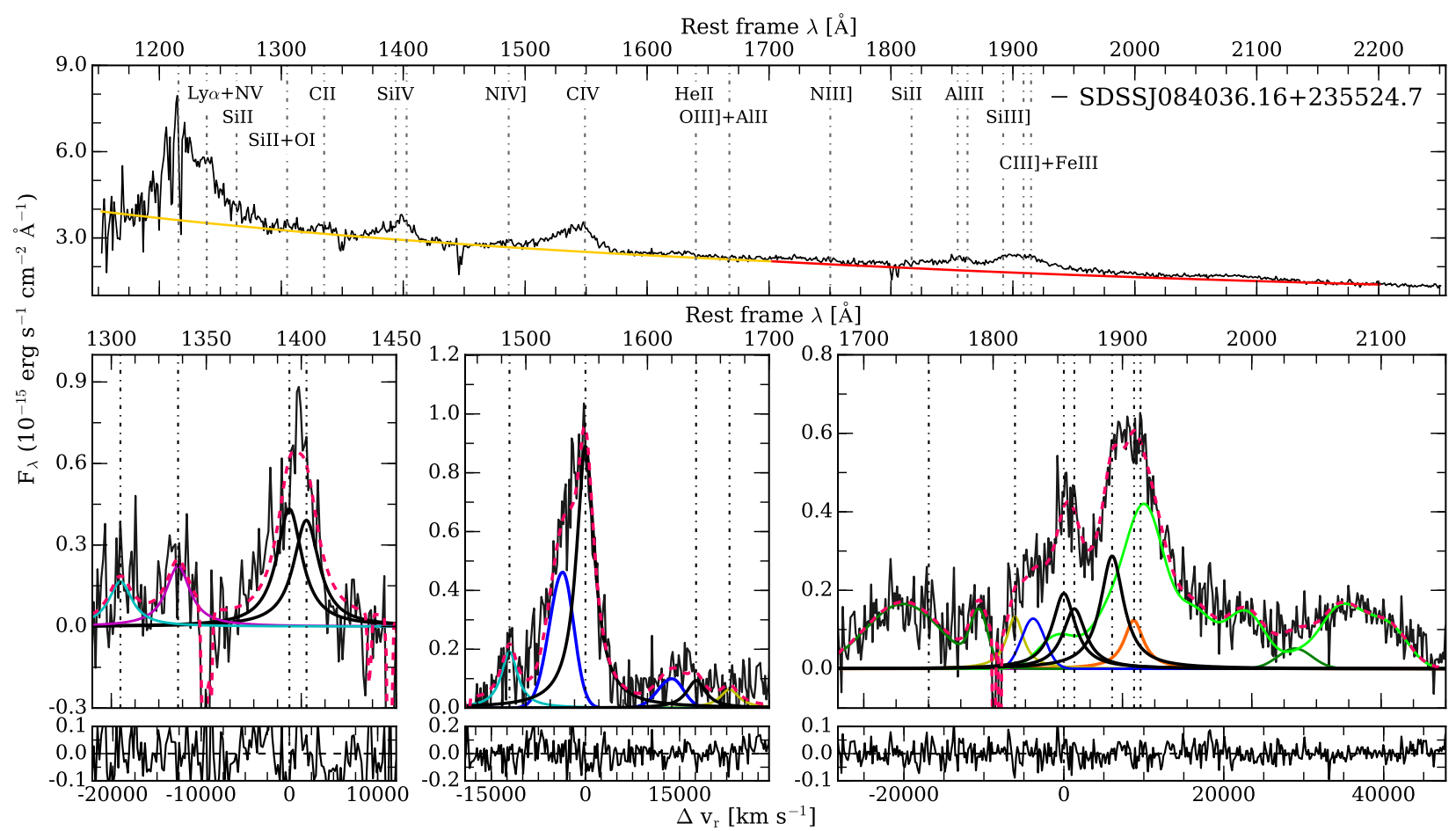

Fig. A.5. Same as Fig. A.1, for SDSSJ084036.16+235524.7.

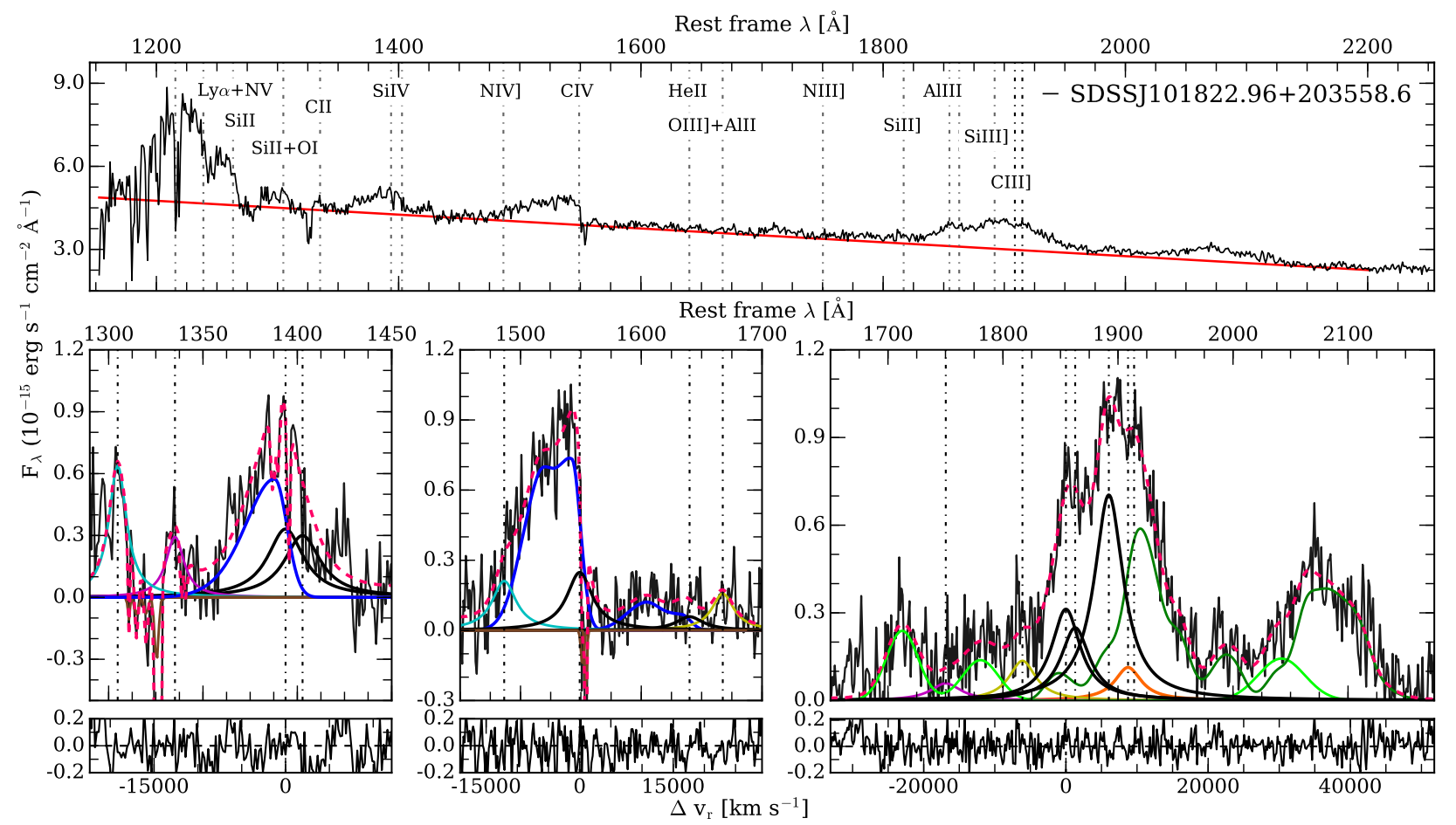

Fig. A.6. Same as Fig. A.1, for SDSSJ101822.96+203558.6. 


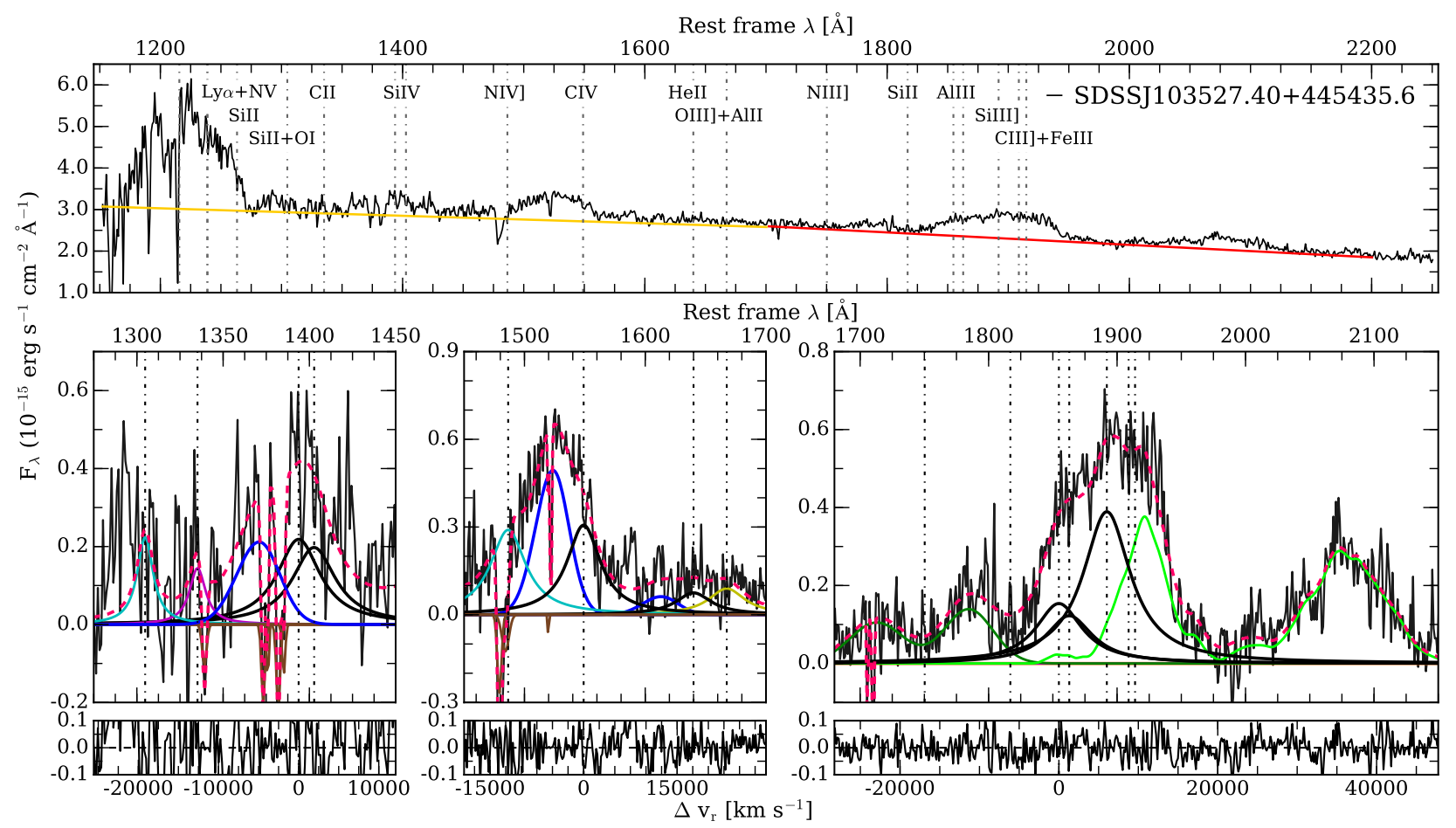

Fig. A.7. Same as Fig. A.1, for SDSSJ103527.40+445435.6.

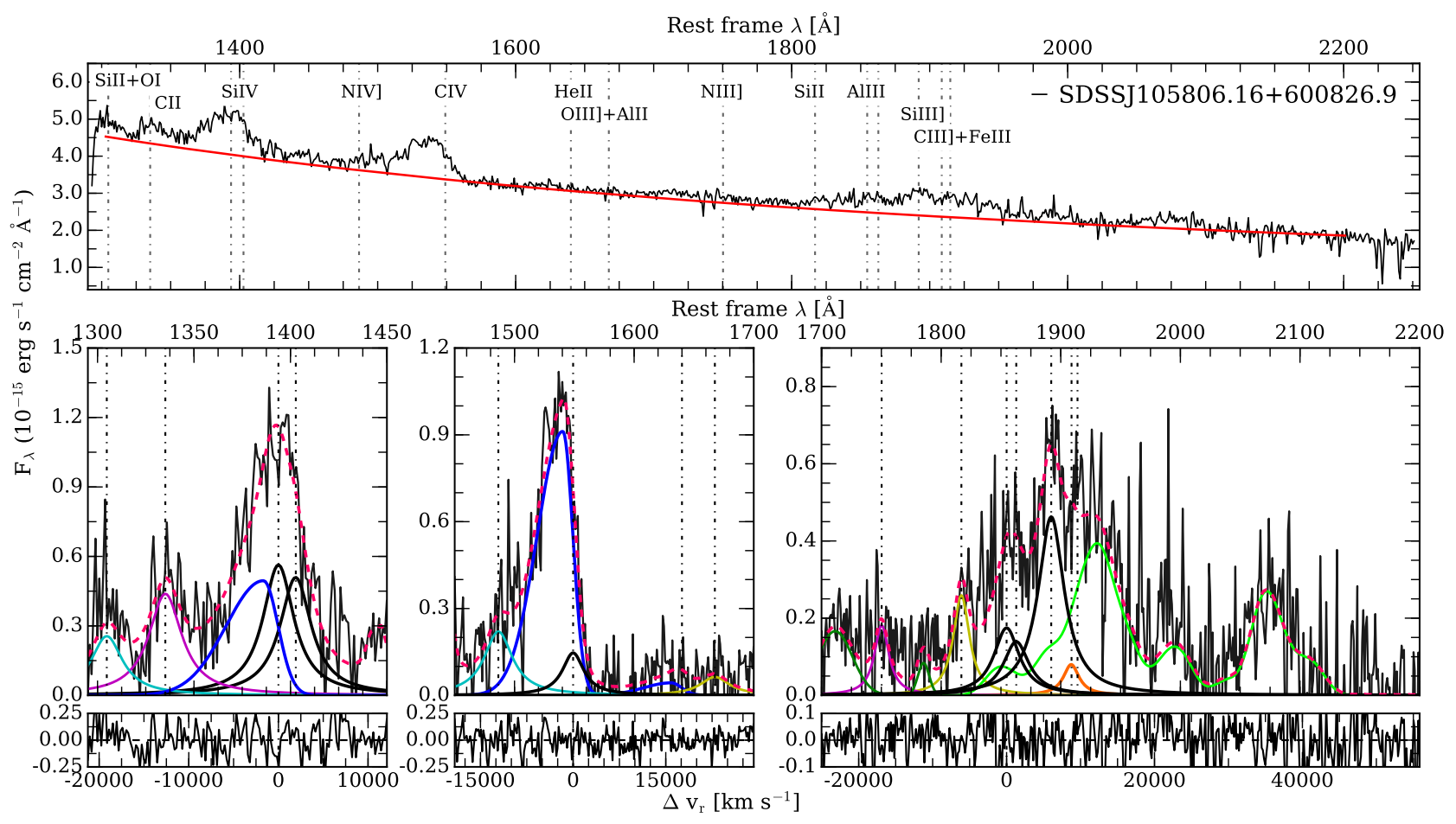

Fig. A.8. Same as Fig. A.1, for SDSSJ105806.16+600826.9. 
M. L. Martínez-Aldama et al.: Extreme quasars at high redshift

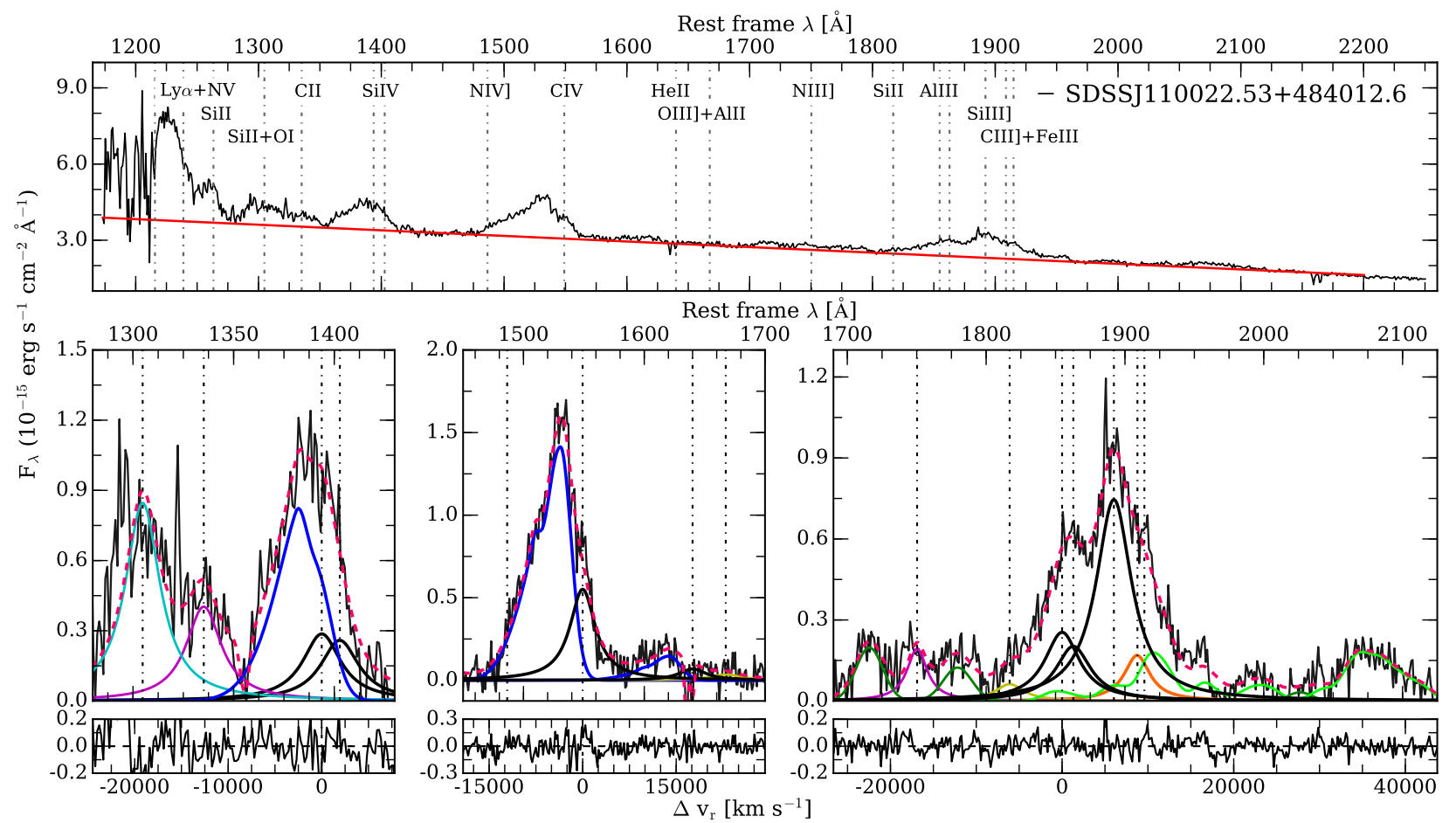

Fig. A.9. Same as Fig. A.1, for SDSSJ110022.53+484012.6.

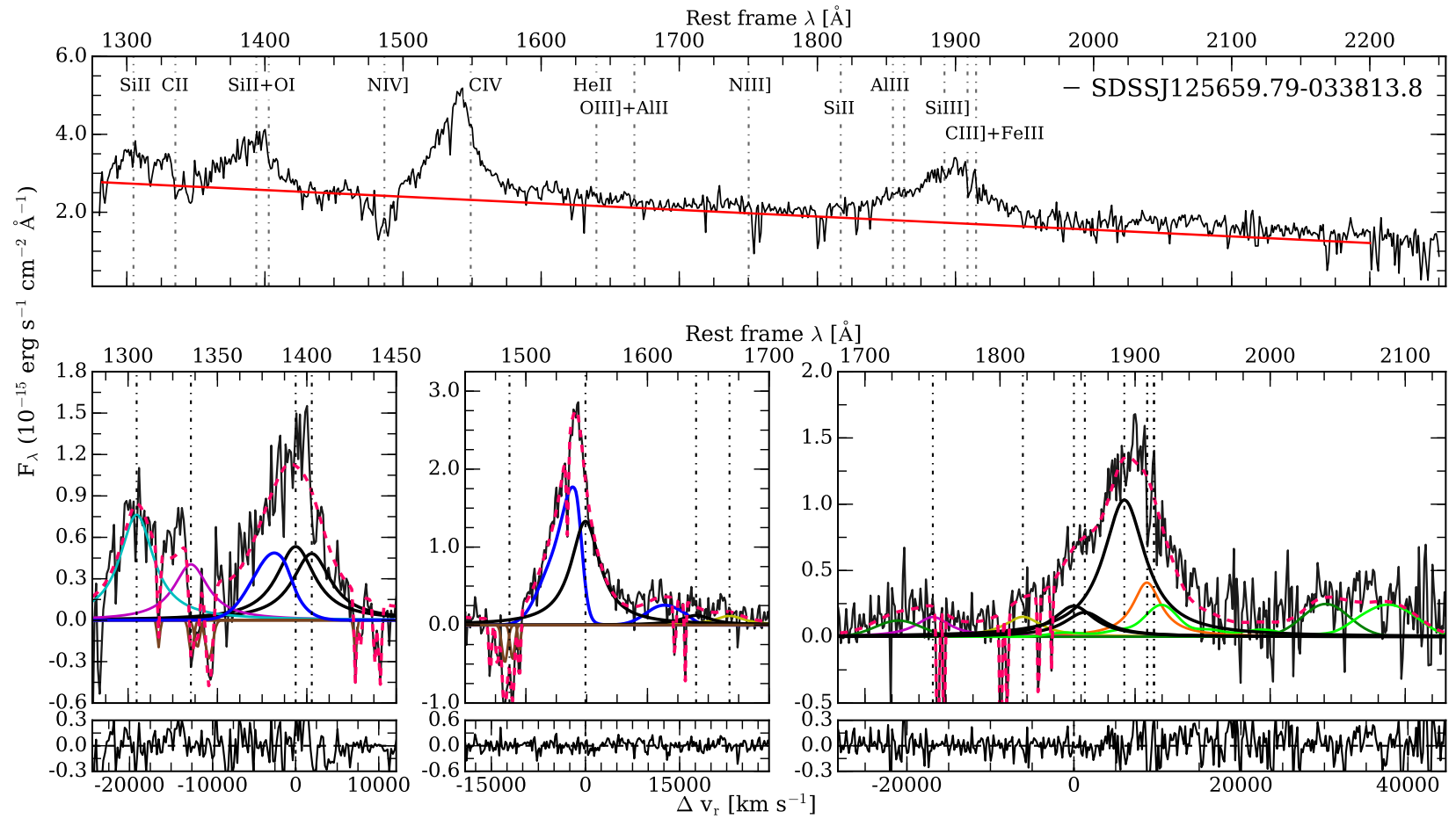

Fig. A.10. Same as Fig. A.1, for SDSSJ125659.79-033813.8. 


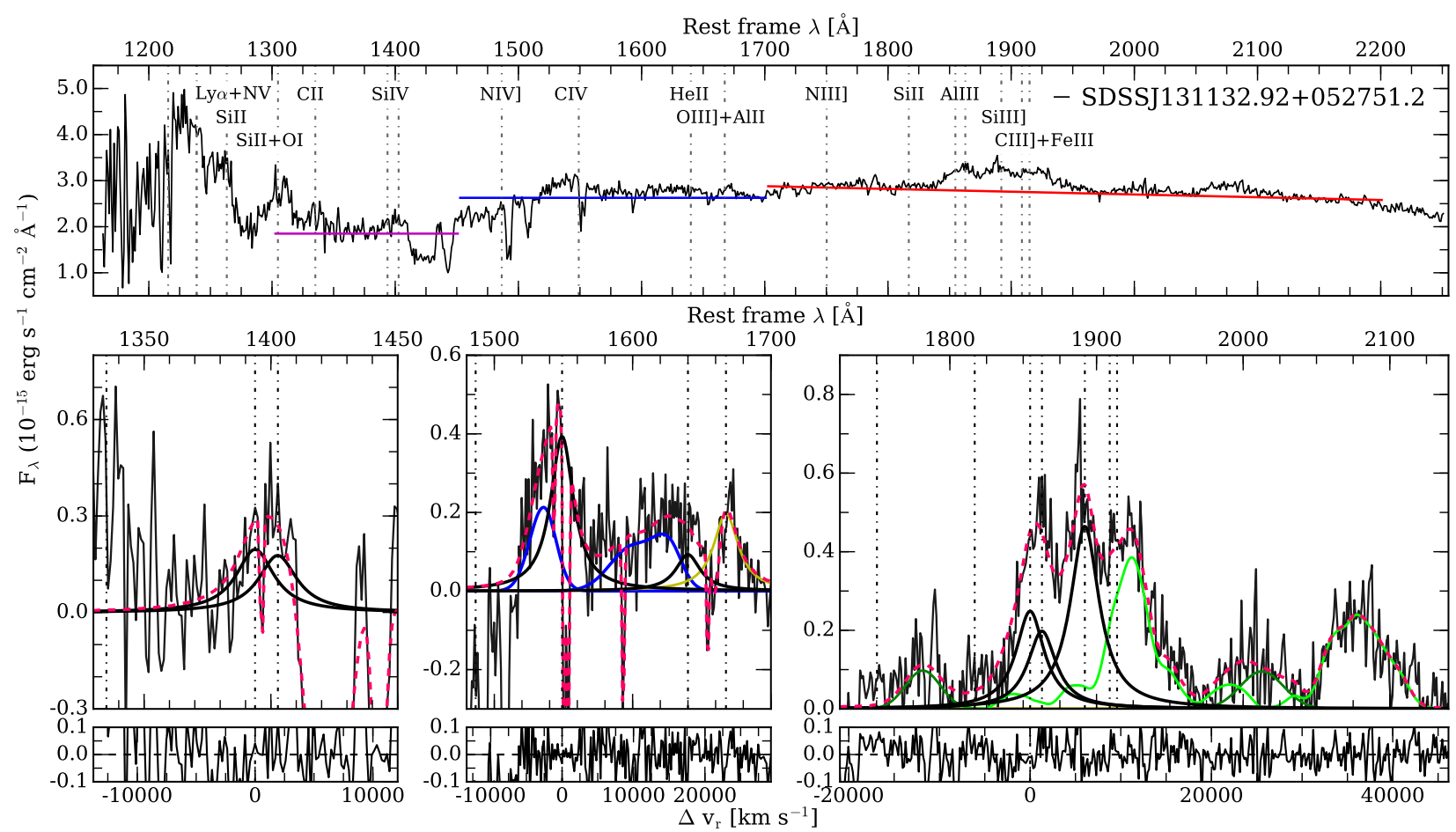

Fig. A.11. Same as Fig. A.1, for SDSSJ131132.92+052751.2.

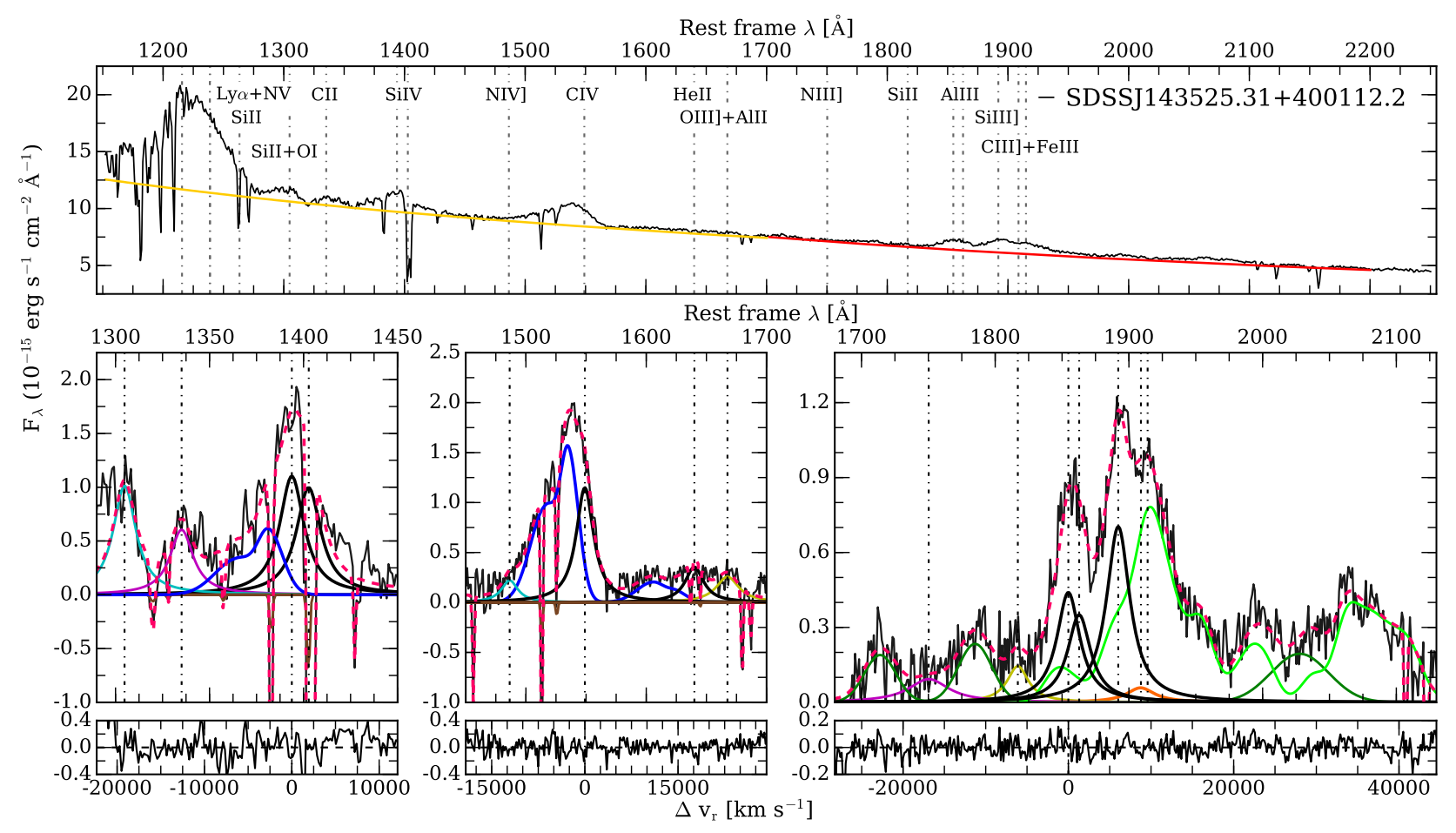

Fig. A.12. Same as Fig. A.1, for SDSSJ143525.31+400112.2. 
M. L. Martínez-Aldama et al.: Extreme quasars at high redshift

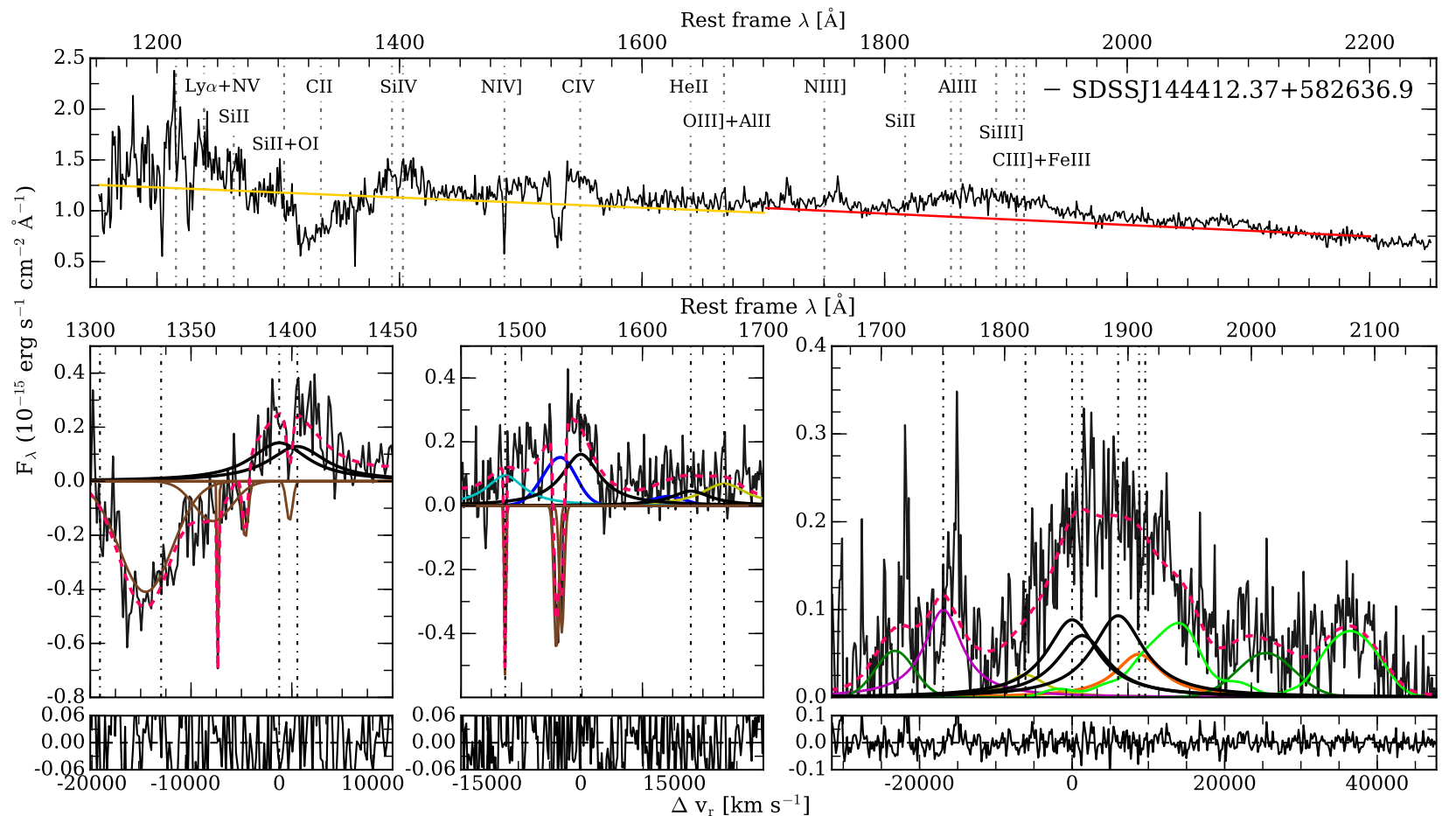

Fig. A.13. Same as Fig. A.1, for SDSSJ144412.37+582636.9.

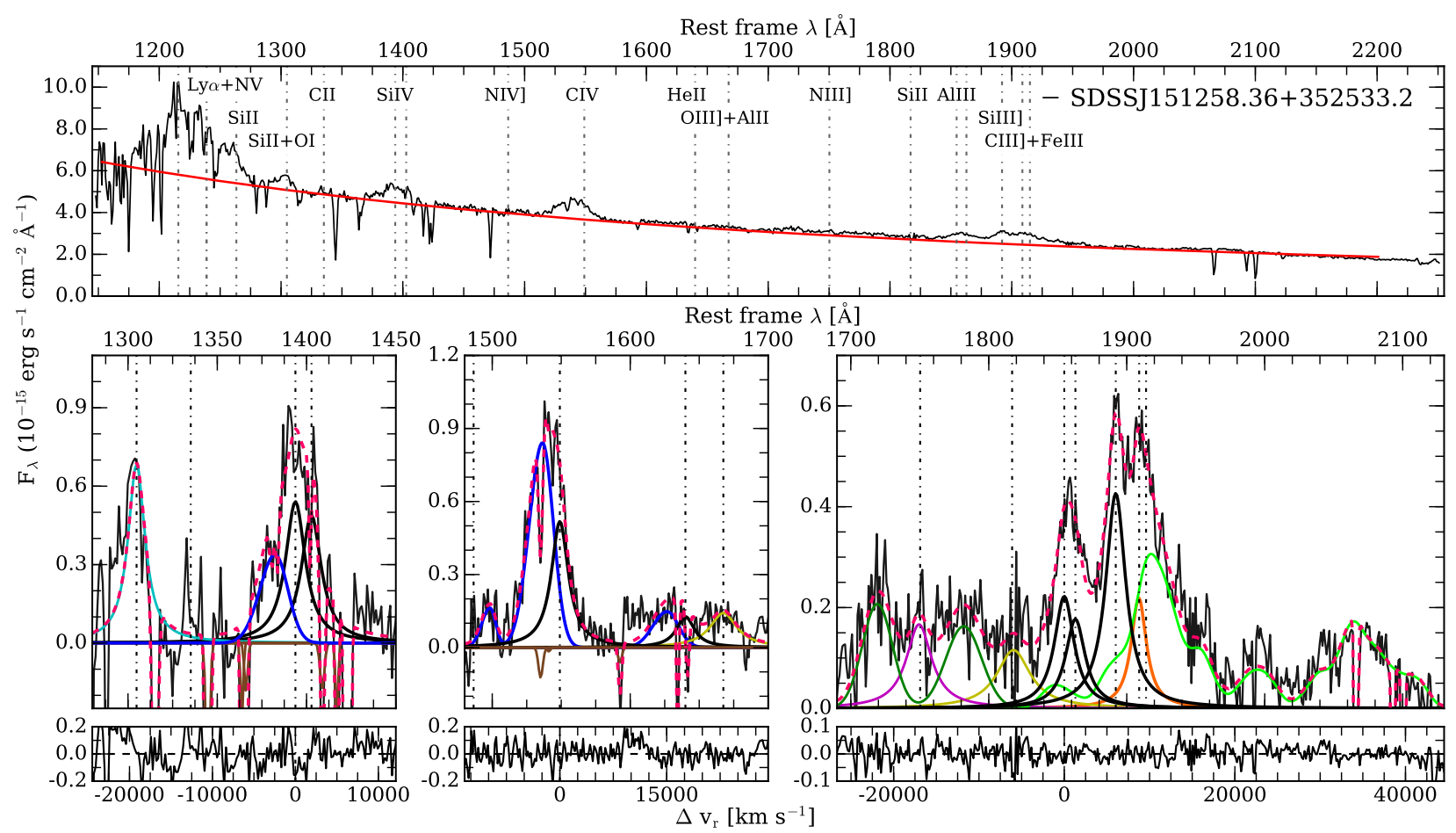

Fig. A.14. Same as Fig. A.1, for SDSSJ151258.36+352533.2. 


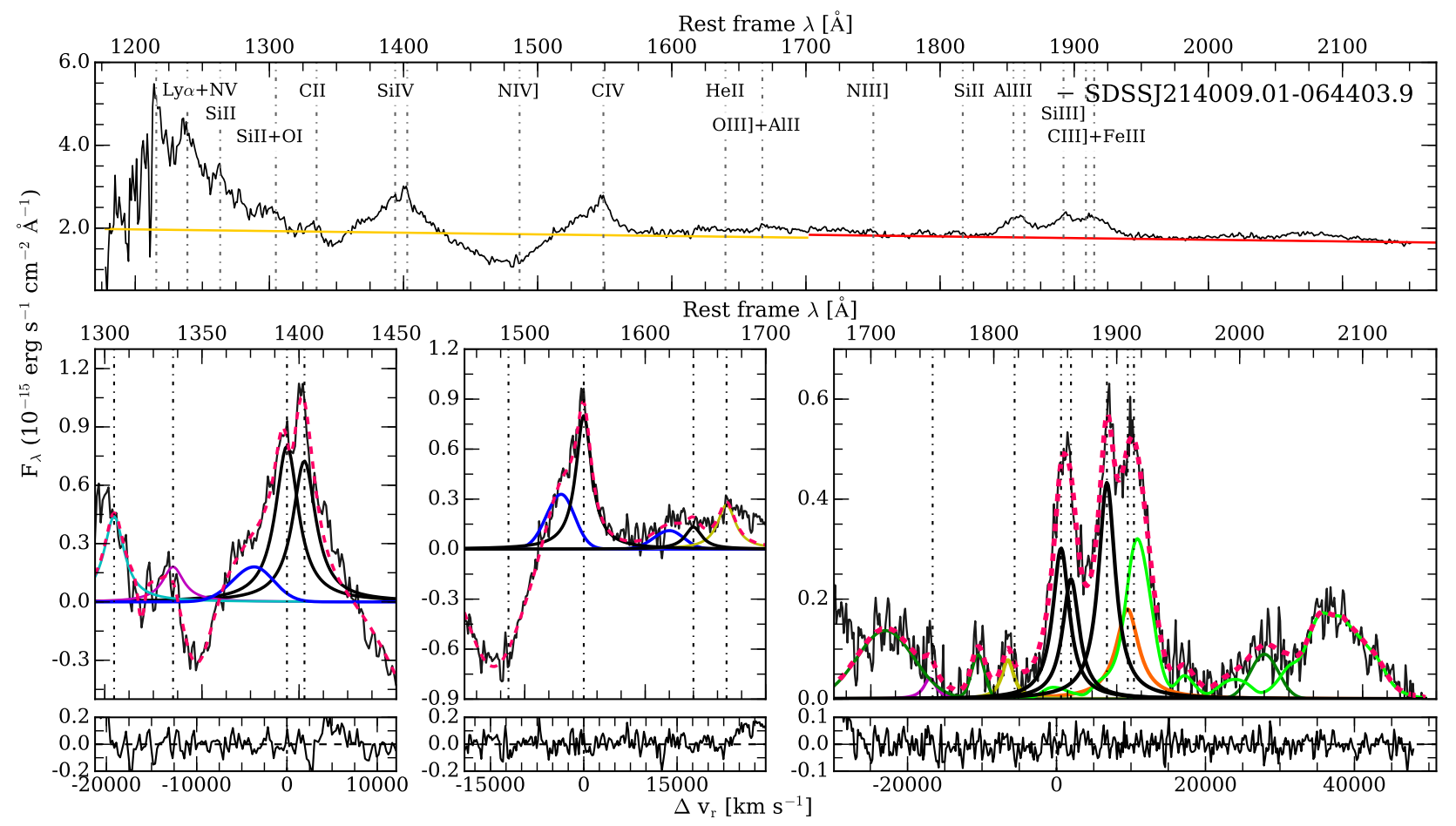

Fig. A.15. Same as Fig. A.1, for SDSSJ214009.01-064403.9.

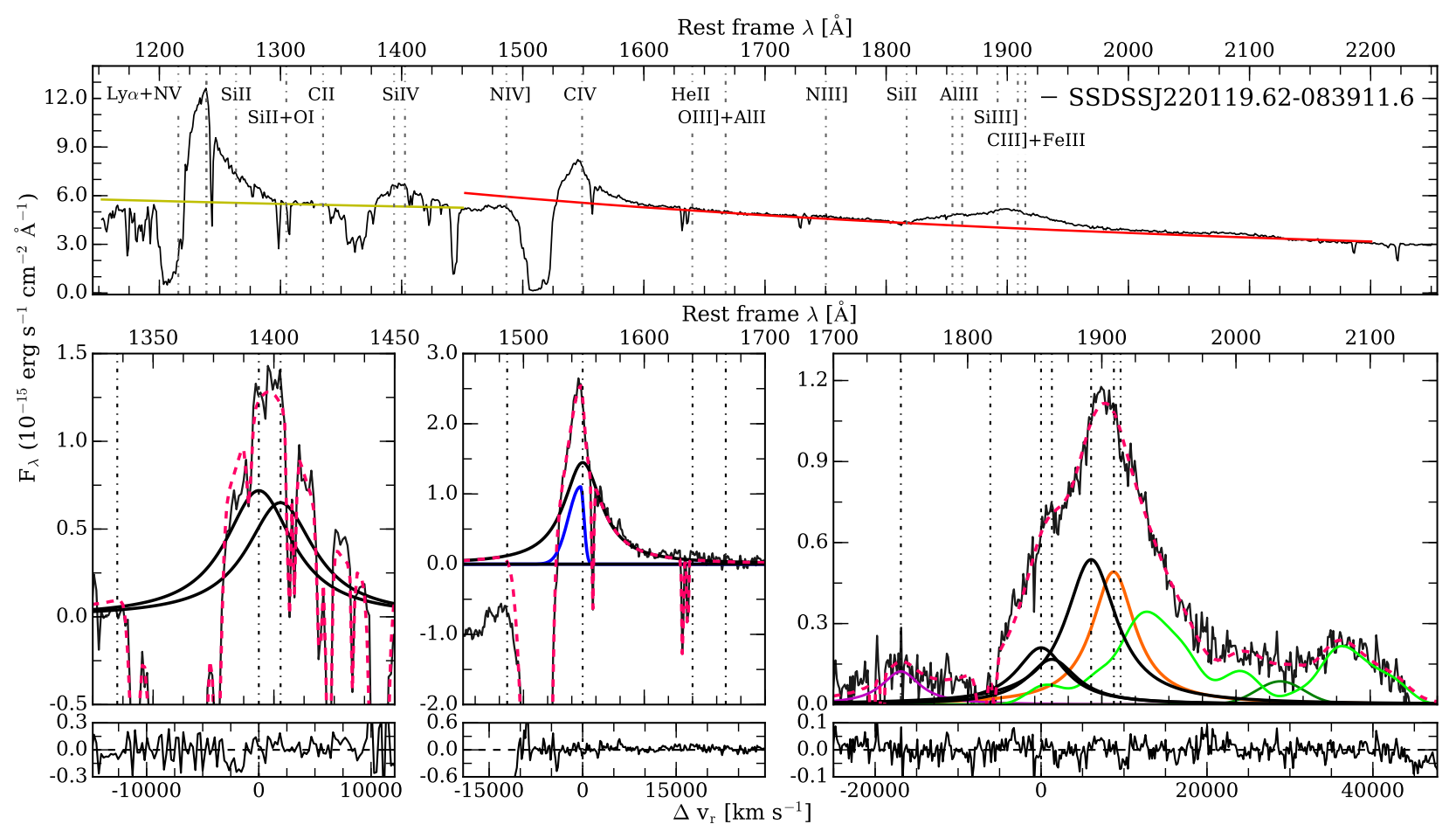

Fig. A.16. Same as Fig. A.1, for SDSSJ220119.62-083911.6. 
M. L. Martínez-Aldama et al.: Extreme quasars at high redshift

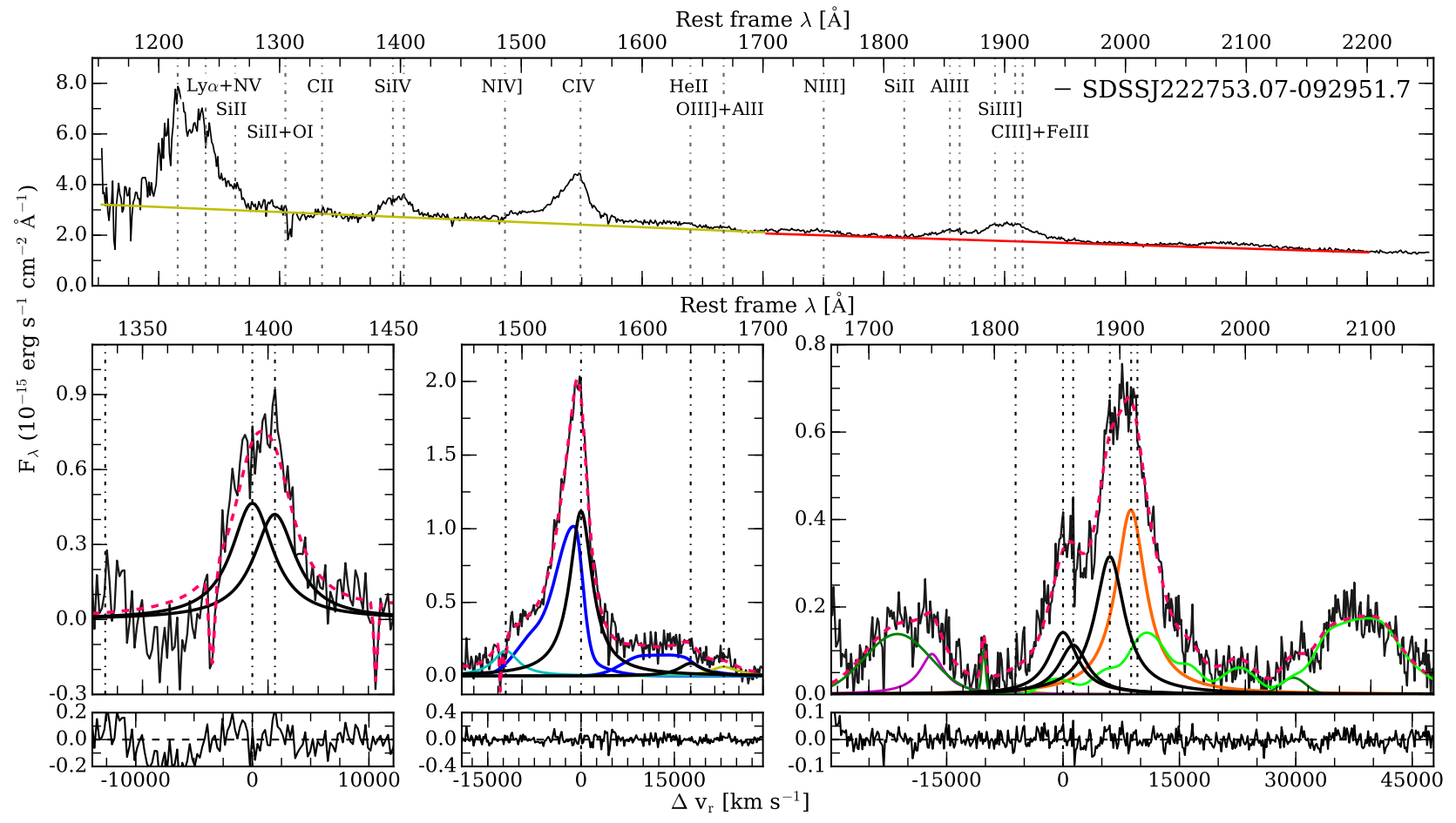

Fig. A.17. Same as Fig. A.1, for SDSSJ222753.07-092951.7.

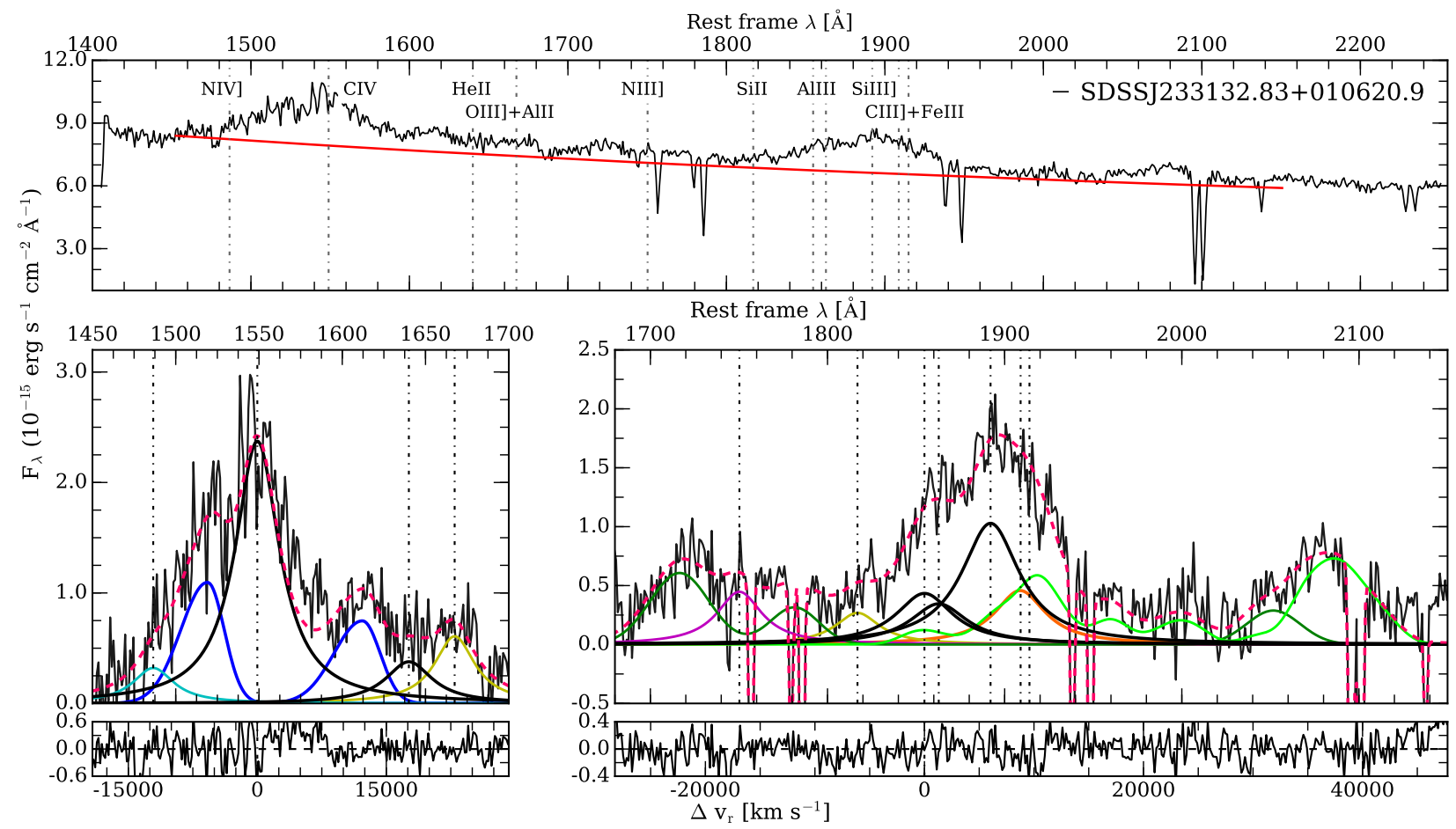

Fig. A.18. Same as Fig. A.1, for SDSSJ233132.83+010620.9. 


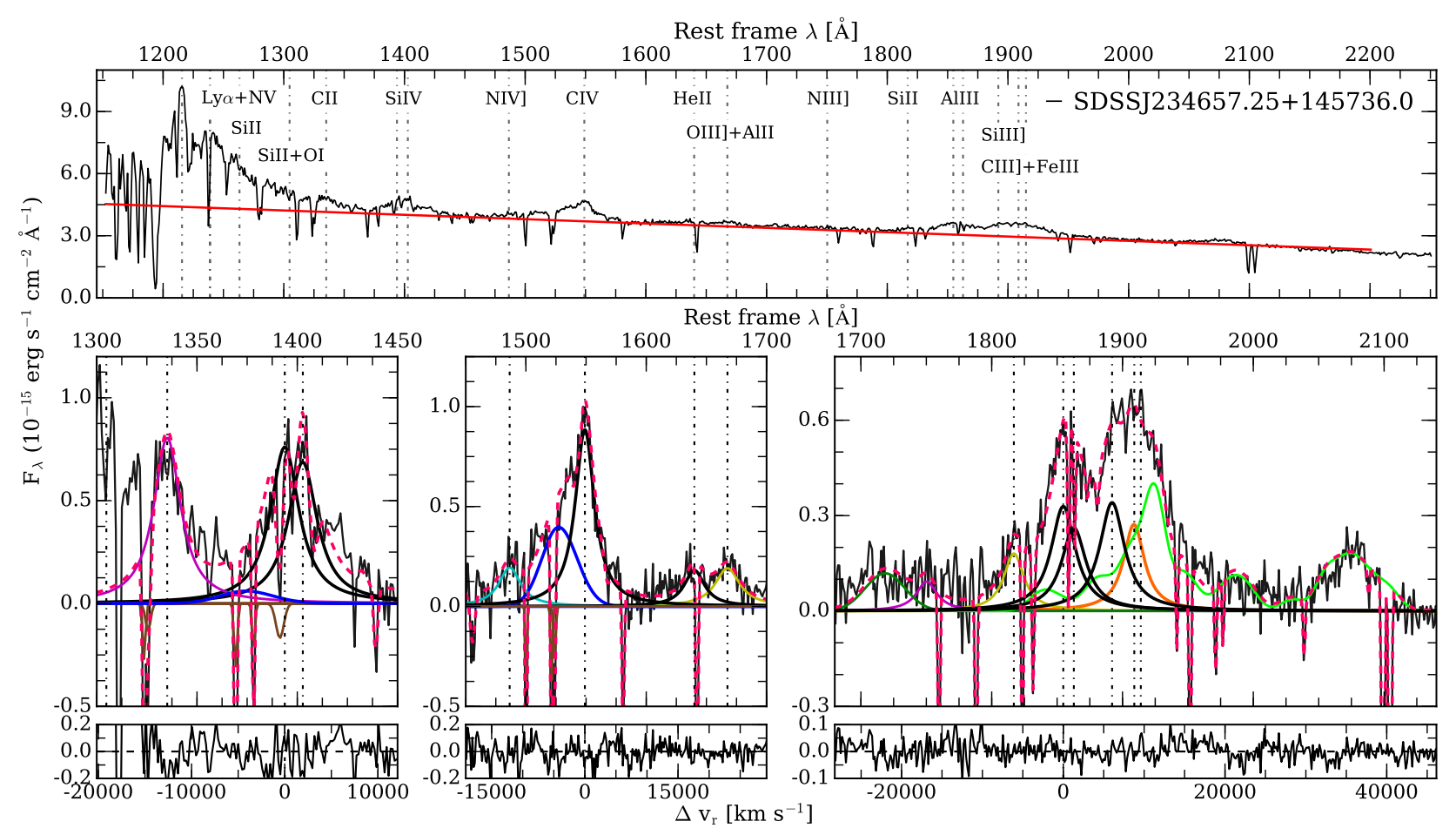

Fig. A.19. Same as Fig. A.1, for SDSSJ234657.25+145736.0.

\section{Appendix B: Error estimates}

Emission lines of the $\mathrm{xA}$ spectra are blended in almost all cases. For example, in the $1900 \AA$ blend the emission of CIII] $\lambda 1909$ and FeIII $\lambda 1914$ cannot be deblended; or in the case of CIV the separation between broad and blue components is not obvious.We built Monte Carlo (MC) simulations to determine the error estimates, in order to consider the effects of blending. The MC method allows us to consider all emission components and include a variation of the flux and FWHM simultaneously. The method is based on minimizing the $\chi^{2}$ (Barlow 1989). A variation in parameters of the line properties causes changes in the $\chi^{2}$. Then, the parameters at $1 \sigma$ confidence level are those that satisfy the constraint: $\chi_{1 \sigma}^{2} \leq \chi_{\min }^{2}+1$ (Andrae 2010).

The MC method is applied to the same spectral regions identified for the multicomponent fits with SPECFIT (Sect. 4.2). All line components considered in modeling the observed quasar emission spectrum were taken into account. For example, in the $1900 \AA$ blend region variations in flux and FWHM of AlIII, SiIII], CIII], SiII $\lambda 1816$, NIII] $\lambda 1750$, FeII and FeIII are considered for each simulation. Parameters vary around the values given by the SPECFIT model without any constraint and the simulations are therefore totally independent of the SPECFIT model.

We ran 5000 simulations for each quasar in order to obtain good sampling. Left panels of Fig. B. 1 show the distributions of $\chi^{2}$ for the flux of AlIII (top) and for the total CIV profile (bottom), for the quasar SDSSJ222753.07-092951.7. Usually 1000-3000 simulations satisfy the criterion $\chi_{1 \sigma}^{2} \leq \chi_{\min }^{2}+1$. The total width of the distribution at $\chi_{\min }^{2}+1$ is the $1 \sigma$ error confidence level we consider for each parameter. The central panels of Fig. B.1 show the $1900 \AA$ Alend (top) and CIV (bottom) spectra, and the quasar emission associated with the $1 \sigma$ confidence level derived from the MC simulations (gray shadow regions).

Strongest emission lines like AlIII or the total emission of CIV show Gaussian distributions for the flux and FWHM MC replications (see right panels of Fig. B.1). However, for weak lines like CIII] $\lambda 1909$, NIII] $\lambda 1750$ or HeII $\lambda 1640_{\mathrm{BC}}$, the distribution has an asymmetric or flat behavior. This is because faint lines are strongly affected by the presence of the stronger ones (e.g., SiII $\lambda 1816$ affected by AlIII ). In these cases, we consider the flux found by SPECFIT as an upper limit. We report the FWHM values followed by a double colon to signify a high uncertain value (Tables 4-7). 

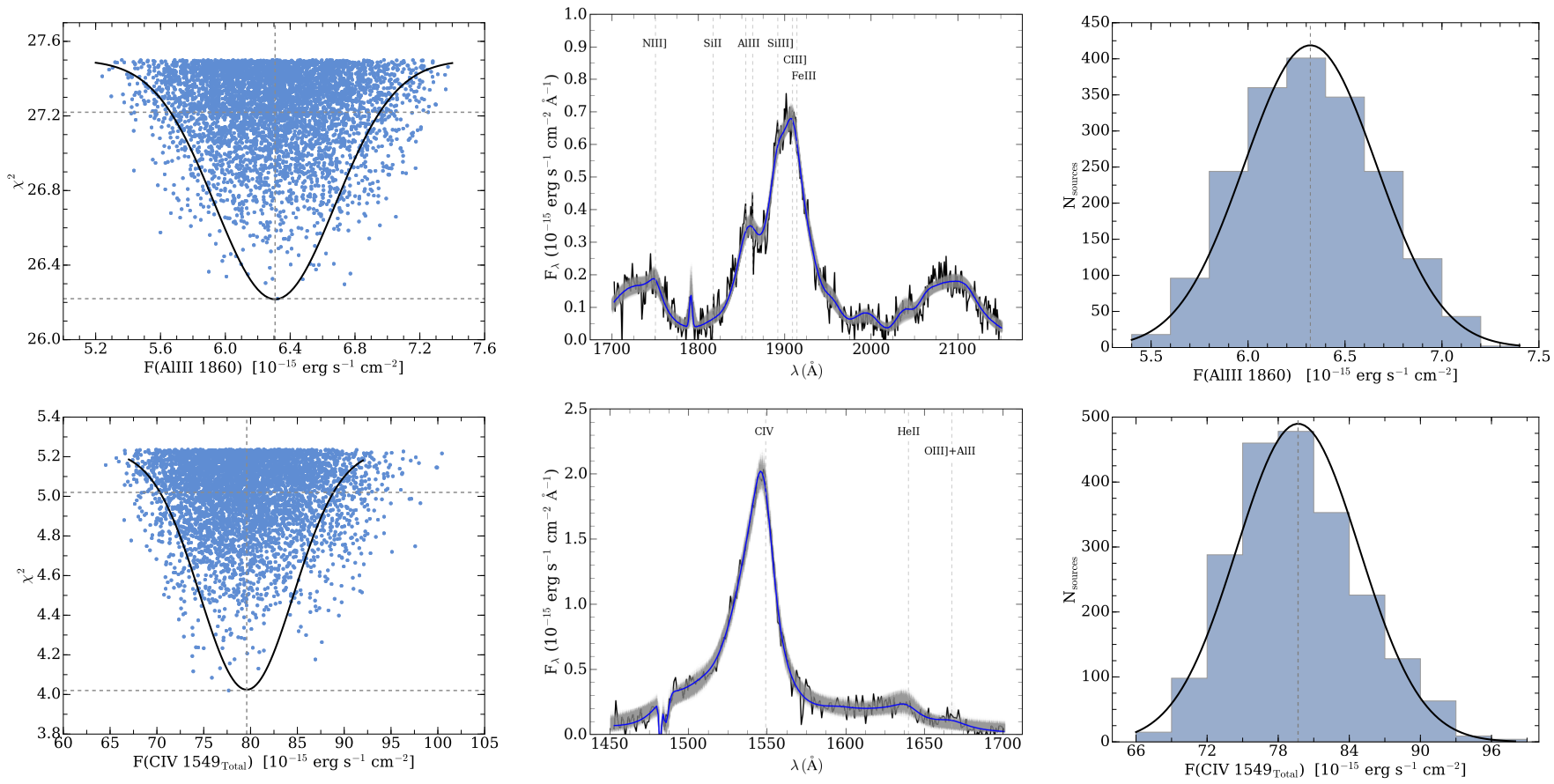

Fig. B.1. Left panels: distribution of $\chi^{2}$ as a function of the flux of AlIII (top) and total CIV profile (bottom). Black continuous lines mark the normal distribution associated with simulations. Gray horizontal lines indicate the value at $\chi_{\text {min }}^{2}$ and at $\chi_{\min }^{2}+1$. The gray vertical line indicates the mean of the normal distribution. Middle panels: spectra of the quasar SDSSJ222753.07-092951.7 in the $1900 \AA$ Alend (top) and total CIV $\lambda 1549$ (bottom) spectral ranges. Gray shadow regions correspond to the models at the $\chi_{\min }^{2}+1$ confidence level obtained from the Monte Carlo simulations. The blue line corresponds to the SPECFIT result. Right panels show the distributions of the fluxes of AlIII (top) and total CIV (bottom) for all the models under the $1 \sigma$ confidence level plotted in the left panels. Vertical lines has the same meaning as in left panels.

\section{Appendix C: Fell and Fell}

\section{C.1. Identification of Fell and Felll transitions}

In next lines are described the most important FeII and FeIII transitions in the spectral range $1700-2100 \AA$. Table C. 1 reports the approximate central wavelength of the FeII and FeIII features, the multiplet identification, and the sources where they are prominent.

\section{FeII}

[ $\lambda 1715 \AA]$ - This feature has not been identified previously as FeII emission. Graham et al. (1996) suggest AlII associated with the $3 p^{3} P^{o}-3 d^{3} D$ transitions. However, the wavelength consistency is poor, the AlII transition is fairly high level, and its intensity is predicted negligible even for very low ionization parameter $\left(\sim 10^{-3}\right)$. We therefore ascribe the feature to FeII UV multiplet \#38 (associated with the transition between terms $a^{4} F-{ }^{4} D^{o}$ ), and specifically to the transition ${ }^{4} D_{\frac{9}{2}}^{o} \rightarrow{ }^{4} F_{\frac{7}{2}}^{o}$ that has the higher oscillator strength among all FeII lines listed by Kurucz \& Bell (1995, $\log g f \approx-0.395)$.

[ $\lambda 1785 \AA]$ - The UV multiplet \#191 is strong in several quasars, most notably I Zw 1 (Marziani et al. 1996; Laor et al. 1997; Vestergaard \& Wilkes 2001). The emission is believed to be enhanced by a Ly $\alpha$ fluorescence mechanism (Baldwin et al. 2004) also operating in symbiotic stars and in stars with chromospheric activity (Johansson \& Hansen 1988; Johansson et al. 1995).

$[\lambda 2020 \AA]$ - This feature is never strong $(\lesssim 2 \AA)$, but is apparently detected in a few objects (Table C.1). The main feature could be associated with the line at $2020.739 \AA$ and other two lines of FeII multiplet UV \#83.

\section{FeIII}

[ $\lambda 1914 \AA]$ - The line of the FeIII UV multiplet \#34 is included in the template. We added a component to take into account the possibility of extraordinary enhancement due to Ly $\alpha$ fluorescence, as outlined in Marziani et al. (2010).

[ $\lambda 2005 \AA \& \lambda 2045 \AA$ ] - These features have been identified as due to FeIII UV \#55 (Graham et al. 1996; Vestergaard \& Wilkes 2001).

$[\lambda 2080 \AA]$ - This feature is perhaps the most prominent one among the FeIII features. Its identification FeIII UV \#48 is supported by the consistency in wavelength of the three multiplet components. The peculiarity of the GTC-xA sources is that the feature is not reproduced well by the template: an additional component is needed. The analysis of the $2080 \AA$ feature is reported in Sect. 6.4, and its origin is discussed in Appendix C.2 below.

[ $\lambda 2093 \AA]$ - We identify this feature as due to FeIII UV \#77 (Vestergaard \& Wilkes 2001). A previous identification as FeI seems less likely, considering that FeI features are predicted to be exceedingly weak (Sigut et al. 2004) even at the lowest ionization degrees.

[ $\lambda 2115 \AA]$ - Vestergaard \& Wilkes (2001) associates this feature with FeIII UV \#58.

\section{C.2. On the origin of Felll emission}

The strength of FeIII emission is surprising, given that the overall appearance of the spectrum is suggestive of extremely low ionization. The FeIII features have also been observed in Pop. A sources or associated with extreme Pop. A (e.g., Baldwin et al. 1996; Graham et al. 1996; Richards et al. 2011). The FeIII lines 


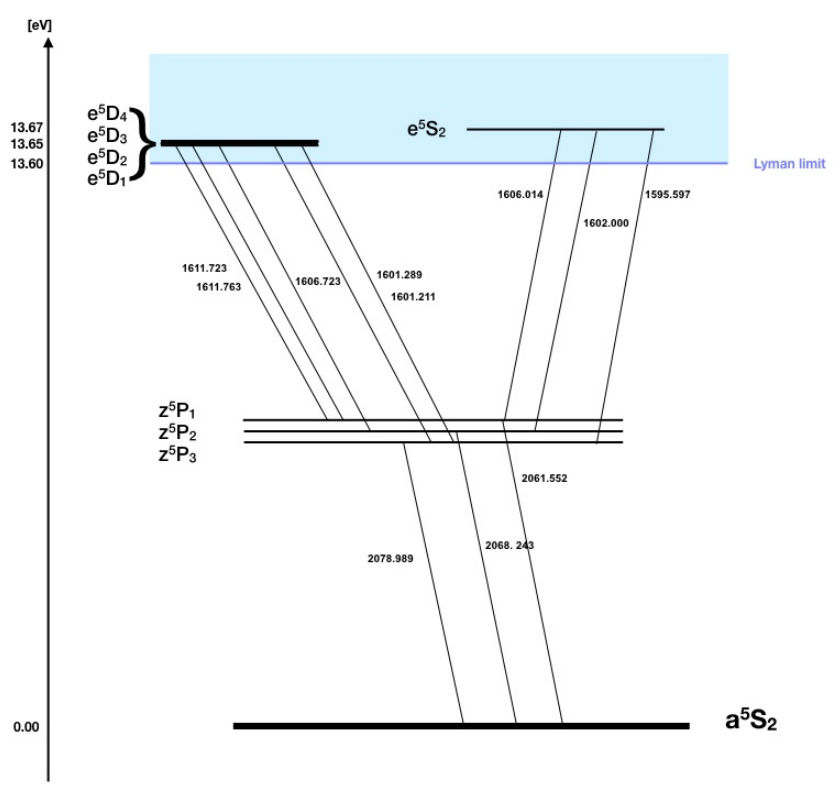

Fig. C.1. Partial Grotrian diagram showing the energy levels and the spectroscopic terms associated with the emission of FeIII multiplet \#48, \#118 (top left) and \#119 (top right). Energy level separation is not drawn to scale for clarity.

Table C.1. Identification of FeII and FeIII features.

\begin{tabular}{ccc}
\hline \hline $\begin{array}{c}\text { Feature } \\
(\AA)\end{array}$ & Identification & Sources where is detected \\
\hline$\lambda 1715$ & FeII UV \#38 & J000807, J021606, J084036, J214009, J222753 \\
$\lambda 1785$ & FeII UV \#191 & J125659, J101822, J144412 \\
$\lambda 2005$ & FeIII UV \#55 & J233132 \\
$\lambda 2020$ & FeII UV \#83 & J021606, J233132, J214009 \\
$\lambda 2045$ & FeIII UV \#60 & J222753, J110022, J151258 \\
$\lambda 2080$ & FeIII UV \#48 & J000807, J004241, J021606, J101822, J103527, \\
& & J125659, J214009, J222753 \\
$\lambda 2093$ & FeIII UV \#77 & J105806 \\
$\lambda 2115$ & FeIII UV \#58 & J220119, J222753, J110022 \\
\hline
\end{tabular}

are mainly emitted in a region at the boundary between the fully and partially ionized zone. At ionization parameter $\log U \gtrsim-2$, the region of FeIII line formation is usually a region where the opacity in the Lyman continuum grows to values $\tau_{912} \gtrsim 1$ (i.e., a small fraction of the geometric depth of the emitting gas of slab). Deeper into the slab from its illuminated face, the dominant ionization stage is $\mathrm{Fe}^{+3}$. A significant fraction of FeIII is present at the illuminated face of the cloud within the HII zone, but only if the ionization parameter is very $\operatorname{low}(\log U \lesssim-3$, Fig. 3 of Sigut et al. 2004). $\mathrm{Fe}^{+2}$ is $\lesssim 2 \%$ of $\mathrm{Fe}^{+1}$ in the partially-ionized zone (PIZ): $\mathrm{Fe}^{+1}$ still remains the dominant ionization stage of iron in the PIZ, as required by the strong optical FeII emission required for these sources. In the spectral range between 1800 and $2100 \AA$, however, the dominant contribution may be due to FeIII, as predicted by the models of Sigut et al. (2004), which assumes $\log U \sim-3$.

The role of Ly $\alpha$ fluorescence in explaining the UV and IR FeII emission has been known since the late 1980s from photoionization simulations (e.g., Sigut \& Pradhan 1998; Verner et al. 2004; Sigut et al. 2004) as well as from observational evidence (e.g., Penston 1987; Marinello et al. 2016; Clowes et al. 2016, and references therein). As mentioned, a specific feature that is believed to be enhanced by Ly $\alpha$ fluorescence is the FeII $\# 191$ multiplet at $1785 \AA$. Also the FeIII line at $\lambda 1914$, ascribed to the transition of the FeIIUV \#34 multiplet $\mathrm{z}^{7} P_{3}^{o} \rightarrow \mathrm{a}^{7} S_{3}$, is expected to be enhanced by Ly $\alpha$ fluorescence. Although this is not a resonant line (the lower level is $\sim 3 \mathrm{eV}$ above ground), the line appears often stronger than in the Vestergaard \& Wilkes (2001) template, presumably because the upper level is populated by $\operatorname{Ly} \alpha$ fluorescence (Marziani et al. 2010). Figure 8 suggest a close association between the 2080 feature and the FeII and FeIII features enhanced by Ly $\alpha$ fluorescence.

Generally speaking, we expect that $\operatorname{Ly} \alpha$ fluorescence may be also relevant in the explanation of the overall FeIII spectrum, considering the large number of radiative transitions possible for the electronic configuration of the $\mathrm{Fe}^{++}$. The FeIII high ionic fraction in the HII zone and at the transition zone between HII and PIZ are expected to be conditions that make the fluorescent absorption of Lyman continuum photons by FeIII ions especially efficient. In the specific case of the 2080 feature, we suggest that a mechanism of enhancement is due to fluorescence with the Lyman continuum ionization edge. The FeIII UV \#48 multiplet is radiatively linked to the multiplets \#118 and \#119 between the terms $z^{5} P^{0} \mathrm{e}^{5} D$ and $\mathrm{e}^{5} S$, respectively. The energy difference between the lower level of the lower a ${ }^{5} S$ term of multiplet \#48 and the upper terms of \#118 and 119 , is 13.65 and $13.67 \mathrm{eV}$, respectively, slightly above the ionization potential of hydrogen $(13.5984 \mathrm{eV})$. Figure C.1 schematically shows the energy levels associated with the spectroscopic terms. Even if the energy is not strictly coincident, the Lyman continuum emission (which is expected to decrease with $v^{-3}$ ) may act as a broadened pseudoline.

If this mechanism is indeed operating, we expect FeIII emission from multiplets UV \#118 and \#119 in the range 1595$1610 \AA$. A faint hump present on the composite spectrum in the predicted range could be due to some FeIII emission as well as to the blueshifted component of HeII $\lambda 1640$ blending with the red side of CIV 21549 . Considering the weakness of the features, and the severe blending with HeII $\lambda 1640$ and CIV $\lambda 1549$, the prediction of FeIII emission around $1600 \AA$ should be verified on high $\mathrm{S} / \mathrm{N}$ spectra of sources with narrower lines. 